\title{
Implementing the Quantum von Neumann Architecture with Superconducting Circuits
}

Matteo Mariantoni ${ }^{1,4, \S}, \mathrm{H}$. Wang ${ }^{1, *}$, T. Yamamoto $^{1,2}$, M. Neeley $^{1, \dagger}$, Radoslaw C. Bialczak ${ }^{1}$, Y. Chen ${ }^{1}$, M. Lenander ${ }^{1}$, Erik Lucero ${ }^{1}$, A. D. O'Connell ${ }^{1}$, D. Sank ${ }^{1}$, M. Weides ${ }^{1, \ddagger}$, J. Wenner $^{1}$, Y. Yin ${ }^{1}$, J. Zhao $^{1}$, A. N. Korotkov ${ }^{3}$, A. N. Cleland ${ }^{1,4}$, and John M. Martinis ${ }^{1,4, \S}$

${ }^{1}$ Department of Physics, University of California, Santa Barbara, CA 93106-9530, USA

${ }^{2}$ Green Innovation Research Laboratories, NEC Corporation, Tsukuba, Ibaraki 305-8501, Japan

${ }^{3}$ Department of Electrical Engineering, University of California, Riverside, CA 92521, USA ${ }^{4}$ California

NanoSystems Institute, University of California, Santa Barbara, CA 93106-9530, USA

*Present address: Department of Physics, Zhejiang University, Hangzhou 310027, China.

${ }^{\dagger}$ Present address: Lincoln Laboratory, Massachusetts Institute of Technology, 244 Wood Street,

Lexington, MA 02420-9108, USA.

${ }_{\ddagger}^{\ddagger}$ Present address: National Institute of Standards and Technology, Boulder, C0 80305, USA.

$\S$ To whom correspondence should be addressed. E-mail: matmar@physics.ucsb.edu (M. M.);

martinis@physics.ucsb.edu (J. M. M.)

last updated: September 20, 2011

The von Neumann architecture for a classical computer comprises a central processing unit and a memory holding instructions and data. We demonstrate a quantum central processing unit that exchanges data with a quantum random-access memory integrated on a chip, with instructions stored on a classical computer. We test our quantum machine by executing codes that involve seven quantum elements: Two superconducting qubits coupled through a quantum bus, two quantum memories, and two zeroing registers. Two vital algorithms for quantum computing are demonstrated, the quantum Fourier transform, with $66 \%$ process fidelity, and the three-qubit Toffoli OR phase gate, with $98 \%$ phase fidelity. Our results, in combination especially with longer qubit coherence, illustrate a potentially viable approach to factoring numbers and implementing simple quantum error correction codes.

Quantum processors ${ }^{1+4}$ based on nuclear magnetic resonance ${ }^{5+7}$, trapped ions ${ }^{8-10}$, and semiconducting devices ${ }^{11}$ were used to realize Shor's quantum factoring algorithm ${ }^{5]}$ and quantum error correction ${ }^{6 / 8}$. The quantum operations underlying these algorithms include two-qubit gates $\frac{223}{2}$, the quantum Fourier transform ${ }^{7 / 9}$, and threequbit Toffoli gates $\frac{10 \mid 12}{}$. In addition to a quantum processor, a second critical element for a quantum machine is a quantum memory, which has been demonstrated, e.g., using optical systems to map photonic entanglement into and out of atomic ensembles 13 .

Superconducting quantum circuits ${ }^{14}$ have met a number of milestones, including demonstrations of two-qubit gates $\sqrt{5 / 15}-17[19 \sqrt[20]{ }$ and the advanced control of both qubit and photonic quantum states $7 \sqrt{19 \sqrt[20]{22}}$. We demonstrate a superconducting integrated circuit that combines a processor, executing the quantum Fourier transform and a three-qubit Toffoli-class OR gate, with a memory and a zeroing register in a single device. This combination of a quantum central processing unit (quCPU) and a quantum random-access memory (quRAM), which comprise two key elements of a classical von Neumann architecture, defines our quantum von Neumann architecture.

In our architecture (Fig. 1A), the quCPU performs one-, two-, and three-qubit gates that process quantum information, and the adjacent quRAM allows quantum information to be written, read out, and zeroed. The quCPU includes two superconducting phase qubits $\frac{5 / 7 / 19[22]}{\mathrm{Q}_{1}}$ and $\mathrm{Q}_{2}$, connected through a coupling bus provided 

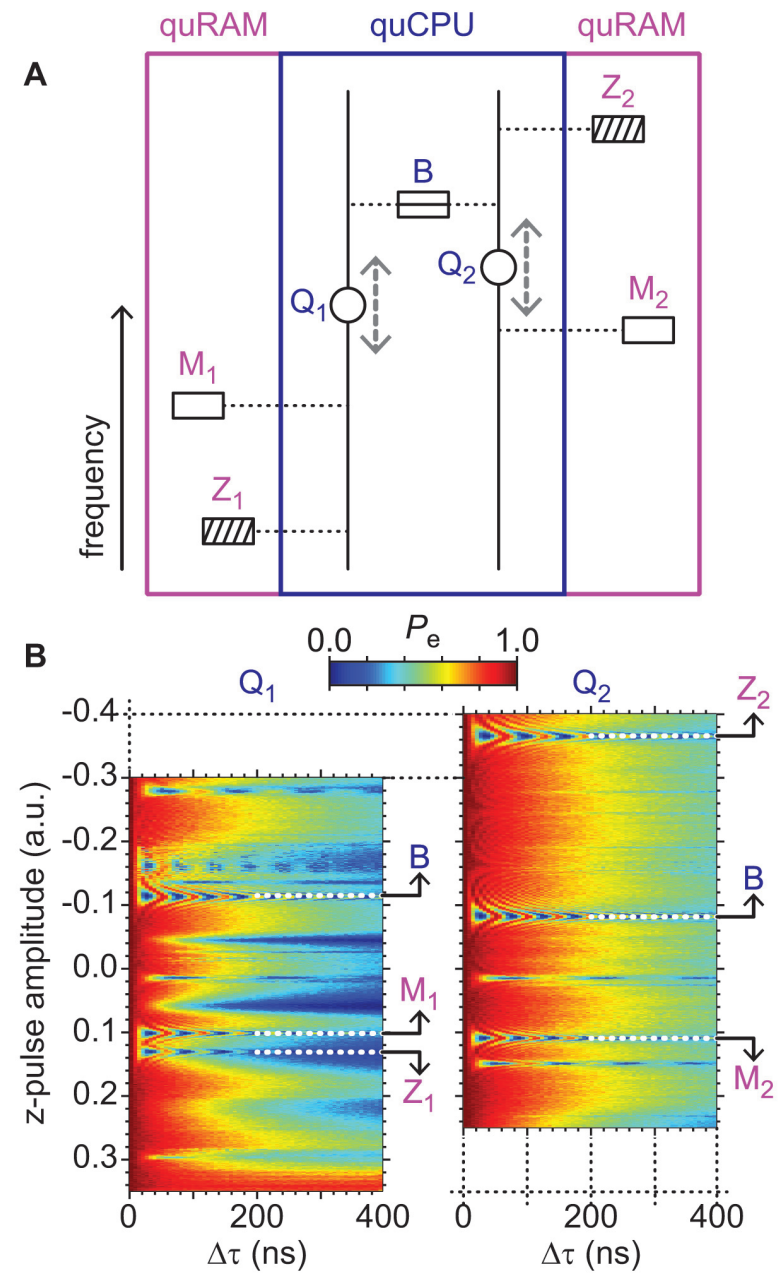

Figure 1: The quantum von Neumann architecture. (A) The quCPU (blue box) includes two qubits $Q_{1}$ and $Q_{2}$ and the bus resonator $B$. The quRAM (magenta boxes) comprises two memories $M_{1}$ and $M_{2}$ and two zeroing registers $Z_{1}$ and $Z_{2}$. The horizontal dotted lines indicate connections between computational elements. The vertical direction represents frequency, where the memory and zeroing registers are fixed in frequency, while the qubit transition frequencies can be tuned via z-pulses (grey dashed double arrows). (B) Swap spectroscopy ${ }^{[7}$ for $Q_{1}$ (left) and $\mathrm{Q}_{2}$ (right): Qubit excited state $|\mathrm{e}\rangle$ probability $P_{\mathrm{e}}$ (color scale) vs. z-pulse amplitude (vertical axis) and delay time $\Delta \tau$ (horizontal axis), after exciting the qubit with a $\pi$-pulse. At zero z-pulse amplitude the qubits are at their idle points, where they have an energy relaxation time $T_{\text {rel }} \simeq 400 \mathrm{~ns}$. A separate Ramsey experiment yields the qubits' dephasing time $T_{\text {deph }} \simeq 200 \mathrm{~ns}$. By tuning the z-pulse amplitude, the qubit transition frequencies $f_{\mathrm{Q}_{1}}$ and $f_{\mathrm{Q}_{2}}$ can be varied between $\simeq 5.5$ and $8 \mathrm{GHz}$. For z-pulse amplitudes indicated by $\mathrm{B}$ and $\mathrm{M}_{1}$ for $\mathrm{Q}_{1}$, and by $B$ and $M_{2}$ for $Q_{2}$, the "chevron pattern" of a qubit-resonator interaction is observed 7 . The transition frequencies of $B, M_{1}$, and $M_{2}$ are $f_{\mathrm{B}}=6.82 \mathrm{GHz}, f_{\mathrm{M}_{1}}=6.29 \mathrm{GHz}$, and $f_{\mathrm{M}_{2}}=6.34 \mathrm{GHz}$, respectively. From the chevron oscillation we obtain the qubit-resonator coupling strengths, which for both the resonator bus and the memories are $\simeq 20 \mathrm{MHz}$ (splitting) for the $|\mathrm{g}\rangle \leftrightarrow|\mathrm{e}\rangle$ qubit transition, and $\approx \sqrt{2}$ faster for the $|\mathrm{e}\rangle \leftrightarrow|\mathrm{f}\rangle$ transition $\left(|\mathrm{g}\rangle,|\mathrm{e}\rangle\right.$, and $|\mathrm{f}\rangle$ are the three lowest qubit states) ${ }^{22}$. For all resonators $T_{\text {rel }} \simeq 4 \mu \mathrm{s}$. Swap spectroscopy also reveals that the qubits interact with several modes associated with spurious two-level systems. Two of them, $Z_{1}$ and $Z_{2}$, are used as zeroing registers. Their transition frequencies are $f_{\mathrm{Z}_{1}}=6.08 \mathrm{GHz}$ and $f_{\mathrm{Z}_{2}}=7.51 \mathrm{GHz}$, respectively, with coupling strength to the qubits of $\simeq 17 \mathrm{MHz}$.

by a superconducting microwave resonator $\mathrm{B}$. The quRAM comprises two superconducting resonators $\mathrm{M}_{1}$ and $\mathrm{M}_{2}$ that serve as quantum memories, as well as a pair of zeroing registers $\mathrm{Z}_{1}$ and $\mathrm{Z}_{2}$, two-level systems that are used to dump quantum information. The chip geometry is similar to that in Refs. ${ }^{722}$, with the addition of the two zeroing registers. Figure 1B shows the characterization of the device by means of swap spectroscopy ${ }^{7}$.

The computational capability of our architecture is displayed in Fig. 2A, where a 7-channel quantum circuit, yielding a 128 dimensional Hilbert space, executes a prototypical algorithm. First, we create a Bell state between $\mathrm{Q}_{1}$ and $\mathrm{Q}_{2}$ using a series of $\pi$-pulse, $\sqrt{\text { iSWAP, }}$ and iSWAP operations (step I, a to c) ${ }^{22}$. The corresponding density matrix $\hat{\rho}_{(\mathrm{I})}[\mathrm{Fig} .2 \mathrm{C}(\mathrm{I})]$ is measured by quantum state tomography. The Bell state is then written into the quantum memories $\mathrm{M}_{1}$ and $\mathrm{M}_{2}$ by an iSWAP pulse (step II) ${ }^{22}$, leaving the qubits in their ground state $|\mathrm{g}\rangle$, with density matrix $\hat{\rho}_{(\mathrm{II})}$ [Fig. 2C (II)]. While storing the first Bell state in $\mathrm{M}_{1}$ and $\mathrm{M}_{2}$, a second Bell state with density matrix 

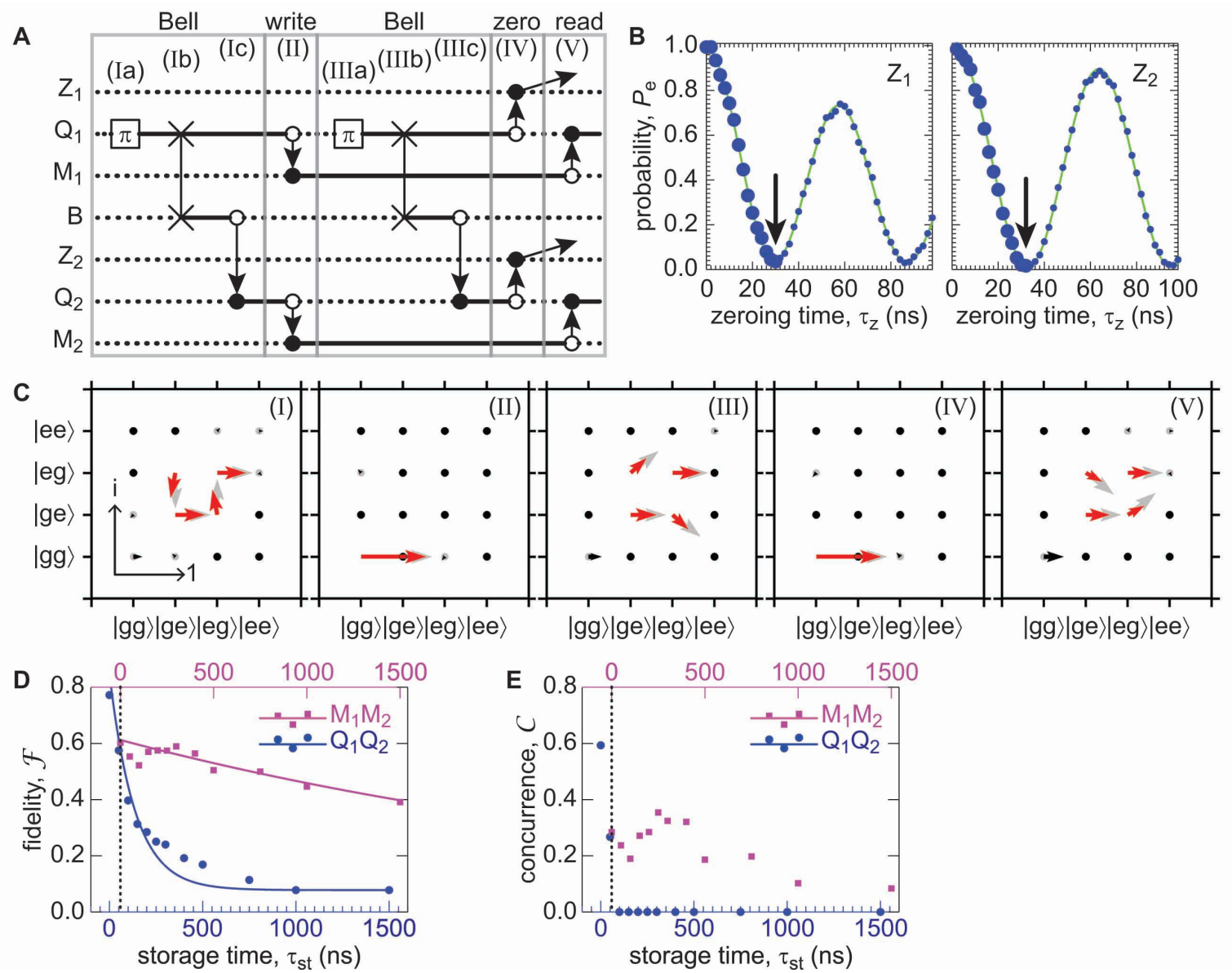

Figure 2: Programming the quantum von Neumann architecture. (A) Quantum algorithm comprising 7 independent channels interacting through five computational steps. Dotted and solid lines represent channels in the ground and excited/superposition states, respectively. A black rectangle represents a $\pi$-pulse; two crosses connected by a solid line a $\sqrt{\text { iSWAP; }}$, an open and a closed circle connected by a single arrow an iSWAP; oblique arrows indicate decay from a zeroing register. (B) Calibration of the zeroing gates. Each qubit is prepared in $|\mathrm{e}\rangle$, interacts on resonance with its zeroing register for a time $\tau_{\mathrm{z}}$, and its probability $P_{\mathrm{e}}$ measured, with $P_{\mathrm{e}}$ plotted vs. $\tau_{\mathrm{z}}$ (large and small blue circles). The solid green line is a decaying cosine fit to the data. The black arrows indicate the zeroing time for each qubit. (C) Density matrices $\hat{\rho}_{(\mathrm{I})}, \hat{\rho}_{(\mathrm{II})}, \ldots, \hat{\rho}_{(\mathrm{V})}$ of the $\mathrm{Q}_{1}-\mathrm{Q}_{2}$ state for each step in A (scale key on bottom left). Grey arrows: Ideal state. Red and black arrows and black dots: Measured state (black arrows indicate errors). The off-diagonal elements of $\hat{\rho}_{(\mathrm{II})}, \hat{\rho}_{(\mathrm{III})}$, and $\hat{\rho}_{(\mathrm{V})}$ have different angles because of dynamic phases 26 . Fidelities: $\mathcal{F}_{(\mathrm{II})}=0.772 \pm 0.003, \mathcal{F}_{\text {(II) }}=0.916 \pm 0.002, \mathcal{F}_{\text {(III) }}=0.689 \pm 0.003, \mathcal{F}_{(\mathrm{IV})}=0.913 \pm 0.002$, and $\mathcal{F}_{(\mathrm{V})}=0.606 \pm 0.003$. Concurrences: $\mathcal{C}_{(\mathrm{I})}=0.593 \pm 0.006, \mathcal{C}_{\text {(II) }}=0.029 \pm 0.005, \mathcal{C}_{\text {(III) }}=0.436 \pm 0.007, \mathcal{C}_{(\mathrm{IV})}=0.019 \pm 0.005$, and $\mathcal{C}_{\mathrm{V}}=0.345 \pm 0.008$. (D) Comparison of fidelity $\mathcal{F}$ as a function of storage time $\tau_{\text {st }}$ for a Bell state stored in $\mathrm{Q}_{1}$ and $\mathrm{Q}_{2}$ (blue circles) vs. that stored in $\mathrm{M}_{1}$ and $\mathrm{M}_{2}$ (magenta squares; error bars smaller than symbols). The solid lines are exponential fits to data. (E) As in D, but for the concurrence $\mathcal{C}$. In D and $\mathrm{E}$ the vertical black dotted line indicates the time delay $(\simeq 59 \mathrm{~ns})$ associated with memory storage, with respect to storage in the qubits, due to the writing and reading operations (II) and (V) in A.

$\hat{\rho}_{\text {(III) }}[$ Fig. 2C (III)] is created between the qubits, using a sequence similar to the first operation (step III, a to c).

In order to re-use the qubits $\mathrm{Q}_{1}$ and $\mathrm{Q}_{2}$, for example to read out the quantum information stored in the memories $\mathrm{M}_{1}$ and $\mathrm{M}_{2}$, the second Bell state has to be dumped ${ }^{23}$. This is accomplished using two zeroing gates, by bringing $\mathrm{Q}_{1}$ on resonance with $\mathrm{Z}_{1}$ and $\mathrm{Q}_{2}$ with $\mathrm{Z}_{2}$ for a zeroing time $\tau_{\mathrm{z}}$, corresponding to a full iSWAP (step IV). Figure $2 \mathrm{~B}$ shows the corresponding dynamics, where each qubit, initially in the excited state $|\mathrm{e}\rangle$, is measured in the ground state $|\mathrm{g}\rangle$ after $\simeq 30 \mathrm{~ns}$. The density matrix $\hat{\rho}_{(\mathrm{IV})}$ of the zeroed two-qubit system is shown in Fig. $2 \mathrm{C}$ (IV). Once zeroed, the qubits can be used to read the memories (step V), allowing us to verify that, at the end of the algorithm, the stored state is still entangled. This is clearly demonstrated by the density matrix shown in Fig. $2 \mathrm{C}(\mathrm{V})$.

The ability to store entanglement in the memories, which are characterized by much longer coherence times than the qubits, is key to the quantum von Neumann architecture. We demonstrate this capability in Fig. 2, D and E, where the fidelity and concurrence metrics ${ }^{6}$ of the Bell states stored in $\mathrm{M}_{1}$ and $\mathrm{M}_{2}$ are compared to those for the same states stored in $\mathrm{Q}_{1}$ and $\mathrm{Q}_{2}$. The experiment is performed as in Fig. 2A, but eliminating steps (III) and 
A

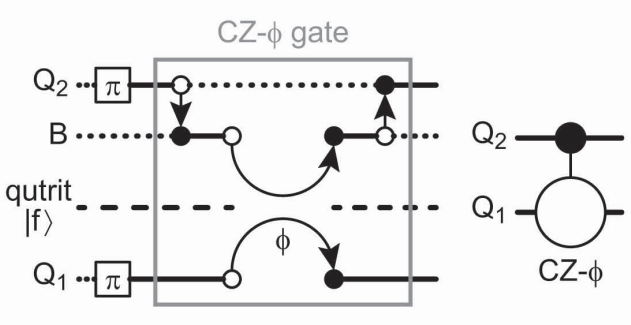

B $\quad 0.0 \quad 0.5 \quad 1.0 P_{\mathrm{e}}$

C

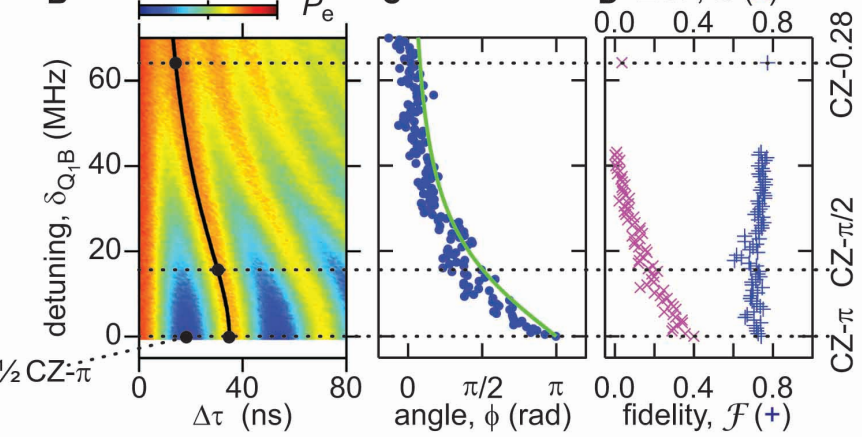

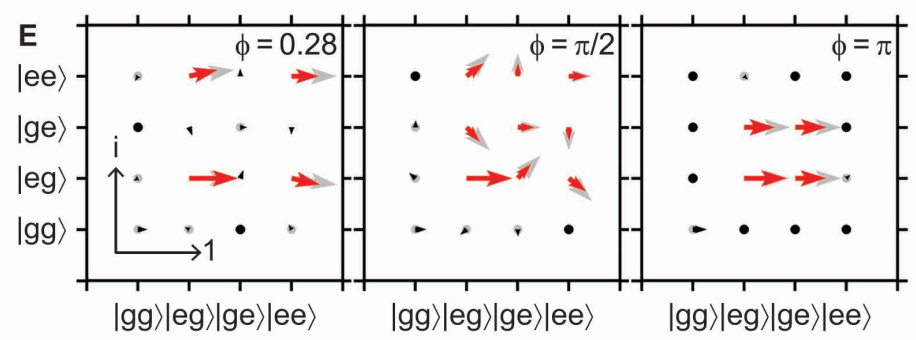

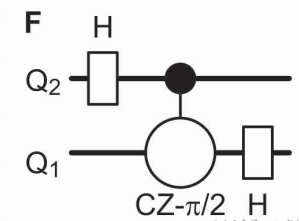

$|g g\rangle|e g\rangle|g e\rangle|e e\rangle$

$|g g\rangle|\operatorname{eg}\rangle|g e\rangle|e e\rangle$

$|g g\rangle|\operatorname{eg}\rangle|g e\rangle|e e\rangle$

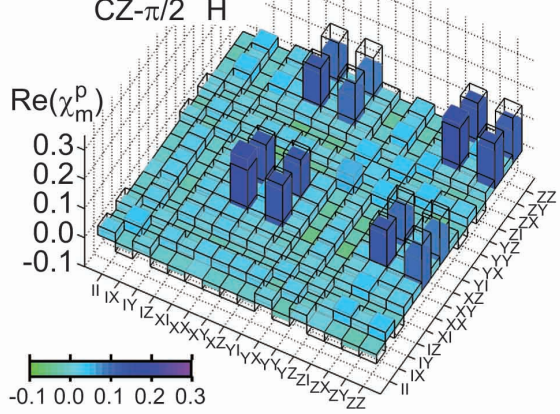

Figure 3: The quantum Fourier transform. (A) (Left) Quantum logic circuit of a CZ- $\phi$ gate (enclosed in a grey box) for $\left|\mathrm{Q}_{1} \mathrm{Q}_{2}\right\rangle=|\mathrm{ee}\rangle$. The $|f\rangle$ state of $Q_{1}$ is indicated by a dashed line. The process where $Q_{1}$ acquires the phase $\phi$ is represented by a pair of open/closed circles, connected by a single arrow in an arc shape. All other symbols are as in Fig. 2A. (Right) Shorthand symbol for the CZ- $\phi$ gate. Although the gate unitary matrix is symmetric, the symbol shows the asymmetric implementation of the gate. (B) Time-domain swaps between the states $\left|\mathrm{Q}_{1} \mathrm{~B}\right\rangle=|\mathrm{e} 1\rangle$ and $|\mathrm{f} 0\rangle$, where we plot the probability $P_{\mathrm{e}}$ (color scale) vs. interaction time $\Delta \tau$ and detuning $\delta_{\mathrm{Q}_{1} \mathrm{~B}}$. The solid black line indicates combinations of interaction time and detuning that completely depopulate the non-computational $|\mathrm{f}\rangle$ state. The three black dots on this line correspond to a CZ- $\pi, \mathrm{CZ}-\pi / 2$, and CZ- 0.28 gate (see far right). The fourth black dot (outside the line) corresponds to a $1 / 2 \mathrm{CZ}-\pi$ gate (see bottom-left), where the $|\mathrm{e}\rangle$ state has been shelved to the non-computational $|\mathrm{f}\rangle$ state. (C) Phase $\phi$ acquired by $\mathrm{Q}_{1}$ as a function of $\delta_{\mathrm{Q}_{1} \mathrm{~B}}$. The blue dots indicate experimental data and the solid green line the theory of Eq. 1126. (D) Fidelity $\mathcal{F}$ (blue " + " symbols) and EOF (magenta " $\times$ " symbols) of measured density matrices $\hat{\rho}_{\phi}$ vs. $\delta_{\mathrm{Q}_{1} \mathrm{~B}}$. (E) (Left to Right) Density matrices $\hat{\rho}_{\phi}=\hat{\rho}_{0.28}, \hat{\rho}_{\pi / 2}$, and $\hat{\rho}_{\pi}$, obtained when $\phi=0.28, \phi=\pi / 2$, and $\phi=\pi \mathrm{rad}$ in Eq. 2 (scale key on bottom left). The arrows are color-coded as in Fig. 2C. The measured fidelities are $\mathcal{F}_{0.28}=0.751 \pm 0.064, \mathcal{F}_{\pi / 2}=0.735 \pm 0.017$, and $\mathcal{F}_{\pi}=0.741 \pm 0.030$, and EOF are $\mathcal{E}_{0.28}=0.020 \pm 0.055$ (lower bound $\mathcal{E}_{0.28}=0$ ), $\mathcal{E}_{\pi / 2}=0.106 \pm 0.031$, and $\mathcal{E}_{\pi}=0.401 \pm 0.062$. (F) (Top-Left) Logic circuit for a two-qubit quantum Fourier transform and, (Bottom), real part of the corresponding $\chi_{\mathrm{m}}^{\mathrm{p}}$ matrix 25 . The process fidelity for the real and imaginary (not shown) part of $\chi_{\mathrm{m}}^{\mathrm{p}}$ is $\mathcal{F}_{\chi}=0.657 \pm 0.014$. The confidence intervals are estimated from 10 measurements for $\hat{\rho}_{0.28}, 6$ for $\hat{\rho}_{\pi / 2}$ and $\hat{\rho}_{\pi}$, and 15 for $\chi_{\mathrm{m}}^{\mathrm{p}}$.

(IV). For the qubits, the storage time $\tau_{\text {st }}$ is defined as the wait time at the end of step (I), prior to measuring the qubit states, whereas for the resonators the wait time is that between the write and read steps. The fidelity of the qubit states decays to below 0.2 after $400 \mathrm{~ns}$, while for the states stored in the memories it remains above 0.4 up to $\simeq 1.5 \mu \mathrm{s}$. Most importantly, after only $100 \mathrm{~ns}$ the state stored in the qubits does not preserve any entanglement, as indicated by a zero concurrence, whereas the memories retain their entanglement for at least $1.5 \mu \mathrm{s}$ (Fig. 2E). We expect taking advantage of our architecture in long computations, where qubit states can be protected and reused by writing them into, and reading them out of, the long-lived quRAM.

Two-qubit universal gates are a vital resource for the operation of the quCPU23. A variety of such gates have been implemented in superconducting circuits $5 / 15]-17 \cdot 19[20$, with some recent demonstrations of quantum al-

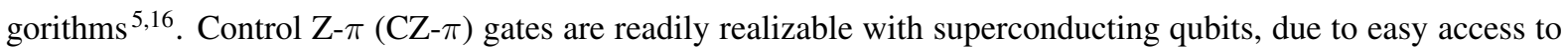
the third energy state of the qubit, effectively operating the qubit as a qutrit ${ }^{5|16| 20 \mid 25}$. However, CZ- $\pi$ gates are just a subset of the more general class of CZ- $\phi$ gates, obtained for the special case where the phase $\phi=\pi$. In our architecture, the full class of CZ- $\phi$ gates, with $\phi$ from $\simeq 0$ to $\pi$, can be generated by coupling a qutrit close to 
resonance with a bus resonator.

Figure 3A shows the quantum logic circuit that generates the CZ- $\phi$ gate (Left) and a shorthand symbol for the gate (Right). The logic circuit demonstrates the nontrivial case where qubits $\mathrm{Q}_{1}$ and $\mathrm{Q}_{2}$ are brought from their initial ground state to $\left|\mathrm{Q}_{1} \mathrm{Q}_{2}\right\rangle=\mid$ ee $\rangle$ by applying a $\pi$-pulse to each qubit. The excitation in $\mathrm{Q}_{2}$ is then transferred into bus resonator $\mathrm{B}$, and $\mathrm{Q}_{1}$ 's $|\mathrm{e}\rangle \leftrightarrow|\mathrm{f}\rangle$ transition brought close to resonance with $\mathrm{B}$ for the time required for a $2 \pi$-rotation, where the states $\left|\mathrm{Q}_{1} \mathrm{~B}\right\rangle=|\mathrm{e} 1\rangle$ and $|\mathrm{f} 0\rangle$ are detuned by a frequency $\delta_{\mathrm{Q}_{1} \mathrm{~B}}$, which we term a "semi-resonant condition." In this process $\mathrm{Q}_{1}$ acquires the phase ${ }^{26}$

$$
\phi=\pi-\pi \frac{\delta_{\mathrm{Q}_{1} \mathrm{~B}}}{\sqrt{\delta_{\mathrm{Q}_{1} \mathrm{~B}}^{2}+\tilde{g}_{\mathrm{Q}_{1} \mathrm{~B}}^{2}}},
$$

where $\tilde{g}_{\mathrm{Q}_{1} \mathrm{~B}}$ is the coupling frequency between $|\mathrm{e} 1\rangle$ and $|\mathrm{f} 0\rangle$. The final step is to move the excitation from B back into $\mathrm{Q}_{2}$.

The time-domain swaps of $\left|\mathrm{Q}_{1} \mathrm{~B}\right\rangle$ between the states $|\mathrm{e} 1\rangle$ and $|\mathrm{f} 0\rangle$ are shown in Fig. 3B, where the solid black line indicates the detunings and corresponding interaction times used to generate any phase $0 \lesssim \phi \leqslant \pi$ (ideally $\phi \rightarrow 0$ when $\delta_{\mathrm{Q}_{1} \mathrm{~B}} \rightarrow \infty$ ). These phases are measured by performing two Ramsey experiments on $\mathrm{Q}_{1}$ for each value of the detuning $\delta_{\mathrm{Q}_{1} \mathrm{~B}}$, one with $\mathrm{B}$ in the $|0\rangle$ state, and the other with $\mathrm{B}$ in the $|1\rangle$ state. The relative phase between the Ramsey fringes corresponds to the value of $\phi$ for the CZ- $\phi$ gate ${ }^{26}$, as shown in Fig. 3C.

A more sophisticated version of this experiment is performed by initializing $\mathrm{Q}_{1}$ and $\mathrm{Q}_{2}$ each in the superposition state $|\mathrm{g}\rangle+|\mathrm{e}\rangle$. We move $\mathrm{Q}_{2}$ 's state into $\mathrm{B}$, perform a CZ- $\phi$ gate with $0 \lesssim \phi \leqslant \pi$, move the state in B back into $\mathrm{Q}_{2}$, rotate $\mathrm{Q}_{1}$ 's resulting state by $\pi / 2$ about the $y$-axis, and perform a joint measurement of $\mathrm{Q}_{1}$ and $\mathrm{Q}_{2}$. Ideally, this protocol permits to create two-qubit states ranging from a product state for $\phi=0$ to a maximally-entangled state for $\phi=\pi$. In the two-qubit basis set $\mathcal{M}_{2}=\{|\mathrm{gg}\rangle,|\mathrm{eg}\rangle,|\mathrm{ge}\rangle,|\mathrm{ee}\rangle\}$, the general density matrix of such two-qubit states reads

$$
\hat{\rho}_{\phi}=\left(\begin{array}{cccc}
0 & 0 & 0 & 0 \\
0 & 1 / 2 & \left(1-e^{-i \phi}\right) / 4 & \left(1+e^{-i \phi}\right) / 4 \\
0 & \left(1-e^{i \phi}\right) / 4 & (1-\cos \phi) / 4 & (-i \sin \phi) / 4 \\
0 & \left(1+e^{i \phi}\right) / 4 & (i \sin \phi) / 4 & (1+\cos \phi) / 4
\end{array}\right)
$$

Figure 3D shows the fidelity and entanglement of formation $(\mathrm{EOF})^{\sqrt{6}}$ of two-qubit states generated using 70 values of $\phi$. Figure 3E shows three examples of $\hat{\rho}_{\phi}$ for $\phi=0.28, \phi=\pi / 2$, and $\phi=\pi$, respectively.

The state generated using $\phi=\pi / 2$ plays a central role in the implementation of the two-qubit quantum Fourier transform. Neglecting bit-order reversal, the quantum Fourier transform can be realized by applying a Hadamard gate to $\mathrm{Q}_{2}$, followed by a CZ- $\pi / 2$ gate between $\mathrm{Q}_{1}$ and $\mathrm{Q}_{2}$, and finally a Hadamard on $\mathrm{Q}_{1}$ [2719, as sketched in Fig. 3F (Top-Left). Representing the input state of the transform as $|x\rangle$ (position) and the output as $|p\rangle$ (momentum), assuming $|x\rangle \in \mathcal{M}_{2}$ and the indexes $x$ and $p$ are integers, with $p \in\{0,1,2,3\}$, the output state $|p\rangle=\sum_{x=0}^{3} e^{i 2 \pi x p / 4}|x\rangle / 2$, corresponding to a $4 \times 4$ unitary operator. This operator can be fully characterized by means of quantum process tomography ${ }^{255}$, which allows us to obtain the $\chi_{\mathrm{m}}^{\mathrm{p}}$ matrix ${ }^{215}$ shown in Fig. $3 \mathrm{~F}$ (Bottom).

Finally, by combining the CZ- $\phi$ and zeroing gates, we can implement a Toffoli-class gate ${ }^{10 \mid 1227}$, the threequbit OR phase gate. This gate, combined with single qubit rotations, is sufficient for universal computation. A Toffoli gate is a doubly-controlled quantum operation, where a unitary operation is applied to a target qubit subject to the state of two control qubits. The canonical Toffoli is a doubly-controlled NOT gate; here we consider a doubly-controlled phase gate, which is equivalent through a change of basis of the target qubit. In the canonical Toffoli gate, the control gate is applied if both control qubits, $\mathrm{Q}_{1}$ AND $\mathrm{Q}_{2}$, are in state $|e\rangle$. In our case, the control gate is applied conditionally if the controls $\mathrm{Q}_{1} \mathrm{OR} \mathrm{Q}_{2}$ are in $|\mathrm{e}\rangle$. Additionally, we have implemented a three-qubit gate for the logical function XOR, which, even though not a Toffoli-class gate, helps to understand the 

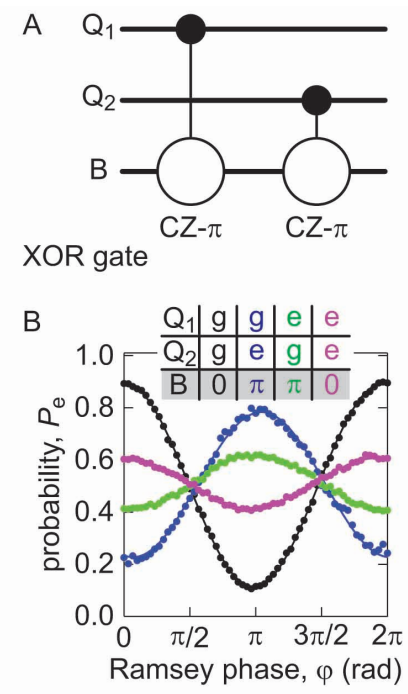

C $\phi_{|I m n\rangle}-\phi_{|g g 0\rangle}(\mathrm{rad})$

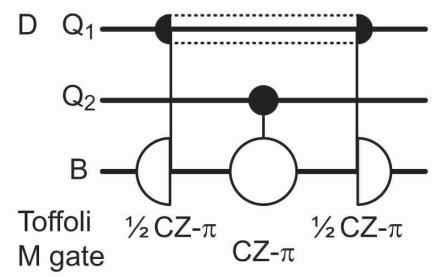

E
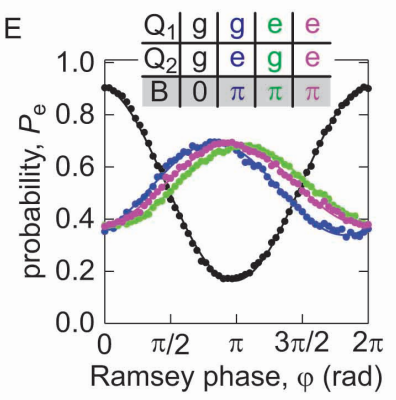

$\mathrm{F} \quad \phi_{|I m n\rangle}-\phi_{|\mathrm{gg} 0\rangle}(\mathrm{rad})$ $-\pi / 2 \quad 0 \quad \pi / 2 \quad \pi \quad 3 \pi / 2$

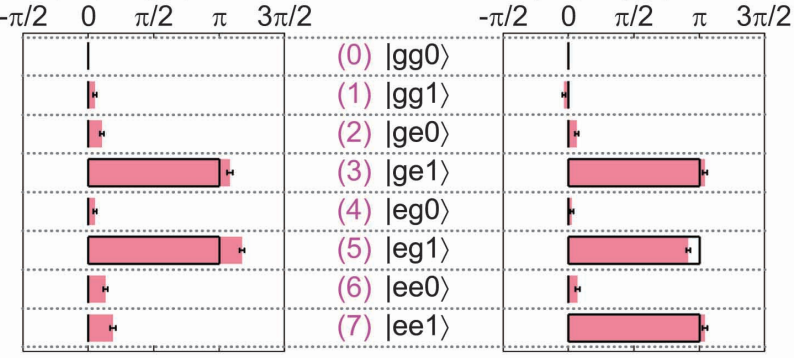

Figure 4: Three-qubit gates: The XOR phase gate and the Toffoli-class M gate. (A) Quantum logic circuit for the XOR phase gate. (B) (Top) XOR-gate truth table. (Bottom) Ramsey fringes associated with the truth table, showing the probability $P_{\mathrm{e}}$ of measuring $\mathrm{Q}_{2}$ in $|\mathrm{e}\rangle$, vs. the Ramsey phase $\varphi$, for the control input states in $\mathcal{M}_{2}$. Black and magenta dots: 0 phase. Blue and green dots: $\pi$ phase. The solid lines are least-squares fits to the data used to extract the truth-table phases. (C) Quantum phase tomography for the XOR gate: Phase $\phi_{|l m n\rangle}-\phi_{|g g 0\rangle}$, for each state $|\operatorname{lmn}\rangle \in \mathcal{M}_{3}$. Black open boxes: Ideal values. Pink areas: Measured values with corresponding confidence intervals (black lines). (D) Quantum logic circuit for the $\mathrm{M}$ gate, implemented as a $1 / 2 \mathrm{CZ}-\pi$ gate (cf. Fig. 3B) between $\mathrm{Q}_{1}$ and B (half-dot/half-open circle connected by solid line), followed by a CZ- $\pi$ gate between $Q_{2}$ and $B$, and a second $1 / 2 C Z-\pi$ gate between $Q_{1}$ and $B$. The dotted black lines connecting the two $1 / 2 \mathrm{CZ}-\pi$ gates indicate qubit shelving to the $|\mathrm{f}\rangle$ state. (E) As in panel $\mathrm{B}$, but for the $\mathrm{M}$ gate. (F) As in $\mathrm{C}$, but for the $\mathrm{M}$ gate.

more complex OR gate.

The quantum logic circuits for the XOR and OR gates are drawn in Fig. 4, A and D. The control qubits are $\mathrm{Q}_{1}$ and $\mathrm{Q}_{2}$ and the target is the bus resonator $\mathrm{B}$, effectively acting as the third qubit (as only the states $|0\rangle$ and $|1\rangle$ of $\mathrm{B}$ are used). The XOR gate is realized as a series of two CZ- $\pi$ gates between the controls and the target, and the OR gate as the series $1 / 2 \mathrm{CZ}-\pi, \mathrm{CZ}-\pi$, and $1 / 2 \mathrm{CZ}$ - $\pi$, in an "M-shape" configuration.

The truth table for the XOR gate is displayed in Fig. 4B (Top). The control qubits $\mathrm{Q}_{1}$ and $\mathrm{Q}_{2}$ are assumed to be in one of the states in $\mathcal{M}_{2}$, while the target $\mathrm{B}$ is in $|0\rangle+|1\rangle$. The target acquires a phase $\pi$, corresponding to a "true" result, only when the controls are in the state $\left|\mathrm{Q}_{1} \mathrm{Q}_{2}\right\rangle=|\mathrm{ge}\rangle$ or $|\mathrm{eg}\rangle$. For the other non-trivial case $\left|\mathrm{Q}_{1} \mathrm{Q}_{2}\right\rangle=|\mathrm{ee}\rangle$, the target acquires 0 phase, corresponding to a "false" result. This is due to the action of the two CZ- $\pi$ gates, giving a global phase $\pi$ when either of the controls is in $|\mathrm{e}\rangle$, and a phase $2 \pi$ (equivalent to a 0 phase) when both are in $|\mathrm{e}\rangle$.

The truth table can be experimentally measured by performing Ramsey experiments on the target, one for each pair of control states. The experiments are realized by, $(i)$, preparing $\mathrm{Q}_{2}$ in the superposition state $|\mathrm{g}\rangle+|\mathrm{e}\rangle$ by means of a $\pi / 2$-pulse; (ii), moving the state from $\mathrm{Q}_{2}$ into $\mathrm{B}$, thus creating a $|0\rangle+|1\rangle$ state in $\mathrm{B}$; (iii), preparing $\mathrm{Q}_{1}$ and $\mathrm{Q}_{2}$ in each possible pair of control states in $\mathcal{M}_{2}$ by means of $\pi$-pulses; (iv), performing the XOR gate; $(v)$, zeroing $\mathrm{Q}_{2}$ into $\mathrm{Z}_{2}$ at the end of the XOR gate; (vi), moving the final target state from $\mathrm{B}$ into the zeroed $\mathrm{Q}_{2}$; (vii), completing the Ramsey sequence on $\mathrm{Q}_{2}$ with a second $\pi / 2$-pulse with variable rotation axis relative to the pulse 
in (i). The measurement outcomes are displayed in Fig. 4B (Bottom), together with the least-squares fits used to extract the phase information associated with each value of the truth table. The Ramsey fringes for the two control states $|\mathrm{ge}\rangle$ and $|\mathrm{eg}\rangle$ are inverted relative to the reference state $|\mathrm{gg}\rangle$, as expected from the XOR gate truth table.

In general, given the $\mathrm{Q}_{1}-\mathrm{Q}_{2}-\mathrm{B}$ basis set $\mathcal{M}_{3}=\{|\operatorname{gg} 0\rangle,|\operatorname{gg} 1\rangle,|\operatorname{ge} 0\rangle,|\operatorname{ge} 1\rangle,|\operatorname{eg} 0\rangle$, $\mid$ eg 1$\rangle, \mid$ ee 0$\rangle, \mid$ ee 1$\rangle\}$, the vector $\tau^{\mathrm{XOR}}$ of the diagonal elements associated with the ideal unitary matrix of the XOR gate reads

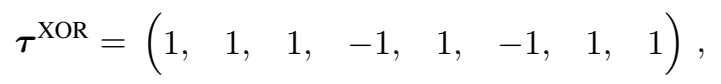

while all off-diagonal elements of the matrix are zero. Each element $\tau_{k}^{\mathrm{XOR}}$ can be expressed as a complex expo-

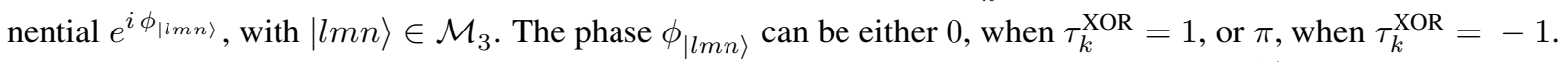
Among the eight values of $\phi_{|l m n\rangle}$, only seven are physically independent, as the element $e^{\left.i \phi_{\mid g g 0}\right\rangle}$ can be factored, reducing the set of possible phases to $\phi_{|l m n\rangle}-\phi_{|\operatorname{gg} 0\rangle}$, with $|l m n\rangle \in \mathcal{M}_{3}-\{|\operatorname{gg} 0\rangle\}$.

In analogy to the truth-table for the target $\mathrm{B}$, a table with four phase differences can also be obtained for the controls $\mathrm{Q}_{1}$ and $\mathrm{Q}_{2}$, resulting in a total of twelve phase differences. These differences can be measured by performing Ramsey experiments both on the target and the control qubits. It can be shown that from the twelve phase differences, one can obtain the seven independent phases associated with the diagonal elements $\tau_{k}^{\mathrm{XOR} 26}$, thus realizing a quantum phase tomography of the Toffoli gate ${ }^{28}$. Figure $4 \mathrm{C}$ displays the phase tomography results for our experimental implementation of the XOR gate.

The truth table associated with the M gate is reported in Fig. 4E (Top), where the only difference from the XOR gate is the phase $\pi$ acquired by the target $\mathrm{B}$ when the controls $\mathrm{Q}_{1}$ and $\mathrm{Q}_{2}$ are loaded in state $\left|\mathrm{Q}_{1} \mathrm{Q}_{2}\right\rangle=|\mathrm{ee}\rangle$. In this case, the action of the first ${ }^{1} / 2 \mathrm{CZ}-\pi$ gate between $\mathrm{Q}_{1}$ and $\mathrm{B}$ shelves the $|1\rangle$ state from $\mathrm{B}$ to the non-computational state $|\mathrm{f}\rangle$ in $\mathrm{Q}_{1}$, where it remains until the second ${ }^{1} / 2 \mathrm{CZ}-\pi$ gate. Moving the state of $\mathrm{Q}_{1}$ outside the computational space during the intermediate $\mathrm{CZ}-\pi$ gate between $\mathrm{Q}_{2}$ and $\mathrm{B}$ effectively turns off the $\mathrm{CZ}-\pi$ gate ${ }^{1229}$. The target B thus only acquires a total phase $\pi$ due to the combined action of the two $1 / 2 \mathrm{CZ}-\pi$ gates (cf. Fig. 4D). The experimental truth table obtained from Ramsey fringes is shown in Fig. 4E (Bottom).

The vector $\tau^{\mathrm{M}}$ of the diagonal elements associated with the ideal unitary matrix of the $\mathrm{M}$ gate is $\tau^{\mathrm{M}}=$ $(1,1,1,-1,1,-1,1,-1)$. A similar procedure as for the XOR gate allows us to obtain the quantum phase tomography of the M gate (Fig. 4F).

Quantum phase tomography makes it possible to define the phase fidelity of the XOR and M gate,

$$
\mathcal{F}_{\varphi} \equiv 1-\frac{\varepsilon_{\varphi}}{\pi}
$$

where $\varepsilon_{\varphi}$ is the gate root-mean-square phase error, with an upper bound of $\pi$. For the XOR gate we find that $\mathcal{F}_{\varphi}=0.954 \pm 0.004$, and for the $\mathrm{M}$ gate $\mathcal{F}_{\varphi}=0.979 \pm 0.003$.

Our results provide optimism for the near-term implementation of a larger-scale quantum processor ${ }^{1 / \sqrt{3}}$ based on superconducing circuits. Our architecture shows that proof-of-concept factorization algorithms ${ }^{[2 / 35]}$ and simple quantum error correction codes ${ }^{\sqrt{231618}}$ might be achievable using this approach.

\section{References}

1. D. P. DiVincenzo, The physical implementation of quantum computation. Fortschr. Phys. 48, 771-783 (2000).

2. M. A. Nielsen \& I. L. Chuang, Quantum computation and quantum information. (Cambridge Univ. Press, Cambridge - UK, 2000).

3. N. D. Mermin, Quantum computer science: An introduction. (Cambridge Univ. Press, Cambridge - UK, 2007).

4. H. M. Wiseman \& G. J. Milburn, Quantum measurement and control. (Cambridge Univ. Press, Cambridge UK, 2010). 
5. L. M. K. Vandersypen, M. Steffen, G. Breyta, C. S. Yannoni, M. H. Sherwood \& I. L. Chuang, Experimental realization of Shor's quantum factoring algorithm using nuclear magnetic resonance. Nature (London) 414, 883-887 (2001).

6. D. G. Cory, M. D. Price, W. Maas, E. Knill, R. Laflamme, W. H. Zurek, T. F. Havel \& S. S. Somaroo, Experimental quantum error correction. Phys. Rev. Lett. 81, 2152-2155 (1998).

7. Y. S. Weinstein, M. A. Pravia, E. M. Fortunato, S. Lloyd \& D. G. Cory, Implementation of the quantum Fourier transform. Phys. Rev. Lett. 86, 1889-1891 (2001).

8. R. Blatt \& D. Wineland, Entangled states of trapped atomic ions. Nature (London) 453, 1008-1015 (2008).

9. J. Chiaverini, J. Britton, D. Leibfried, E. Knill, M. D. Barrett, R. B. Blakestad, W. M. Itano, J. D. Jost, C. Langer, R. Ozeri, T. Schaetz \& D. J. Wineland, Implementation of the semiclassical quantum Fourier transform in a scalable system. Science 308, 997-1000 (2005).

10. T. Monz, K. Kim, W. Hänsel, M. Riebe, A. S. Villar, P. Schindler, M. Chwalla, M. Hennrich \& R. Blatt, Realization of the quantum Toffoli gate with trapped ions. Phys. Rev. Lett. 102, 040501 (2009).

11. D. J. Reilly, J. M. Taylor, J. R. Petta, C. M. Marcus, M. P. Hanson \& A. C. Gossard, Suppressing spin qubit dephasing by nuclear state preparation. Science 321, 817-821 (2008).

12. B. P. Lanyon, M. Barbieri, M. P. Almeida, T. Jennewein, T. C. Ralph, K. J. Resch, G. J. Pryde, J. L. O’Brien, A. Gilchrist \& A. G. White, Simplifying quantum logic using higher-dimensional Hilbert spaces. Nature Phys. 5, 134-140 (2009).

13. K. S. Choi, H. Deng, J. Laurat \& H. J. Kimble, Mapping photonic entanglement into and out of a quantum memory. Nature (London) 452, 67-71 (2008).

14. J. Clarke \& F. K. Wilhelm, Superconducting quantum bits. Nature (London) 453, 1031-1042 (2008).

15. J. H. Plantenberg, P. C. de Groot, C. J. P. M. Harmans \& J. E. Mooij, Demonstration of controlled-NOT quantum gates on a pair of superconducting quantum bits. Nature (London) 447, 836-839 (2007).

16. L. DiCarlo, J. M. Chow, J. M. Gambetta, L. S. Bishop, B. R. Johnson, D. I. Schuster, J. Majer, A. Blais, L. Frunzio, S. M. Girvin \& R. J. Schoelkopf, Demonstration of two-qubit algorithms with a superconducting quantum processor. Nature (London) 460, 240-244 (2009).

17. P. J. Leek, S. Filipp, P. Maurer, M. Baur, R. Bianchetti, J. M. Fink, M. Göppl, L. Steffen \& A. Wallraff, Using sideband transitions for two-qubit operations in superconducting circuits. Phys. Rev. B 79, 180511(R) (2009).

18. T. Yamamoto, M. Neeley, E. Lucero, R. C. Bialczak, J. Kelly, M. Lenander, M. Mariantoni, A. D. O’Connell, D. Sank, H. Wang, M. Weides, J. Wenner, Y. Yin, A. N. Cleland \& J. M. Martinis, Quantum process tomography of two-qubit controlled-Z and controlled-NOT gates using superconducting phase qubits. Phys. Rev. B 82, 184515 (2010).

19. M. Neeley, R. C. Bialczak, M. Lenander, E. Lucero, M. Mariantoni, A. D. O’Connell, D. Sank, H. Wang, M. Waides, J. Wenner, Y. Yin, T. Yamamoto, A. N. Cleland \& J. M. Martinis, Generation of three-qubit entangled states using superconducting phase qubits. Nature (London) 467, 570-573 (2010).

20. L. DiCarlo, M. D. Reed, L. Sun, B. R. Johnson, J. M. Chow, J. M. Gambetta, L. Frunzio, S. M. Girvin, M. H. Devoret \& R. J. Schoelkopf, Preparation and measurement of three-qubit entanglement in a superconducting circuit. Nature (London) 467, 574-578 (2010). 
21. M. Mariantoni, H. Wang, R. C. Bialczak, M. Lenander, E. Lucero, M. Neeley, A. D. O’Connell, D. Sank, M. Weides, J. Wenner, T. Yamamoto, Y. Yin, J. Zhao, J. M. Martinis \& A. N. Cleland, Photon shell game in three-resonator circuit quantum electrodynamics. Nature Phys. 7, 287-293 (2011).

22. H. Wang, M. Mariantoni, R. C. Bialczak, M. Lenander, E. Lucero, M. Neeley, A. D. O’Connell, D. Sank, M. Weides, J. Wenner, T. Yamamoto, Y. Yin, J. Zhao, J. M. Martinis \& A. N. Cleland, Deterministic entanglement of photons in two superconducting microwave resonators. Phys. Rev. Lett. 106, 060401 (2011).

23. M. D. Reed, B. R. Johnson, A. A. Houck, L. DiCarlo, J. M. Chow, D. I. Schuster, L. Frunzio \& R. J. Schoelkopf, Fast reset and suppressing spontaneous emission of a superconducting qubit. Appl. Phys. Lett. 96, 203110 (2010).

24. R. Horodecki, P. Horodecki, M. Horodecki \& K. Horodecki, Quantum entanglement. Rev. Mod. Phys. 81, 865-942 (2009).

25. F. W. Strauch, P. R. Johnson, A. J. Dragt, C. J. Lobb, J. R. Anderson \& F. C. Wellstood, Quantum logic gates for coupled superconducting phase qubits. Phys. Rev. Lett. 91, 167005 (2003).

26. Methods are available as supporting material on Science Online.

27. A. Barenco, C. H. Bennett, R. Cleve, D. P. DiVincenzo, N. Margolus, P. Shor, T. Sleator, J. A. Smolin \& H. Weinfurter, Elementary gates for quantum computation. Phys. Rev. A 52, 3457-3467 (1995).

28. A full gate characterization via quantum process tomography was not possible as we could only simultaneously measure two qubits, with the resonator acting as the third qubit.

29. T. C. Ralph, K. J. Resch \& A. Gilchrist, Efficient Toffoli gates using qudits. Phys. Rev. A 75, 022313 (2007).

\section{Acknowledgements}

This work was supported by IARPA under ARO award W911NF-08-1-0336 and under ARO award W911NF-091-0375. M. M. acknowledges support from an Elings Postdoctoral Fellowship. Devices were made at the UC Santa Barbara Nanofabrication Facility, a part of the NSF-funded National Nanotechnology Infrastructure Network. The authors thank A. G. Fowler for useful comments on scalability, and M. H. Devoret and R. J. Schoelkopf for discussions on Toffoli gates.

\section{Author Contributions}

M.M. performed the experiments and analyzed the data. M.M. and H.W. fabricated the sample. T.Y., H.W., and Y.Y. helped with the Fourier transform and M.N. with three-qubit gates. M.M., A.N.C., and J.M.M. conceived the experiment and co-wrote the manuscript. 


\title{
Supplementary Material for
}

\section{Implementing the Quantum von Neumann Architecture with Superconducting Circuits}

\author{
Matteo Mariantoni, ${ }^{1,4, \S}$ H. Wang, ${ }^{1 *}$ T. Yamamoto,,${ }^{1,2}$ M. Neeley, ${ }^{1 \dagger}$ \\ Radoslaw C. Bialczak, ${ }^{1}$ Y. Chen, ${ }^{1}$ M. Lenander,${ }^{1}$ Erik Lucero, ${ }^{1}$ A. D. O’Connell, ${ }^{1}$ \\ D. Sank,${ }^{1}$ M. Weides,${ }^{1 \dagger}$ J. Wenner, ${ }^{1}$ Y. Yin, ${ }^{1}$ J. Zhao, ${ }^{1}$ \\ A. N. Korotkov, ${ }^{3}$ A. N. Cleland,,$^{1,4}$ John M. Martinis ${ }^{1,4, \S}$ \\ ${ }^{1}$ Department of Physics, University of California, Santa Barbara, CA 93106-9530, USA \\ ${ }^{2}$ Green Innovation Research Laboratories, NEC Corporation, Tsukuba, Ibaraki 305-8501, Japan \\ ${ }^{3}$ Department of Electrical Engineering, University of California, Riverside, CA 92521, USA \\ ${ }^{4}$ California NanoSystems Institute, University of California, \\ Santa Barbara, California 93106-9530, USA
}

§To whom correspondence should be addressed. E-mail: matmar@physics.ucsb.edu (M. M.); martinis@physics.ucsb.edu (J. M. M.)

This PDF file includes:

Materials and Methods

Figs. S1 to S12

Tables $\mathrm{S} 1$ to $\mathrm{S} 3$

References

* Present address: Department of Physics, Zhejiang University, Hangzhou 310027, China.

†Present address: Lincoln Laboratory, Massachusetts Institute of Technology, 244 Wood Street, Lexington, MA 02420-9108, USA.

$\ddagger$ Present address: National Institute of Standards and Technology, Boulder, CO 80305, USA. 


\section{Contents}

Materials and Methods 12

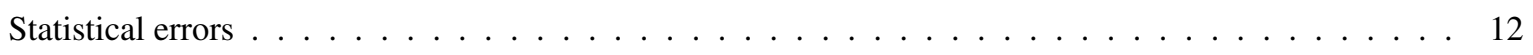

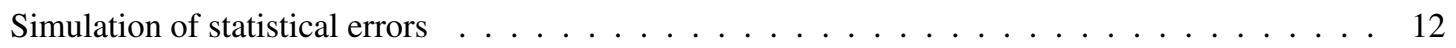

Experimental estimation of statistical errors $\ldots \ldots \ldots \ldots \ldots \ldots$

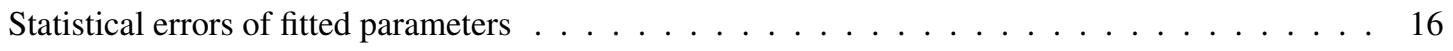

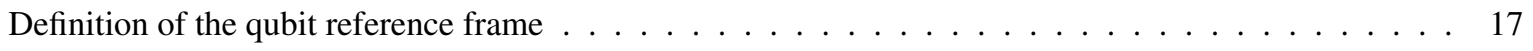

Programming the quantum von Neumann architecture $\ldots \ldots \ldots \ldots \ldots \ldots \ldots$

The quantum Fourier transform $\ldots \ldots \ldots \ldots \ldots \ldots \ldots$

Analytical expression of the phase $\phi$ of a CZ- $\phi$ gate $\ldots \ldots \ldots \ldots \ldots \ldots \ldots$

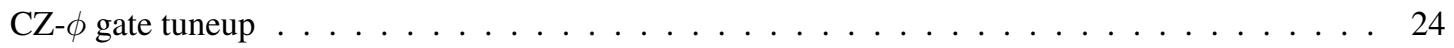

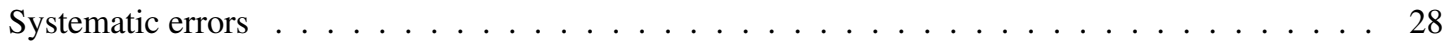

XOR gate and $\mathrm{M}$ gate tuneup $\ldots \ldots \ldots \ldots \ldots \ldots \ldots \ldots$

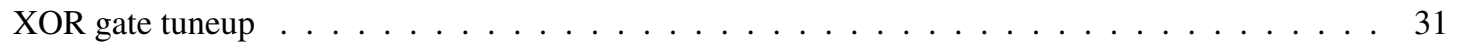

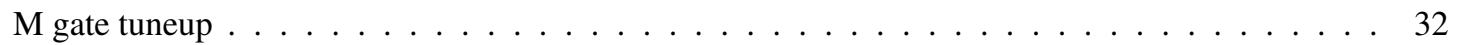

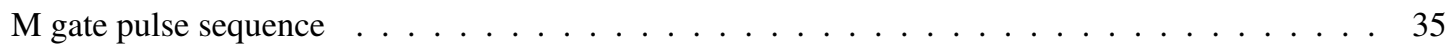

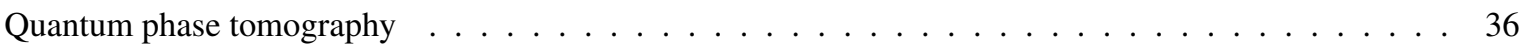



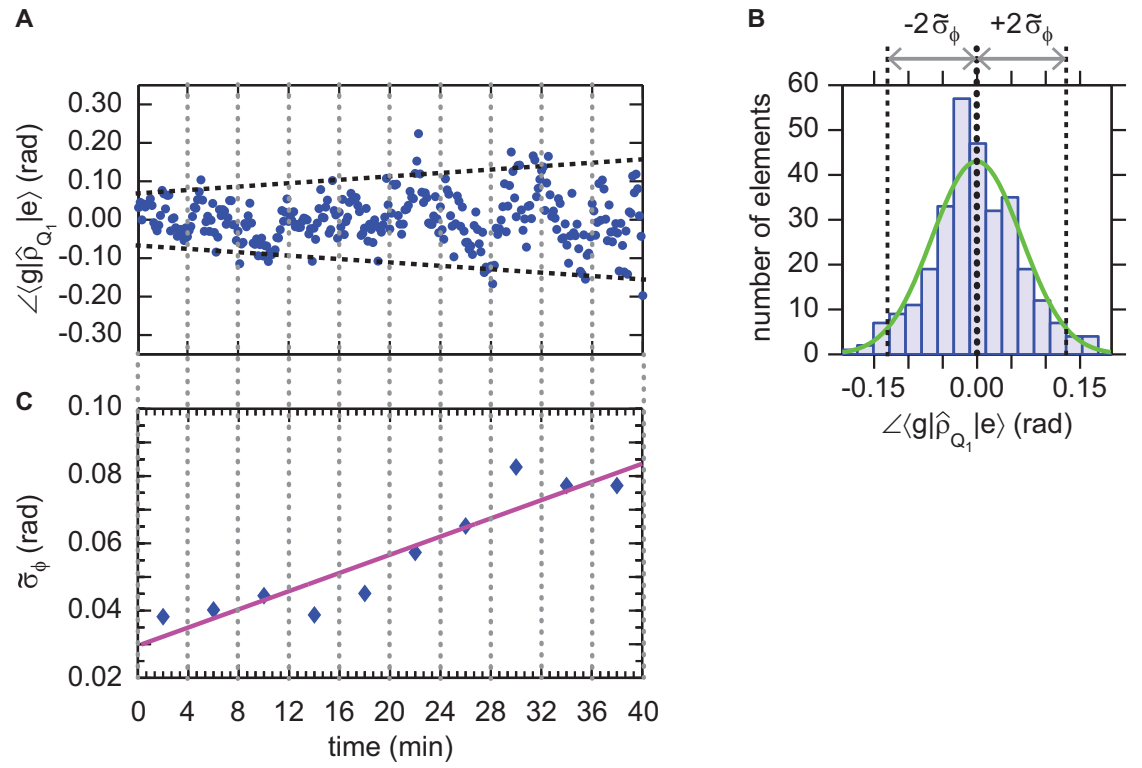

Figure S1: Analysis of phase errors. (A) Phase angle $\angle\left\langle\mathrm{g}\left|\hat{\rho}_{\mathrm{Q}_{1}}\right| \mathrm{e}\right\rangle$ associated with the off-diagonal elements of the matrix $\hat{\rho}_{\mathrm{Q}_{1}}$ of Eq. S7 plotted vs. time. The time axis indicates when the QST of each density matrix $\hat{\rho}_{\mathrm{Q}_{1}}$ was completed. The dashed black lines are a guide-to-theeye showing an increase with time in the data scatter. (B) Histogram associated with the time-trace data in A, plotting the number of elements in the time-trace vs. the phase angle $\angle\left\langle\mathrm{g}\left|\hat{\rho}_{\mathrm{Q}_{1}}\right| \mathrm{e}\right\rangle$. The solid green line is a fit to a normal distribution with mean value of 0 rad and standard deviation $\tilde{\sigma}_{\phi} \simeq 0.065 \mathrm{rad}$. The $\pm 2 \tilde{\sigma}_{\phi}$ window is indicated. (C) Time-bin average of the data in A, showing the value of $\tilde{\sigma}_{\phi}$ for each time-bin of $4 \mathrm{~min}$, for a total of 10 bins (blue diamonds). The bins are indicated by vertical dotted grey lines, which extend to A for clarity. The solid magenta line is a linear fit to the data. This fit was used to estimate the phase errors associated with QST measurements (tomo and octomo).

\section{Materials and Methods}

\section{Statistical errors}

In this section, we analyze the statistical properties of the experimental data shown in the main text. First, we explain how to simulate statistical errors. This procedure was used to estimate the confidence intervals for the data of Fig. 2 in the main text. Second, we describe how statistical errors were obtained from statistical ensembles of independent measurements. This procedure was used for the data of Fig. 3 in the main text. Third, we discuss the estimation of statistical errors due to fits to the data. This procedure was used for the data of Fig. 4 in the main text.

\section{Simulation of statistical errors}

In this subsection, we discuss two important sources of statistical errors in our data: Errors associated with qubit's measurement (binomial-type errors) and errors due to jitter/fluctuations in the electronics (phase errors). Assuming binomial-type and phase errors, we describe the procedures used to simulate the confidence intervals for the elements and metrics of the density matrices shown in Fig. $2 \mathrm{C}$ of the main text.

(i) Binomial-type errors are inherent to our qubit measurement process, where the measurement is repeated a fixed number of times $N$, each measurement trial has two possible outcomes, i.e., qubit being in the ground state $|\mathrm{g}\rangle$ with probability $p_{\mathrm{g}}$ or in the excited state $|\mathrm{e}\rangle$ with probability $p_{\mathrm{e}}=1-p_{\mathrm{g}}$, the probability $p_{\mathrm{e}}$ is to good approximation the same for each trial, and the trials can be considered to be statistically independent. The measurement outcome associated with $|\mathrm{g}\rangle$ is counted as 0 , and that associated with $|\mathrm{e}\rangle$ as 1 . Under these assumptions, the qubit measurement process can be described by a binomial distribution. 
Given a statistical sample $X^{N}$ consisting of $N$ measurement outcomes (i.e., a statistical sample $X^{N}$ from a Bernoulli distribution with parameter $p_{\mathrm{e}}$ ), the maximum likelihood estimator of $p_{\mathrm{e}}$ (i.e., the estimated probability) is given by

$$
P_{\mathrm{e}}=\bar{X}^{N}=\frac{1}{N} \sum_{k=1}^{N} X^{k},
$$

where $X^{k}$ represents the $k$-th outcome among the $N$ measured. There are several ways to compute a confidence interval for the parameter $p_{\mathrm{e}}$. The most common result is based on the approximation of the binomial distribution with a normal distribution. This represents a good approximation in our experiments, where the number of measurements $N$ is large (typically $N \geqslant 1500$ ). In this case, it can be shown that a confidence interval for the parameter $p_{\mathrm{e}}$ is given by

$$
P_{\mathrm{e}} \pm z_{(1-\alpha / 2)} \sqrt{\frac{P_{\mathrm{e}}\left(1-P_{\mathrm{e}}\right)}{N}}=P_{\mathrm{e}} \pm z_{(1-\alpha / 2)} \tilde{\sigma}_{\mathrm{b}}
$$

where $z_{(1-\alpha / 2)}$ is the $(1-\alpha / 2)$ percentile of a standard normal distribution. For example, for a $0.95(95 \%)$ confidence interval, we set $\alpha=0.05$, so that $z_{(1-\alpha / 2)}=1.96$. When analyzing our data we approximate the percentile 1.96 with 2 , thus obtaining a slightly wider confidence interval;

(ii) Phase errors are mostly due to the phase jitter/fluctuations in the room-temperature cables and electronics used to measure the qubits. In order to quantify such errors, the following experiment was performed. First, we initialized one of the two qubits, e.g., qubit $\mathrm{Q}_{1}$, in the ground state, $\left|\mathrm{Q}_{1}\right\rangle=|\mathrm{g}\rangle$; second, we applied to $\mathrm{Q}_{1}$ a $\pi / 2$ unitary rotation about the $y$-axis, $\hat{R}_{y}^{\pi / 2}$, bringing the qubit into the state $\left|\mathrm{Q}_{1}\right\rangle=(|\mathrm{g}\rangle+|\mathrm{e}\rangle) / \sqrt{2}$. This state is characterized by the density matrix

$$
\hat{\rho}_{\mathrm{Q}_{1}}=\frac{1}{2}\left(\begin{array}{ll}
1 & 1 \\
1 & 1
\end{array}\right),
$$

which represents a "phase-sensitive" state due to the presence of nonzero off-diagonal elements, thus allowing us to measure the phase properties of our setup. In fact, if the setup (cables and electronics) were ideal, the phase $\angle\left\langle\mathrm{g}\left|\hat{\rho}_{\mathrm{Q}_{1}}\right| \mathrm{e}\right\rangle=-\angle\left\langle\mathrm{e}\left|\hat{\rho}_{\mathrm{Q}_{1}}\right| \mathrm{g}\right\rangle$ associated with the off-diagonal elements of the matrix $\hat{\rho}_{\mathrm{Q}_{1}}$ of Eq. S7 would be zero. We can thus assume that any deviation from a zero phase corresponds to a phase error; third, we performed a single-qubit quantum state tomography (QST) on $\mathrm{Q}_{1}$, making possible to measure experimentally $\hat{\rho}_{\mathrm{Q}_{1}}$. Using our typical settings for a single-qubit QST ${ }^{1}$, the time needed for each QST was approximately $8 \mathrm{~s}$; fourth, we repeated a QST measurement every $8 \mathrm{~s}$ for a total time of 40 minutes, corresponding to 300 measured density matrices; finally, we plotted $\angle\left\langle\mathrm{g}\left|\hat{\rho}_{\mathrm{Q}_{1}}\right| \mathrm{e}\right\rangle$ as a function of time. The so-obtained time trace is shown in Fig. [S1A. Besides negligible slow-varying oscillations in the time trace [independent tests have shown that these oscillations might be due to temperature changes in the room-temperature cables (data not shown)], the overall histogram associated with the trace is approximately normally distributed about a mean value of $0 \mathrm{rad}$, with standard deviation $\tilde{\sigma}_{\phi} \simeq 0.065 \mathrm{rad}$ (cf. Fig. S1B). However, we notice a general increase in the scatter of the time-trace data, as indicated by the dashed black lines in Fig. S1A. We thus divide the time trace in 10 sub-traces (time bins) with a time length of $4 \mathrm{~min}$ each, compute the standard deviation for each sub-trace, and plot the so-obtained 10 standard deviations as a function of time. The result is displayed in Fig. $\mid \mathrm{S} 1 \mathrm{C}$, where the data is overlayed with a linear fit.

The plot of Fig. S1C is useful in determining the phase errors associated with different types of two-qubit QST, as well as quantum process tomography $(\mathrm{QPT})^{2-5}$. In fact, two-qubit QST can be realized either by applying to each qubit the set of three unitary operations $\left\{\hat{I}, \hat{R}_{x}^{\pi / 2}, \hat{R}_{y}^{\pi / 2}\right\}\left(\hat{I}\right.$ is the $2 \times 2$ identity matrix, $\hat{R}_{x}^{\pi / 2}$ a $\pi / 2$ unitary rotation about the $x$-axis, and $\hat{R}_{y}^{\pi / 2}$ a $\pi / 2$ unitary rotation about the $y$-axis), which we call "tomo," or the set of six unitary operations $\left\{\hat{I}, \hat{R}_{x}^{\pi / 2}, \hat{R}_{y}^{\pi / 2}, \hat{R}_{x}^{-\pi / 2}, \hat{R}_{y}^{-\pi / 2}, \hat{R}_{x}^{\pi}\right\}\left(\hat{R}_{x}^{-\pi / 2}\right.$ is a $-\pi / 2$ unitary rotation about the $x$-axis, 
$\hat{R}_{y}^{-\pi / 2}$ a $-\pi / 2$ unitary rotation about the $y$-axis, and $\hat{R}_{x}^{\pi}$ a $\pi$ unitary rotation about the $x$-axis), which we call "octomo."

In the case of two-qubit tomo, the number of operations that must be applied to the pair of qubits is given by the permutations of the allowed set of unitary operations, $3^{2}=9$. This number multiplied by the 4 possible joint probabilities for a two-qubit system, $p_{\mathrm{gg}}, p_{\mathrm{ge}}, p_{\mathrm{eg}}$, and $p_{\mathrm{ee}}$ (where, e.g., $p_{\mathrm{ge}}$ is the probability to measure the first qubit in the ground state with the second qubit in the excited state) gives a total of 36 probabilities. In the case of octomo, the total number of probabilities is given by the permutations of 6 unitary operations for 2 qubits, $6^{2}=36$, times the 4 possible joint probabilities for a two-qubit system, for a total of 144 probabilities.

In the experiments, the maximum likelihood estimator for each of the four probabilities $p_{\mathrm{gg}}, p_{\mathrm{ge}}, p_{\mathrm{eg}}$, and $p_{\mathrm{ee}}$ is obtained from the outcome of $N$ measurements. We note that, in a joint two-qubit measurement each outcome consists of 4 numbers obtained simultaneously, where each number can be either 0 or 1 . The statistical sample consisting of $N$ two-qubit joint measurements will be hereafter defined as $X_{l m}^{N}$, with $l, m=\mathrm{g}$, e. Similarly to Eq. S5], the maximum likelihood estimator (i.e., the estimated probability) for each of the four probabilities $p_{\mathrm{gg}}, p_{\mathrm{ge}}, p_{\mathrm{eg}}$, and $p_{\mathrm{ee}}$ can thus be obtained from

$$
P_{l m}=\frac{1}{N} \sum_{k=1}^{N} X_{l m}^{k},
$$

where $X_{l m}^{k}$ represents the $k$-th outcome among the $N$ measured.

For a given $k$, the four possible $X_{l m}^{k}$, i.e., $X_{\mathrm{gg}}^{k}, X_{\mathrm{ge}}^{k}, X_{\mathrm{eg}}^{k}$, and $X_{\mathrm{ee}}^{k}$, are measured simultaneously (with $X_{\mathrm{gg}}^{k}+$ $\left.X_{\mathrm{ge}}^{k}+X_{\mathrm{eg}}^{k}+X_{\mathrm{ee}}^{k}=1\right)$. Hence, the effective number of events that has to be measured for each tomo is $36 / 4=9$, and for each octomo $144 / 4=36$.

We typically measure 2500 events per second, and repeat each measurement $N=15000$ times. As a consequence, a two-qubit tomo takes approximately $1 \mathrm{~min}$, and a two-qubit octomo approximately $4 \mathrm{~min}$.

All data displayed in Fig. 2C of the main text were obtained using tomo, while all data in Fig. 3, D and E, were obtained using octomo. All density matrices used to reconstruct the $\chi$ matrix of Fig. $3 \mathrm{~F}$ in the main text were also obtained with octomo. The standard deviation due to phase errors can be estimated in each case by looking up the fit in Fig. S1. C.

Considering for example a two-qubit octomo with $N=15000$, the statistical properties of the resulting density matrix $\hat{\rho}$ and of the corresponding metrics [fidelity $\mathcal{F}$, negativity $\mathcal{N}$, concurrence $\mathcal{C}$, and entanglement of formation $\mathcal{E}$; cf. Ref. ${ }^{[6}$ and references therein for an extensive description of these metrics] are obtained as follows:

(1) The probabilities $P_{l m}$ associated with two-qubit octomo are estimated according to Eq. S8 As explained above, this corresponds to a total of $36 \times 4=144$ estimated probabilities. To simplify the notation, we will hereafter refer to these probabilities as $P_{i}$, with $i \in\{1,2, \ldots, 144\}$;

(2) The estimated probabilities $P_{i}$ are corrected for measurement errors [cf. Refs..$^{7]}$ and ${ }^{[8}$ for our standard procedures to correct for measurement errors in the case of one and two qubits, respectively]. The corrected probabilities $P_{i}$ are stored as a $144 \times 1$ column vector;

(3) For each probability $P_{i}$, the binomial standard deviation $\tilde{\sigma}_{\mathrm{b}}$ defined in Eq. $\mathrm{S6}$ is calculated, thus obtaining, in the case of octomo, 144 different standard deviations;

(4) For each of the 144 standard deviations $\tilde{\sigma}_{\mathrm{b}}$ calculated in (3), a set of $M$ random numbers picked from a normal distribution with zero mean value and standard deviation $\tilde{\sigma}_{\mathrm{b}}$ is generated. This results in a matrix of $144 \times M$ random numbers. Typically, $M=1000$.

By summing each column of such a matrix to the column vector containing the 144 estimated probabilities $P_{i}$, we obtain a matrix of $144 \times M$ probabilities, where each column simulates the result of a different QST experiment. 


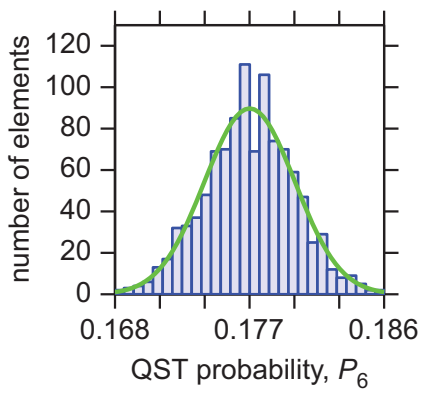

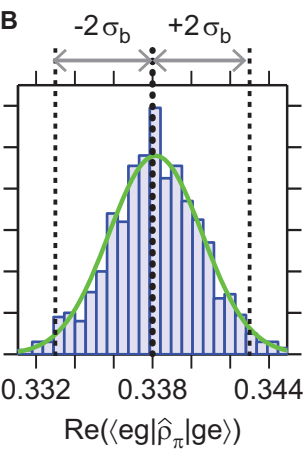

Figure S2: Confidence intervals for a density matrix and its metrics. (A) Histogram associated with the 6-th probability $P_{6}$ of the vector of probabilities $P_{i}$, plotting the number of elements among the $M$ probabilities obtained in point (4) vs. the corresponding value of the probability $P_{6}$. The data refers to the octomo for the state $\hat{\rho}_{\pi}$ of Fig. 3E in the main text. The solid green line is a fit to a normal distribution. (B) Histogram for the real part of the matrix element with mean value $\left\langle\operatorname{eg}\left|\hat{\rho}_{\pi}\right| \mathrm{ge}\right\rangle=0.338$ for the state $\hat{\rho}_{\pi}$ of Fig. 3E in the main text. The solid green line is a fit to a normal distribution. The $\pm 2 \sigma_{\mathrm{b}}$ window is indicated, where $\sigma_{\mathrm{b}}$ is one standard deviation.

For example, Fig. $\mathrm{S} 2 \mathrm{~A}$ shows the histogram associated with the 6 -th probability $P_{6}$ of the vector of probabilities $P_{i}$ in the case of the octomo for the state $\hat{\rho}_{\pi}$ of Fig. $3 \mathrm{E}$ in the main text;

(5) Each column of the $144 \times M$ matrix of probabilities obtained in point (4) is inverted by following the usual QST rules ${ }^{118}$. This allows us to find the corresponding density matrix $\hat{\rho}_{j}^{\text {unphys }}$, with $j \in\{1,2, \ldots, M\}$, thus obtaining $M$ density matrices associated with one state;

(6) Physicality constraints are enforced on each, generally unphysical, density matrix $\hat{\rho}_{j}^{\text {unphys }}$ by means of the MATLAB packages SeDuMi 1.21 and YALMIP (semidefinite programming) ${ }^{9}$. The physical constraints are such that each final - physical - density matrix $\hat{\rho}_{j}$ should have unit trace and be positive semidefinite.

In order to obtain the mean physical density matrix $\hat{\rho}$ associated with the $M$ physical density matrices $\hat{\rho}_{j}$ and the corresponding standard deviations, we calculate the mean value and standard deviation of the real and imaginary part of each matrix element for the $M$ matrices $\hat{\rho}_{j}$. The mean physical matrix $\hat{\rho}$ will thus have elements $\langle\operatorname{lm}|\hat{\rho}| p q\rangle$ (with $|l m\rangle,|p q\rangle \in \mathcal{M}_{2}$ ), each of them (real and imaginary part) characterized by a given standard deviation. Figure $\mathrm{S} 2 \mathrm{~B}$ shows the histogram for the real part of the matrix element with mean value $\left\langle\mathrm{eg}\left|\hat{\rho}_{\pi}\right| \mathrm{ge}\right\rangle=0.338$ for the state $\hat{\rho}_{\pi}$ of Fig. $3 \mathrm{E}$ in the main text. As expected, the distribution is approximately Gaussian with a 0.95 confidence interval $\pm 2 \sigma_{\mathrm{b}}= \pm 0.005$.

The knowledge of the $M$ matrices $\hat{\rho}_{j}$ also allows us to estimate the confidence intervals for the relevant metrics characterizing the state $\hat{\rho}: \mathcal{F}, \mathcal{N}, \mathcal{C}$, and $\mathcal{E}$. This can easily be accomplished by calculating the metrics for each $\hat{\rho}_{j}$, thus obtaining $M$ values for each metric, and then computing the mean value and standard deviation of the $M$ values associated with each metric.

We can follow a similar procedure to account for phase errors. We now pick two independent sets of $M$ random numbers from a normal distribution with zero mean value and standard deviation $\tilde{\sigma}_{\phi}$ (with $\tilde{\sigma}_{\phi}$ opportunely estimated from Fig. $[\mathrm{S} 1 \mathrm{C}$ depending on whether a tomo or octomo was used), thus generating two sets of $M$ phase errors, $\phi_{1}^{j}$ and $\phi_{2}^{j}$, with $j \in\{1,2, \ldots, M\}$. In order to simulate phase errors acting independently on each qubit, we apply the unitary rotation

$$
U_{j}=\left(\begin{array}{cccc}
1 & 0 & 0 & 0 \\
0 & e^{i \phi_{1}^{j}} & 0 & 0 \\
0 & 0 & e^{i \phi_{2}^{j}} & 0 \\
0 & 0 & 0 & e^{i\left(\phi_{1}^{j}+\phi_{2}^{j}\right)}
\end{array}\right)
$$


to a $4 \times 4$ measured density matrix $\hat{\rho}^{\text {meas }}$, thus obtaining the $j$-th unphysical density matrix

$$
\hat{\rho}_{j}^{\text {unphys }}=U_{j} \hat{\rho}^{\text {meas }} U_{j}^{\dagger} \text {. }
$$

We can then proceed as in step (6) above and obtain a mean physical density matrix $\hat{\rho}$ and its statistical properties, as in the case of binomial-type errors. This allows us also to find the metrics associated with $\hat{\rho}$ and their statistical properties. Notice that the unitary transformation of Eq. S9 simulates random rotations along the $z$-axis of both qubit $Q_{1}$ and qubit $Q_{2}$.

The total mean physical density matrix is finally obtained by averaging the mean physical density matrix obtained in the case of binomial-type errors and the matrix obtained in the case of phase errors. The same applies to the mean values of all metrics. The corresponding standard deviations are found by summing in quadrature the values obtained in the case of binomial-type and phase errors. For example, the numerical value with confidence interval of each element of the density matrices in Fig. $2 \mathrm{C}$ of the main text were obtained following this procedure. These numbers are reported in Table $\mathrm{S} 1$.

Incidentally, we found that phase errors do not add any significant contribution to the confidence intervals of the density matrix elements and of their metrics.

Notice that, the reason why we decided to simulate the statistical properties of the data in Fig. 2 of the main text is because we only had 2 independent measurements of these data. Such a statistical ensemble is obviously insufficient to obtain reliable confidence intervals, which, thus, needed to be simulated.

\section{Experimental estimation of statistical errors}

In the case of the density matrices in Fig. 3E and of the $\chi_{\mathrm{m}}^{\mathrm{p}}$ matrix of the quantum Fourier transform in Fig. 3F of the main text we had ensembles of independent measurements large enough to allow the confidence intervals estimation directly from the data.

In particular, the density matrix $\hat{\rho}_{0.28}$ in the left panel of Fig. $3 \mathrm{E}$ is the average of a statistical ensemble of $M=10$ independent measurements, and the density matrices $\hat{\rho}_{\pi / 2}$ and $\hat{\rho}_{\pi}$ in the center and right panels of Fig. 3E, respectively, are the average of an ensemble of $M=6$ independent measurements. The standard deviation of each matrix element (real and imaginary part) as well as the mean value and standard deviation of all metrics can easily be estimated from such statistical ensembles.

Finally, the matrix $\chi_{\mathrm{m}}^{\mathrm{p}}$ of Fig. $3 \mathrm{~F}$ is the average of an ensemble of 15 independent measurements. This allows us to estimate the mean value and standard deviation of the process fidelity $\mathcal{F}_{\chi}$ associated with the quantum Fourier transform (cf. main text).

\section{Statistical errors of fitted parameters}

The confidence intervals associated with the quantum phase tomography data shown in Fig. 4, C and F, of the main text are dominated by the statistical errors of the coefficients fitted from the data in Fig. 4, B and E, of the main text. In particular, the coefficient of interest is the phase of each curve in Fig. 4, B and E (or, more in general, of each curve in Fig. S12, C and D).

We remind that the error vector associated with the vector of coefficients fitted to a curve is given by the square root of the vector $\vec{S}$ of the diagonal elements from the estimated covariance matrix of the coefficient estimates, $\left(\vec{X}^{T} \vec{X}\right)^{-1}\langle s\rangle^{2}$. Here, $\vec{X}$ is the Jacobian of the fitted values with respect to the coefficients, $\vec{X}^{T}$ is the transpose of $\vec{X}$, and $\langle s\rangle^{2}$ is the mean squared error. This procedure allows us to estimate the errors associated with the fitted phases. These errors propagate through the quantum tomography process (cf. section on "Quantum phase tomography" at the end of these Methods), finally turning into the confidence intervals reported in Fig. 4, C and $\mathrm{F}$, of the main text. 


\section{Definition of the qubit reference frame}

In this section, we briefly explain the concepts of reference frame and reference clock rate associated with a qubit. These concepts will be useful in understanding the dynamic phases acquired by the qubits when programming the quantum von Neumann architecture as well as the sequences used to tune up the CZ- $\phi$ gates and the XOR and M gate.

In the two-level approximation $\frac{10}{10}$, the Hamiltonian of a phase qubit can be written as

$$
\widehat{H}_{\mathrm{Q}}=h \frac{f_{\mathrm{Q}}(z)}{2} \hat{\sigma}_{z},
$$

with ground state $|\mathrm{g}\rangle$ and excited state $|\mathrm{e}\rangle$, and eigenenergies $E_{\mathrm{g}}$ and $E_{\mathrm{e}}$, respectively. In Eq. $\mathrm{S} 11, f_{\mathrm{Q}}(z) \equiv$ $\Delta E_{\mathrm{eg}} / h=\left(E_{\mathrm{e}}-E_{\mathrm{g}}\right) / h$ represents the qubit transition frequency, which can be tuned by means of $\mathrm{z}$-pulses with amplitude $z$, and $\hat{\sigma}_{z}$ is the usual spin $1 / 2$ Pauli operator. At the beginning of a CZ- $\phi$ gate, each qubit is initialized in $|\mathrm{g}\rangle$ at the so-called idle point, which corresponds to a z-pulse amplitude $z=0$. The qubit transition frequency at the idle point is thus given by $f_{\mathrm{Q}}(z=0) \equiv f_{\mathrm{Q}}^{0}$.

In order to prepare a qubit in the excited state $|\mathrm{e}\rangle$ or in a linear superposition $|\mathrm{g}\rangle+|\mathrm{e}\rangle$, the qubit has to be driven by a microwave pulse. The Hamiltonian governing the interaction between the qubit and the microwave driving is given by 11

$$
\widehat{H}_{\mathrm{D}}=h \Omega_{\mathrm{D}}(\tau) \hat{\sigma}_{y} \sin \left(2 \pi f_{\mathrm{D}} \tau+\phi_{\text {delay }}\right),
$$

where $\Omega_{\mathrm{D}}(\tau)$ is the time-dependent driving amplitude expressed in unit hertz, $f_{\mathrm{D}}$ the driving frequency, $\hat{\sigma}_{y}$ the usual spin $1 / 2$ Pauli operator, $\tau$ the time, and $\phi_{\text {delay }}$ an arbitrary phase delay. By calibrating the microwave pulse such that the phase delay $\phi_{\text {delay }}=\pi / 2$, we can rewrite the driving Hamiltonian as

$$
\widehat{H}_{\mathrm{D}}=h \Omega_{\mathrm{D}}(\tau) \hat{\sigma}_{y} \cos \left(2 \pi f_{\mathrm{D}} \tau\right)
$$

By combining the qubit Hamiltonian of Eq. S11 and the driving Hamiltonian of Eq. S13, we obtain the total Hamiltonian of the driven system, $\widehat{H}_{\mathrm{QD}}=\widehat{H}_{\mathrm{Q}}+H_{\mathrm{D}}$.

In our experiments the driving frequency $f_{\mathrm{D}}$ is a fixed parameter that is set to be equal to the qubit transition frequency at the idle point ${ }^{12}$,

$$
f_{\mathrm{D}}=f_{\mathrm{Q}}^{0} \text {. }
$$

For a given qubit, the microwave driving represents the reference frame associated with that qubit, with reference clock rate given by $f_{\mathrm{Q}}^{0}$. Defining the detuning between the z-dependent qubit transition frequency $f_{\mathrm{Q}}(z)$ and the reference clock rate $f_{\mathrm{Q}}^{0}$ as $\Delta(z) \equiv f_{\mathrm{Q}}(z)-f_{\mathrm{Q}}^{0}$, the qubit-driving Hamiltonian $\widehat{H}_{\mathrm{QD}}$ can be expressed in the uniformly rotating reference frame by applying the unitary rotation $\widehat{D}=e^{+i 2 \pi f_{\mathrm{Q}}^{0} \tau \hat{\sigma}_{z} / 213}$. The rotated Hamiltonian is thus given by

$$
\begin{aligned}
\widehat{\widetilde{H}}_{\mathrm{QD}} & =\widehat{D} \widehat{H}_{\mathrm{QD}} \widehat{D}^{\dagger}-i \hbar \widehat{D} \frac{d}{d \tau} \widehat{D}^{\dagger} \\
& \approx-h \frac{\Delta(z)}{2} \hat{\sigma}_{z}+h \frac{\Omega_{\mathrm{D}}(\tau)}{2} \hat{\sigma}_{y},
\end{aligned}
$$

where the counter-rotating terms have been already neglected. The dynamics associated with the pulse sequences used to tune up the $\mathrm{CZ}-\phi$ gates and the XOR and M gate can be understood by following the time-evolution of the Hamiltonian of Eq. $\mathrm{S} 14$ In particular, the Hamiltonian $\widehat{\widetilde{H}}_{\mathrm{QD}}$ describes the dynamic phases acquired by the qubits when they are brought outside their reference frame (i.e., qubit rotations about the $z$-axis). As it will appear clear when describing the tune-up sequences of the CZ- $\phi$ gates and of the XOR and M gate, in the experiments we always compensate for such dynamic phases. 


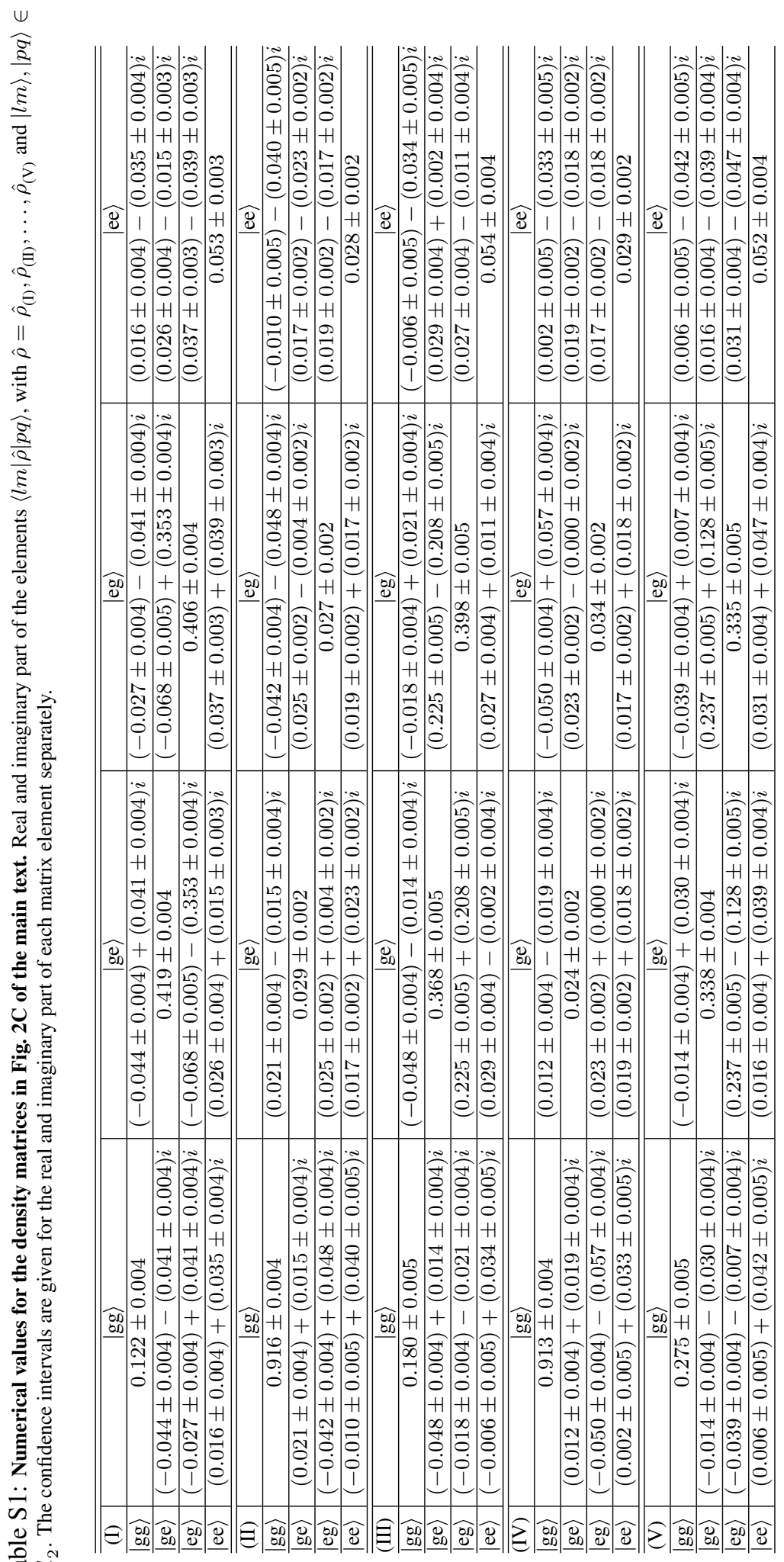




\section{Programming the quantum von Neumann architecture}

The phase difference between the off-diagonal elements of the density matrices shown in Fig. $2 \mathrm{C}$ of the main text (red arrows) are due to the qubits being brought outside their reference frame during the pulse sequence in Fig. 2A (the qubits acquire dynamic phases), and to the angle accumulated by the microwave signal used to excite the qubits. The pulse sequence was calibrated such that the first density matrix $\hat{\rho}_{(\mathrm{I})}$ has purely imaginary off-diagonal elements [cf. grey and overlayed red arrows in Fig. 2C (I) of the main text]. We can thus calculate the angles of the density matrices $\hat{\rho}_{(\mathrm{III})}$ and $\hat{\rho}_{(\mathrm{V})}$ by knowing the time duration of the various steps in the sequence and the corresponding qubit detunings (obtained from independent measurements), as shown by the grey arrows in the matrices of Fig. 2C, (III) and (V). As expected, the experimentally measured red arrows overlay the calculated grey arrows with high accuracy. We will later show a pulse method that allows us to compensate for dynamic phases during the experiment, rather than calibrating the phases a posteriori as in Fig. $2 \mathrm{C}$. Such a compensation pulse method was used to implement the quantum Fourier transform and the XOR and M gate.

The numerical values of all elements (real and imaginary part) of each density matrix in Fig. $2 \mathrm{C}$ of the main text are reported in Table $\mathrm{S} 1$. The confidence interval for the real and imaginary part of each complex number is also indicated. The confidence intervals correspond to two standard deviations (95\% confidence interval), where the standard deviations were calculated as explained in the section on "Statistical errors" of these Methods. 


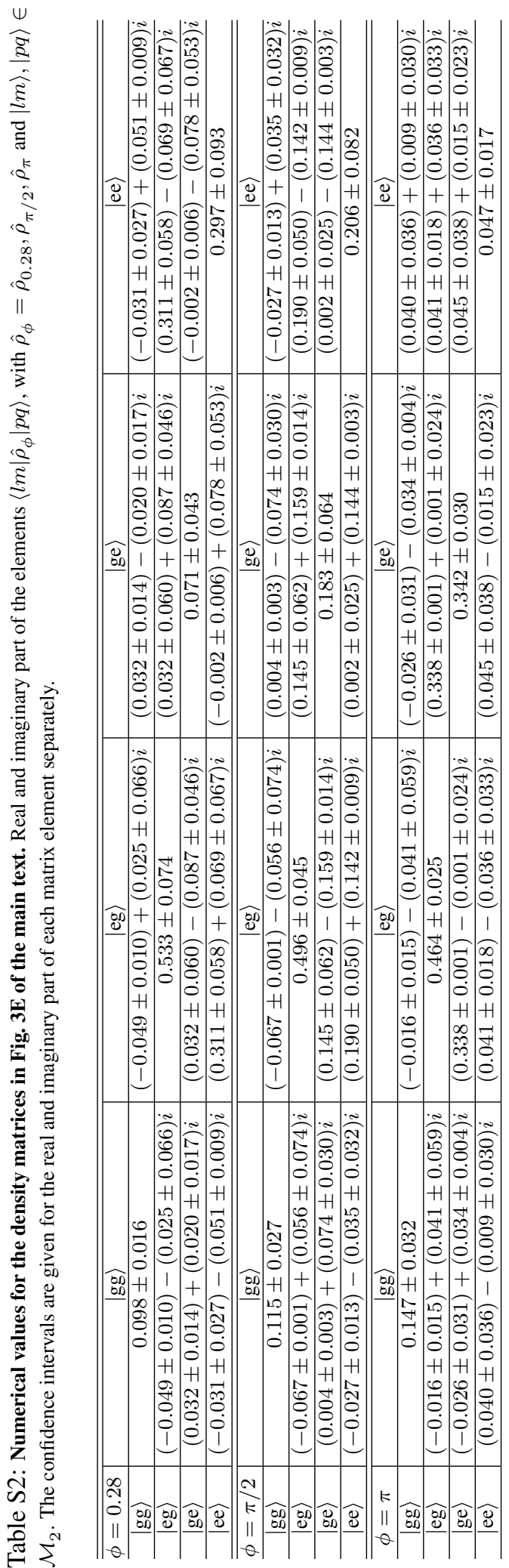



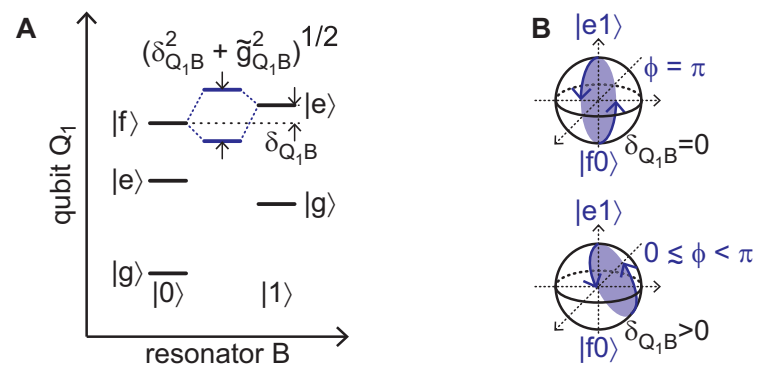

Figure S3: The CZ- $\phi$ gate energy diagram. (A) Energy diagram for target qubit $\mathrm{Q}_{1}$ coupled to bus resonator $\mathrm{B}$, with $\mathrm{Q}_{1}$ 's eigenstates indicated by $|\mathrm{g}\rangle,|\mathrm{e}\rangle$, and $|\mathrm{f}\rangle$, and B's eigenstates indicated by $|0\rangle$ and $|1\rangle$. In general, states $|\mathrm{f} 0\rangle$ and $|\mathrm{e} 1\rangle$ are detuned by a quantity $\delta_{\mathrm{Q}_{1} \mathrm{~B}}$. Thus, their effective Rabi frequency is given by $\left(\delta_{\mathrm{Q}_{1} \mathrm{~B}}^{2}+\tilde{g}_{\mathrm{Q}_{1} \mathrm{~B}}^{2}\right)^{1 / 2}$. (B) Bloch sphere interpretation of the $|\mathrm{f} 0\rangle-|\mathrm{e} 1\rangle$ interaction. When $\delta_{\mathrm{Q}_{1} \mathrm{~B}}=0$, $\mathrm{Q}_{1}$ acquires a phase $\phi=\pi$ (Top). When $\delta_{\mathrm{Q}_{1} \mathrm{~B}}>0, \mathrm{Q}_{1}$ acquires a phase $0 \lesssim \phi<\pi$ (Bottom).

\section{The quantum Fourier transform}

The numerical values of all elements (real and imaginary part) of each density matrix in Fig. 3E of the main text are reported in Table S2. The $95 \%$ confidence interval for the real and imaginary part of each complex number is also indicated. The standard deviations were calculated as explained in the section on "Statistical errors" of these Methods.

As shown in the main text, the CZ- $\phi$ gate is a fundamental element for the implementation of the quantum Fourier transform. In the rest of this section, we derive the analytical expression for the phase $\phi$ of a CZ- $\phi$ gate by diagonalizing the effective Hamiltonian of the $\mathrm{Q}_{1}-\mathrm{B}-\mathrm{Q}_{2}$ system and calculating its time evolution. We subsequently describe the experimental pulse sequences required to tune up the CZ- $\phi$ gate and show three examples of Ramsey experiments used to measure the gate phase $\phi$, when $\phi=0.01, \phi=\pi / 2$, and $\phi=\pi$. Finally, we discuss the origin of systematic errors in the measurement of the phase $\phi$, showing that the global phase shift in the curve of Fig. $3 \mathrm{C}$ of the main text is due to a drift of the qubit operation point.

\section{Analytical expression of the phase $\phi$ of a CZ- $\phi$ gate}

The CZ- $\phi$ gate demonstrated in the main text makes use of a bus resonator B that mediates the interaction between qubit $\mathrm{Q}_{1}$ and $\mathrm{Q}_{2}$. During the $\mathrm{CZ}-\phi$ gate qubit $\mathrm{Q}_{1}$ is used as a qutrit, where the third eigenstate $|\mathrm{f}\rangle$ plays an active role in the implementation of the gate. Qubit $\mathrm{Q}_{1}$ represents the gate target and qubit $\mathrm{Q}_{2}$ the gate control. The energy diagram of the $\mathrm{Q}_{1}-\mathrm{B}$ coupled system is displayed in Fig. $\mathrm{S} 3 \mathrm{~A}$. The coupled system consists of the states $|\mathrm{g}\rangle,|\mathrm{e}\rangle$, and $|\mathrm{f}\rangle$ of the target qubit $\mathrm{Q}_{1}$, and of the states $|0\rangle$ and $|1\rangle$ of the bus resonator $\mathrm{B}$. In the rotating frame of resonator $\mathrm{B}$ and using the rotating-wave approximation, the system effective Hamiltonian can be written as

$$
\widehat{H}_{\mathrm{eff}}=h \Delta|\mathrm{e} 1\rangle\left\langle\mathrm{e} 1\left|+h\left(\Delta+\delta_{\mathrm{Q}_{1} \mathrm{~B}}\right)\right| \mathrm{f} 0\right\rangle\langle\mathrm{f} 0|+h \frac{\tilde{g}_{\mathrm{Q}_{1} \mathrm{~B}}}{2}(|\mathrm{e} 1\rangle\langle\mathrm{f} 0|+| \mathrm{f} 0\rangle\langle\mathrm{e} 1|),
$$

where $\Delta$ represents the frequency detuning of $\mathrm{Q}_{1}$ with respect to the reference frame, $\delta_{\mathrm{Q}_{1} \mathrm{~B}}$ the frequency detuning between states $|\mathrm{f} 0\rangle$ and $|\mathrm{e} 1\rangle$, and $\tilde{g}_{\mathrm{Q}_{1} \mathrm{~B}}$ their on-resonance coupling. As already mentioned after Eq. S14. in the experiments we compensate the rotation about the $z$-axis of $\mathrm{Q}_{1}$ associated with the detuning $\Delta$. As a consequence, in Eq. $\mathrm{S} 15$ we can set $\Delta=0$ and rewrite the system effective Hamiltonian as

$$
\widehat{H}_{\mathrm{eff}}=h \delta_{\mathrm{Q}_{1} \mathrm{~B}}|\mathrm{f} 0\rangle\langle\mathrm{f} 0|+h \frac{\tilde{g}_{\mathrm{Q}_{1} \mathrm{~B}}}{2}(|\mathrm{e} 1\rangle\langle\mathrm{f} 0|+| \mathrm{f} 0\rangle\langle\mathrm{e} 1|) .
$$


The diagonalization of the Hamiltonian of Eq. S16 gives the eigenstates

$$
\begin{aligned}
& \left|\mathrm{Q}_{1} \mathrm{~B}\right\rangle_{-}=\frac{\sqrt{1+\delta_{\mathrm{Q}_{1} \mathrm{~B}} / \sqrt{\delta_{\mathrm{Q}_{1} \mathrm{~B}}^{2}+\tilde{g}_{\mathrm{Q}_{1} \mathrm{~B}}^{2}}}|\mathrm{e} 1\rangle-\sqrt{1-\delta_{\mathrm{Q}_{1} \mathrm{~B}} / \sqrt{\delta_{\mathrm{Q}_{1} \mathrm{~B}}^{2}+\tilde{g}_{\mathrm{Q}_{1} \mathrm{~B}}^{2}}}|\mathrm{f} 0\rangle}{\sqrt{2}}, \\
& \left|\mathrm{Q}_{1} \mathrm{~B}\right\rangle_{+}=\frac{\sqrt{1-\delta_{\mathrm{Q}_{1} \mathrm{~B}} / \sqrt{\delta_{\mathrm{Q}_{1} \mathrm{~B}}^{2}+\tilde{g}_{\mathrm{Q}_{1} \mathrm{~B}}^{2}}}|\mathrm{e} 1\rangle+\sqrt{1+\delta_{\mathrm{Q}_{1} \mathrm{~B}} / \sqrt{\delta_{\mathrm{Q}_{1} \mathrm{~B}}^{2}+\tilde{g}_{\mathrm{Q}_{1} \mathrm{~B}}^{2}}}|\mathrm{f} 0\rangle}{\sqrt{2}},
\end{aligned}
$$

with eigenenergies

$$
\begin{aligned}
& E_{-}=\frac{\delta_{\mathrm{Q}_{1} \mathrm{~B}}-\sqrt{\delta_{\mathrm{Q}_{1} \mathrm{~B}}^{2}+\tilde{g}_{\mathrm{Q}_{1} \mathrm{~B}}^{2}}}{2} \\
& E_{+}=\frac{\delta_{\mathrm{Q}_{1} \mathrm{~B}}+\sqrt{\delta_{\mathrm{Q}_{1} \mathrm{~B}}^{2}+\tilde{g}_{\mathrm{Q}_{1} \mathrm{~B}}^{2}}}{2}
\end{aligned}
$$

respectively.

Given the initial state $\left|\mathrm{Q}_{1} \mathrm{~B}\right\rangle_{0}=|\mathrm{e} 1\rangle$, after a time $\tau$ the evolution $\widehat{U}(\tau)=\exp \left(-i \widehat{H}_{\mathrm{eff}} \tau / \hbar\right)$ of the effective Hamiltonian of Eq. S16 acting on $\left|\mathrm{Q}_{1} \mathrm{~B}\right\rangle_{0}$ results in the state

$$
\begin{aligned}
\widehat{U}(\tau)\left|\mathrm{Q}_{1} \mathrm{~B}\right\rangle_{0}= & \frac{1}{\sqrt{2}}\left(\sqrt{1+\delta_{\mathrm{Q}_{1} \mathrm{~B}} / \sqrt{\delta_{\mathrm{Q}_{1} \mathrm{~B}}^{2}+\tilde{g}_{\mathrm{Q}_{1} \mathrm{~B}}^{2}}}\left|\mathrm{Q}_{1} \mathrm{~B}\right\rangle_{-} e^{-i E_{-} \tau}\right. \\
& \left.+\sqrt{1-\delta_{\mathrm{Q}_{1} \mathrm{~B}} / \sqrt{\delta_{\mathrm{Q}_{1} \mathrm{~B}}^{2}+\tilde{g}_{\mathrm{Q}_{1} \mathrm{~B}}^{2}}}\left|\mathrm{Q}_{1} \mathrm{~B}\right\rangle_{+} e^{-i E_{+} \tau}\right) .
\end{aligned}
$$

For a time corresponding to a full $2 \pi$-rotation between the states $|\mathrm{f} 0\rangle$ and $|\mathrm{e} 1\rangle, \tau=1 /\left(\delta_{\mathrm{Q}_{1} \mathrm{~B}}^{2}+\tilde{g}_{\mathrm{Q}_{1} \mathrm{~B}}^{2}\right)^{1 / 2}$, the $\mathrm{Q}_{1}-\mathrm{B}$ coupled system is in the state

$$
\begin{aligned}
\left|\mathrm{Q}_{1} \mathrm{~B}\right\rangle= & \frac{1}{\sqrt{2}}\left[\sqrt{1+\delta_{\mathrm{Q}_{1} \mathrm{~B}} / \sqrt{\delta_{\mathrm{Q}_{1} \mathrm{~B}}^{2}+\tilde{g}_{\mathrm{Q}_{1} \mathrm{~B}}^{2}}}\left|\mathrm{Q}_{1} \mathrm{~B}\right\rangle_{-} e^{-i\left(\delta_{\mathrm{Q}_{1} \mathrm{~B}}^{2} \tau / 2-\pi\right)}\right. \\
& \left.+\sqrt{1-\delta_{\mathrm{Q}_{1} \mathrm{~B}} / \sqrt{\delta_{\mathrm{Q}_{1} \mathrm{~B}}^{2}+\tilde{g}_{\mathrm{Q}_{1} \mathrm{~B}}^{2}}}\left|\mathrm{Q}_{1} \mathrm{~B}\right\rangle_{+} e^{-i\left(\delta_{\mathrm{Q}_{1} \mathrm{~B}}^{2} \tau / 2+\pi\right)}\right]
\end{aligned}
$$

which, for simplicity, can be rewritten as

$$
\left|\mathrm{Q}_{1} \mathrm{~B}\right\rangle=e^{i \phi}|\mathrm{e} 1\rangle
$$

where the phase $\phi$ is defined as

$$
\phi \equiv \pi-\pi \frac{\delta_{\mathrm{Q}_{1} \mathrm{~B}}}{\sqrt{\delta_{\mathrm{Q}_{1} \mathrm{~B}}^{2}+\tilde{g}_{\mathrm{Q}_{1} \mathrm{~B}}^{2}}} .
$$

Figure $\mathrm{S} 3 \mathrm{~B}$ depicts the Bloch sphere of the $\mathrm{Q}_{1}-\mathrm{B}$ coupled system for the states $|\mathrm{e} 1\rangle$ and $|\mathrm{f} 0\rangle$, showing that the interaction dynamics between the two states always starts and ends at the same pole of the sphere. It is during this interaction that the phase $\phi$ of Eq. $\mathrm{S} 24$ is acquired by the target qubit $\mathrm{Q}_{1}$. In particular, when $\delta_{\mathrm{Q}_{1} \mathrm{~B}}=0$ we obtain $\phi=\pi$, while for $\delta_{\mathrm{Q}_{1} \mathrm{~B}}>0$ we obtain all phases $0 \lesssim \phi<\pi$.

An effective Hamiltonian similar to that of Eq. $\mathrm{S} 16$ governs the interaction dynamics for the $\mathrm{Q}_{2}-\mathrm{B}$ system. Hence, the interaction between the states of both the $Q_{1}-B$ and $Q_{2}-B$ systems always starts and ends at the same pole of the coupling Bloch sphere. This has the important consequence that the CZ- $\phi$ gates used here are insensi- 

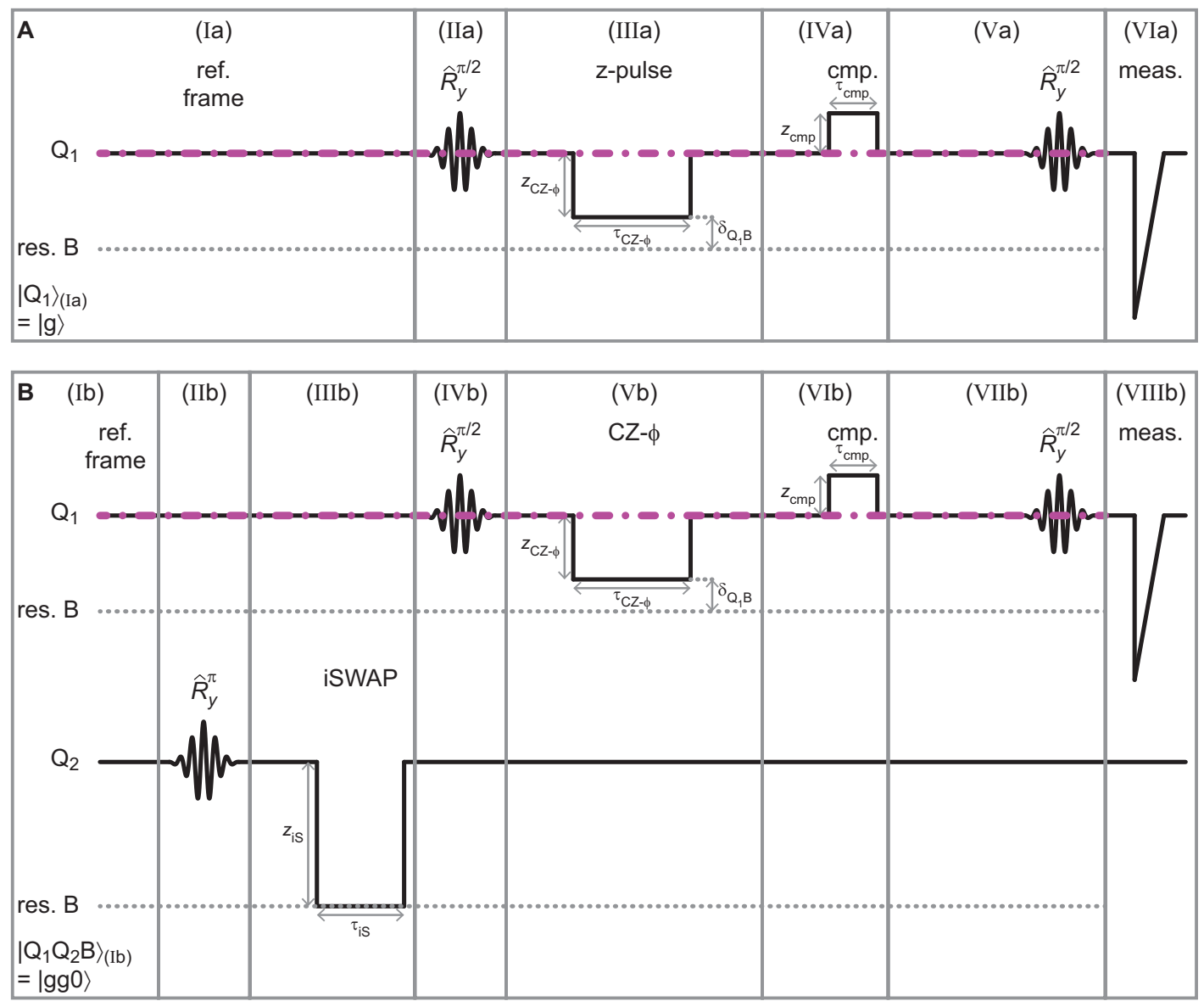

Figure S4: Dynamic phase compensation for qubit $\mathbf{Q}_{1}$. (A) Sequence without pulses on qubit $\mathrm{Q}_{2}$. Sequence steps: (Ia), $\mathrm{Q}_{1}$ is initialized in state $\left|\mathrm{Q}_{1}\right\rangle_{(\mathrm{Ia})}=|\mathrm{g}\rangle$ at the idle point. The reference frame of $\mathrm{Q}_{1}$ is indicated by a dash-dot magenta line. Resonator $\mathrm{B}$, which is indicated by a dotted grey line, is in the vacuum state $|0\rangle$; (IIa), rotation $\hat{R}_{y}^{\pi / 2}$ on $\mathrm{Q}_{1}$. The Gaussian pulse has a FWHM $\tau_{\text {FWHM }}=8 \mathrm{~ns}$; (IIIa), z-pulse on $\mathrm{Q}_{1}$ with amplitude $z_{\mathrm{CZ}-\phi}$ and length $\tau_{\mathrm{CZ}-\phi} ;$ (IVa), compensation pulse on $\mathrm{Q}_{1}$ with amplitude $z_{\text {cmp }}$ and length $\tau_{\text {cmp }}$; (Va), rotation $\hat{R}_{y}^{\pi / 2}$ on $\mathrm{Q}_{1}$; (VIa), measurement pulse on $\mathrm{Q}_{1}$. (B) Sequence with pulses on qubit $\mathrm{Q}_{2}$. Sequence steps: (Ib), the $\mathrm{Q}_{1}-\mathrm{Q}_{2}-\mathrm{B}$ system is initialized in state $\left|\mathrm{Q}_{1} \mathrm{Q}_{2} \mathrm{~B}\right\rangle_{(\mathrm{Ib})}=|\mathrm{gg} 0\rangle$, with both qubits at the idle point; (IIb), rotation $\hat{R}_{y}^{\pi}$ on $\mathrm{Q}_{2}$. The Gaussian pulse, in this case, has a FWHM $\tau_{\mathrm{FWHM}}=7 \mathrm{~ns}$; (IIIb), iSWAP between $\mathrm{Q}_{2}$ and B with amplitude $z_{\mathrm{iS}}$ and length $\tau_{\mathrm{iS}}=24.97 \mathrm{~ns}$; (IVb)-(VIIIb), same as in steps (IIa)-(VIa) of $\mathrm{A}$. Note that, in step $(\mathrm{Vb})$ the z-pulse on $\mathrm{Q}_{1}$ generates the phase $\phi$ of the CZ- $\phi$ gate.

tive to the relative phases of qubits $\mathrm{Q}_{1}$ and $\mathrm{Q}_{2}$ when they are brought into resonance via resonator $\mathrm{B}$. This feature allows us to use independent reference frames with incommensurate frequencies (and, hence, no special phase relationship) for each qubit, thus making possible to tune up each qubit with a separate calibration sequence. 


\section{CZ- $\phi$ gate tuneup}

Figure S4, A and B, shows the two sequences used to calibrate the pulses applied to qubit $\mathrm{Q}_{1}$ during the $\mathrm{CZ}-\phi$ gate operation. We note that in the calibration sequence of Fig. $\mathrm{S} 4 \mathrm{~B}$ a series of pulses is applied to qubit $\mathrm{Q}_{1}$ as well as to qubit $\mathrm{Q}_{2}$. We will show that by comparing the results obtained from the calibration of $\mathrm{Q}_{1}$ without pulsing $\mathrm{Q}_{2}$ (Fig. S4A and Fig. S5 A) with those obtained by pulsing $\mathrm{Q}_{2}$ (Fig. S4B and Fig. S5B) it is possible to measure the phase $\phi$ associated with the CZ- $\phi$ gate (cf. also main text and Fig. 3C of the main text).

Before delving into the analysis of the calibration sequences, we note that the idle point of qubits $\mathrm{Q}_{1}$ and $\mathrm{Q}_{2}$ was set at a different position depending on the experiment. For the experiments of Fig. 2, $\mathrm{C}$ to $\mathrm{E}$, in the main text, the idle point was set in between the memory and bus resonator for both qubits. This is also the case for the swap spectroscopies shown in Fig. 1B of the main text. For all the other experiments, e.g., those described in this section, the idle point was set above the bus resonator for both qubits.

Consistently with the vertical axis in Fig. 1B of the main text, a z-pulse in the upward direction, which increases the qubit transition frequency, always corresponds to a negative z-pulse amplitude with respect to the qubit idle point. The opposite applies to the case of a z-pulse in the downward direction.

The first calibration sequence for qubit $\mathrm{Q}_{1}$, which is shown in Fig. S4A, comprises the following steps:

(Ia) Qubit $Q_{1}$ is initialized in the ground state $\left|Q_{1}\right\rangle_{(\mathrm{Ia})}=|\mathrm{g}\rangle$ at the idle point, setting the qubit reference frame with reference clock rate $f_{\mathrm{Q}_{1}}^{0}$. In Fig. $\mathrm{S} 4 \mathrm{~A}$, the reference frame is indicated by the dash-dot magenta line. During the entire calibration sequence, the bus resonator B is maintained in the vacuum state $|0\rangle$. Nevertheless, in Fig. S4A we indicate the presence of resonator B by a dotted grey line, which helps visualizing the frequency detuning between qubit and resonator;

(IIa) Keeping the qubit detuning $\Delta=0$, a Gaussian microwave pulse with full width at half maximum (FWHM) $\tau_{\text {FWHM }}$ is applied to $\mathrm{Q}_{1}$. The amplitude of the pulse is chosen such that $2 \pi \Omega_{\mathrm{D}} \tau=\pi / 2$. In this case, the time evolution of the Hamiltonian of Eq. $\mathrm{S} 14$ yields a $\pi / 2$ unitary rotation about the $y$-axis $\frac{14}{R_{y}} \hat{R}^{\pi / 2}$, which brings the qubit into the new state $\left|\mathrm{Q}_{1}\right\rangle_{\text {(IIa) }}=(|\mathrm{g}\rangle+|\mathrm{e}\rangle) / \sqrt{2}$;

(IIIa) A z-pulse with amplitude $z_{\mathrm{CZ}-\phi}$, corresponding to a qubit frequency detuning $\Delta\left(z_{\mathrm{CZ}-\phi}\right)$ from the reference frame, brings the $|\mathrm{e}\rangle \leftrightarrow|\mathrm{f}\rangle$ transition of $\mathrm{Q}_{1}$ on or near resonance with B for a time $\tau_{\mathrm{CZ}-\phi}$. In general, the z-pulse can be adjusted so that the $|\mathrm{e}\rangle \leftrightarrow|\mathrm{f}\rangle$ qubit transition is detuned by a frequency $\delta_{\mathrm{Q}_{1} \mathrm{~B}}$ from resonator B (cf. Fig. S4A, and also the section on the "Analytical expression for the phase $\phi$ of a CZ- $\phi$ gate" in these Methods, and the main text). During the z-pulse, since the $|e 1\rangle-|f 0\rangle$ transition of the $\mathrm{Q}_{1}$-B coupled system is on or near resonance, the system remains always in the state $\left.\left|Q_{1} B\right\rangle=(|g\rangle+|e\rangle) / \sqrt{2}\right\rangle \otimes|0\rangle$. This represents a dark state of the time evolution of the coupled system, yielding no swaps between $\mathrm{Q}_{1}$ and $\mathrm{B}$, as opposed to the bright state $\left.\left|\mathrm{Q}_{1} \mathrm{~B}\right\rangle=(|\mathrm{g}\rangle+|\mathrm{e}\rangle) / \sqrt{2}\right\rangle \otimes|1\rangle$. Depending on the z-pulse amplitude $z_{\mathrm{CZ}-\phi}$ and, hence, on the detuning $\delta_{\mathrm{Q}_{1} \mathrm{~B}}$, the z-pulse length is chosen such that $\tau_{\mathrm{CZ}-\phi}=1 /\left(\delta_{\mathrm{Q}_{1} \mathrm{~B}}^{2}+\tilde{g}_{\mathrm{Q}_{1} \mathrm{~B}}^{2}\right)^{1 / 2}$ (cf. section on the "Analytical expression for the phase $\phi$ of a CZ- $\phi$ gate" in these Methods). The z-pulse $z_{\mathrm{CZ}-\phi}$ moves $\mathrm{Q}_{1}$ outside its reference frame. As a consequence, the time evolution of Eq. $\mathrm{S} 14$ acting on the state $\left|\mathrm{Q}_{1}\right\rangle_{\text {(IIa) }}$ gives the state $\left|\mathrm{Q}_{1}\right\rangle_{(\mathrm{III})}=\left(|\mathrm{g}\rangle e^{-i \phi_{\mathrm{dyn}}}+|\mathrm{e}\rangle e^{+i \phi_{\mathrm{dyn}}}\right) / \sqrt{2}$, where $\phi_{\mathrm{dyn}} \equiv-\Delta\left(z_{\mathrm{CZ}-\phi}\right) \tau_{\mathrm{CZ}-\phi} / 2$. This means that during the z-pulse $\mathrm{Q}_{1}$ acquires an unwanted dynamic phase $\phi_{\mathrm{dyn}}$;

(IVa) For the correct operation of the CZ- $\phi$ gate, the dynamic phase $\phi_{\text {dyn }}$ must be compensated. This can be realized by applying a compensation z-pulse to $\mathrm{Q}_{1}$, with fixed length $\tau_{\text {cmp }}$ and variable amplitude $z_{\text {cmp }}$. In order to avoid crossing the resonance with $\mathrm{B}$, the amplitude of the compensation pulse is swept in the opposite direction as compared to the z-pulse $z_{\mathrm{CZ}-\phi}$ (cf. Fig. S4A). In this case, the time evolution of $\widehat{\widetilde{H}}_{\mathrm{QD}}$ acting on $\left|\mathrm{Q}_{1}\right\rangle_{\text {(IIIa) }}$ yields the state $\left|\mathrm{Q}_{1}\right\rangle_{(\mathrm{IVa})}=\left(|\mathrm{g}\rangle e^{-i \phi_{\mathrm{a}} / 2}+|\mathrm{e}\rangle e^{+i \phi_{\mathrm{a}} / 2}\right) / \sqrt{2}$, where $\phi_{\mathrm{a}} / 2 \equiv \phi_{\text {dyn }}-$ $\Delta\left(z_{\mathrm{cmp}}\right) \tau_{\mathrm{cmp}} / 2$ 

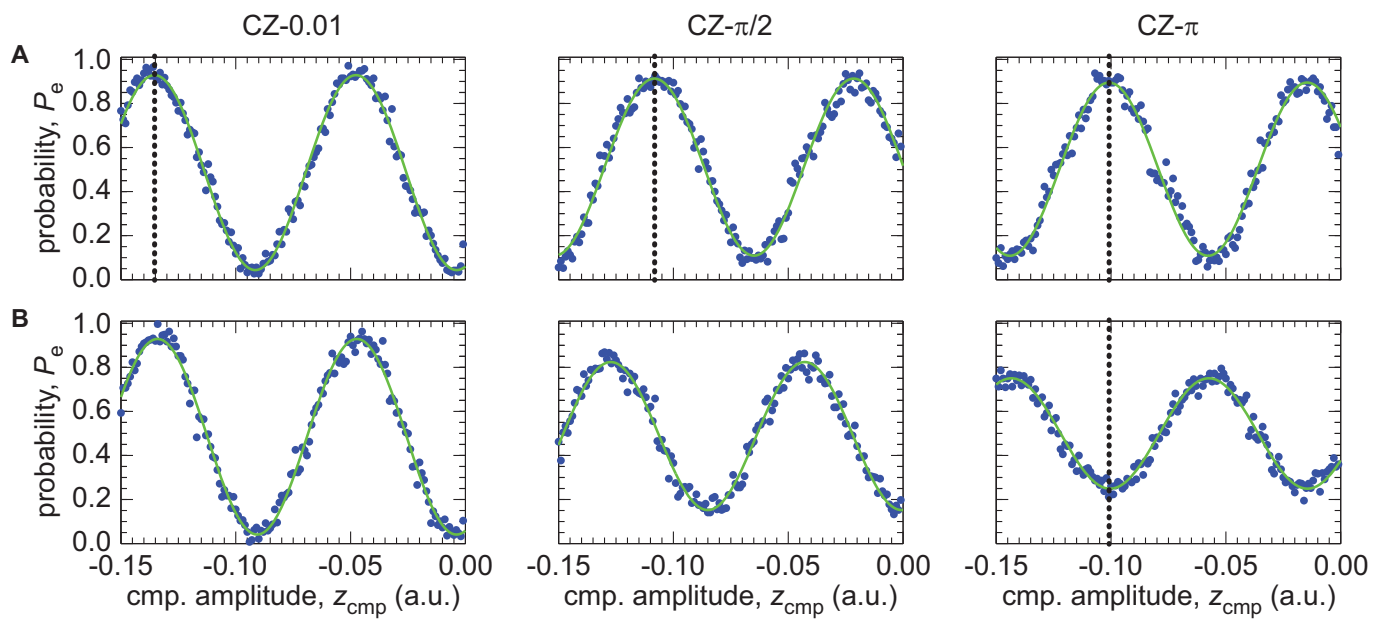

Figure S5: Ramsey experiments for compensating the dynamic phase of $\mathbf{Q}_{1}$ and measuring $\phi$. (A) Probability of measuring $\mathbf{Q}_{1}$ in $|\mathrm{e}\rangle, P_{\mathrm{e}}$, vs. compensation pulse amplitude $z_{\mathrm{cmp}}$ for the pulse sequence of Fig. S4A. The compensation pulse is the z-pulse of step (IVa) in Fig. S4 A. In the CZ- $\phi$ gate experiments we always chose a compensation pulse length $\tau_{\mathrm{cmp}}=7 \mathrm{~ns}$. The blue dots represent measured data, while the solid green lines are least-squares fits to a sine function. From left to right, the panels refer to a CZ-0.01 gate, a CZ- $\pi / 2$ gate, and a CZ- $\pi$ gate, respectively. In each panel, the vertical dotted black line indicates the amplitude $z_{\mathrm{cmp}}$ chosen to compensate the dynamic phase $\phi_{\mathrm{dyn}}$. The $z_{\mathrm{cmp}}$ numerical values expressed in the arbitrary units of our custom electronics are $z_{\mathrm{cmp}} \simeq-0.135$ for the CZ-0.01 gate, $z_{\mathrm{cmp}} \simeq-0.108$ for the CZ- $\pi / 2$ gate, and $z_{\mathrm{cmp}} \simeq-0.101$ for the CZ- $\pi$ gate. Notice that the angle of the CZ-0.01 gate is different than that of the CZ-0.28 gate in the main text. This is because the CZ-0.01 gate shown here is the result of a single set of measurements, whereas the CZ-0.28 gate shown in the main text is the average of a set of 10 independent measurements. (B) Same as in A, but for the pulse sequence of Fig. S4 B. The relative phase between the Ramsey fringes of each panel in A and the corresponding panel in B gives the phase $\phi$ of the CZ- $\phi$ gate. For the three pairs of Ramsey fringes in this example, the relative phases are $\phi=0.01 \mathrm{rad}, \phi=\pi / 2 \mathrm{rad}$, and $\phi=\pi \mathrm{rad}$. The vertical dotted black line in the rightmost panel is positioned at the same value of $z_{\mathrm{cmp}}$ as the corresponding panel in $\mathrm{A}$, but, in this case, it indicates a minimum of the Ramsey fringe because of the $\pi$ shift introduced by the gate.

(Va) A rotation $\hat{R}_{y}^{\pi / 2}$ similar to that in point (IIa) is applied to $\mathrm{Q}_{1}$, bringing the qubit into the final state $\left|\mathrm{Q}_{1}\right\rangle_{(\mathrm{Va})}=$ $-i \sin \left(\phi_{\mathrm{a}} / 2\right)|\mathrm{g}\rangle+\cos \left(\phi_{\mathrm{a}} / 2\right)|\mathrm{e}\rangle$

(VIa) Finally, a measurement pulse is applied to $\mathrm{Q}_{1}$ in order to obtain the probability to find the qubit in $|\mathrm{e}\rangle$, $P_{\mathrm{e}}=\left|\cos \left(\phi_{\mathrm{a}} / 2\right)\right|^{2}=\left(1+\cos \phi_{\mathrm{a}}\right) / 2$. Since $\phi_{\mathrm{a}}$ depends on the compensation pulse amplitude $z_{\mathrm{cmp}}$, the probability $P_{\mathrm{e}}$ is also a function of $z_{\mathrm{cmp}}$. In order to cancel the effect of the dynamic phase $\phi_{\mathrm{dyn}}, z_{\mathrm{cmp}}$ has to be chosen such that $P_{\mathrm{e}}$ reaches a maximum, where the phase $\phi_{\mathrm{a}}=2 K \pi$, with $K \in \mathbb{Z}$. 
In summary, the two qubit rotations $\hat{R}_{y}^{\pi / 2}$ at the beginning and end of the calibration sequence of Fig. S4A realize a generalized Ramsey experiment, which allows us to measure the total phase acquired by $\mathrm{Q}_{1}$ during the z-pulses that bring it outside its reference frame. The experimental data for the calibration sequence of Fig. S4A are shown in Fig. S5.A, where the three Ramsey fringes are obtained for three different values of the detuning $\delta_{\mathrm{Q}_{1} \mathrm{~B}}$, corresponding to a CZ-0.01, CZ- $\pi / 2$, and CZ- $\pi$ gate, respectively. For each Ramsey fringe in Fig. S5 A, the $\mathrm{z}$-pulse amplitude $z_{\mathrm{cmp}}$ chosen to compensate the dynamic phase $\phi_{\mathrm{dyn}}$ is indicated by a vertical dotted black line.

The second calibration sequence for qubit $\mathrm{Q}_{1}$ is shown in Fig. $\mathrm{S} 4 \mathrm{~B}$. The sequence, which is the same as the first calibration sequence with the addition of the pulses applied to qubit $\mathrm{Q}_{2}$, comprises the following steps:

(Ib) Qubit $\mathrm{Q}_{1}$, qubit $\mathrm{Q}_{2}$, and resonator B are initialized in the ground/vacuum state $\left|\mathrm{Q}_{1}\right\rangle_{(\mathrm{Ib})} \otimes\left|\mathrm{Q}_{2}\right\rangle_{(\mathrm{Ib})} \otimes|\mathrm{B}\rangle_{(\mathrm{Ib})}=$ $|\mathrm{g}\rangle \otimes|\mathrm{g}\rangle \otimes|0\rangle$, with both qubits biased at the idle point;

(IIb) A Gaussian microwave pulse with FWHM $\tau_{\text {FWHM }}$ is applied to $\mathrm{Q}_{2}$. The amplitude of the pulse is chosen such that $\Omega_{\mathrm{D}} \tau=\pi$. In this case, the time evolution of $\widehat{\widetilde{H}}_{\mathrm{QD}}$ acting on $\left|\mathrm{Q}_{2}\right\rangle_{(\mathrm{Ib})}$ realizes a full qubit population transfer, $\hat{R}_{y}^{\pi}$, bringing the qubit into the new state $\left|\mathrm{Q}_{2}\right\rangle_{(\mathrm{IIb})}=|\mathrm{e}\rangle$;

(IIIb) The state $\left|\mathrm{Q}_{2}\right\rangle_{\text {(IIb) }}$ is moved from $\mathrm{Q}_{2}$ to $\mathrm{B}$ by means of an iSWAP of length $\tau_{\text {iS }}$. At the end of the iSWAP, resonator $\mathrm{B}$ is in the state $|\mathrm{B}\rangle_{(\mathrm{IIIb})}=|1\rangle$;

(IVb) Qubit $\mathrm{Q}_{1}$ is prepared in the state $\left|\mathrm{Q}_{1}\right\rangle_{(\mathrm{IVb})}=(|\mathrm{g}\rangle+|\mathrm{e}\rangle) / \sqrt{2}$ by means of a rotation $\hat{R}_{y}^{\pi / 2}$;

$(\mathrm{Vb})$ The same z-pulse as in point (IIIa) is applied to $\mathrm{Q}_{1}$. In this case, the $\mathrm{Q}_{1}-\mathrm{B}$ coupled system is in the state $\left.\left|\mathrm{Q}_{1} \mathrm{~B}\right\rangle=(|\mathrm{g}\rangle+|\mathrm{e}\rangle) / \sqrt{2}\right\rangle \otimes|1\rangle$, which represents a bright state of the time evolution of the system, as opposed to the dark state $\left.\left|\mathrm{Q}_{1} \mathrm{~B}\right\rangle=(|\mathrm{g}\rangle+|\mathrm{e}\rangle) / \sqrt{2}\right\rangle \otimes|0\rangle$. Depending on $\tau_{\mathrm{CZ}-\phi}$ and $z_{\mathrm{CZ}-\phi}$, and, thus, on $\delta_{\mathrm{Q}_{1} \mathrm{~B}}$, at the end of the z-pulse the excited state $|\mathrm{e}\rangle$ of $\mathrm{Q}_{1}$ has acquired a phase $\phi$ [cf. main text; when $\phi=\pi$, the $\mathrm{z}$-pulse is a $-\mathrm{SWAP}^{2}[5]$. As in point (IIIa), at the end of such a pulse $\mathrm{Q}_{1}$ has also acquired a dynamic phase $\phi_{\mathrm{dyn}}$, resulting in the state $\left|\mathrm{Q}_{1}\right\rangle_{(\mathrm{Vb})}=\left(|\mathrm{g}\rangle e^{-i \phi_{\mathrm{dyn}}}+|\mathrm{e}\rangle e^{+i \phi_{\mathrm{dyn}}} e^{+i \phi}\right) / \sqrt{2}$;

(VIb) The dynamic phase $\phi_{\text {dyn }}$ acquired by $\mathrm{Q}_{1}$ is compensated by means of a z-pulse, as in point (IVa). At the end of the compensation pulse $\mathrm{Q}_{1}$ is in the state $\left|\mathrm{Q}_{1}\right\rangle_{(\mathrm{VIb})}=\left[|\mathrm{g}\rangle e^{-i \phi_{\mathrm{a}} / 2}+|\mathrm{e}\rangle e^{+i\left(\phi_{\mathrm{a}} / 2+\phi\right)}\right] / \sqrt{2}$;

(VIIb) A rotation $\hat{R}_{y}^{\pi / 2}$ is applied to $\mathrm{Q}_{1}$, bringing the qubit to the final state $\left|\mathrm{Q}_{1}\right\rangle_{(\mathrm{VIIb})}=-i e^{i \phi / 2} \sin \left(\phi_{\mathrm{a}} / 2+\right.$ $\phi / 2)|\mathrm{g}\rangle+e^{i \phi / 2} \cos \left(\phi_{\mathrm{a}} / 2+\phi / 2\right)|\mathrm{e}\rangle$

(VIIIb) Finally, a measurement pulse is applied to $\mathrm{Q}_{1}$ in order to obtain the probability to find the qubit in $|\mathrm{e}\rangle$, $P_{\mathrm{e}}=\left|e^{i \phi / 2} \cos \left(\phi_{\mathrm{a}} / 2+\phi / 2\right)\right|^{2}=\left(1+\cos \phi_{\mathrm{b}}\right) / 2$, where $\phi_{\mathrm{b}} \equiv \phi_{\mathrm{a}}+\phi$.

The phase difference between the probability $P_{\mathrm{e}}$ for the first and second calibration sequence allows us to measure the CZ- $\phi$ gate phase, $\phi_{\mathrm{b}}-\phi_{\mathrm{a}}=\phi$. This is illustrated in Fig. S5. where the phase difference between the Ramsey fringe in each panel of Fig. $\mathrm{S} 5 \mathrm{~A}$ and the corresponding fringe in each panel of Fig. S5B $\mathrm{B}$ gives the phase of a CZ-0.01, CZ- $\pi / 2$, and CZ- $\pi$ gate, respectively.

The second calibration sequence can also be used to cross check the amplitude $z_{\mathrm{cmp}}$ of the compensation pulse chosen to cancel the dynamic phase $\phi_{\mathrm{dyn}}$. For example, when $\phi=\pi$, the Ramsey fringe obtained from the second calibration sequence should reach a minimum for the same value of $z_{\mathrm{cmp}}$ for which it reaches a maximum in the first calibration sequence. This is confirmed by comparing the experimental data shown in the rightmost panel of Fig. S5A and Fig. S5B.

Figure $\mathrm{S6}$ A shows the sequence used to calibrate the pulses applied to qubit $\mathrm{Q}_{2}$ during the $\mathrm{CZ}-\phi$ gate operation. The sequence comprises the following steps:

(Ic) The system is initialized in the state $\left|\mathrm{Q}_{1}\right\rangle_{(\mathrm{Ic})} \otimes\left|\mathrm{Q}_{2}\right\rangle_{(\mathrm{Ic})} \otimes|\mathrm{B}\rangle_{(\mathrm{Ic})}=|\mathrm{g}\rangle \otimes|\mathrm{g}\rangle \otimes|0\rangle$, with both qubits biased at the idle point; 

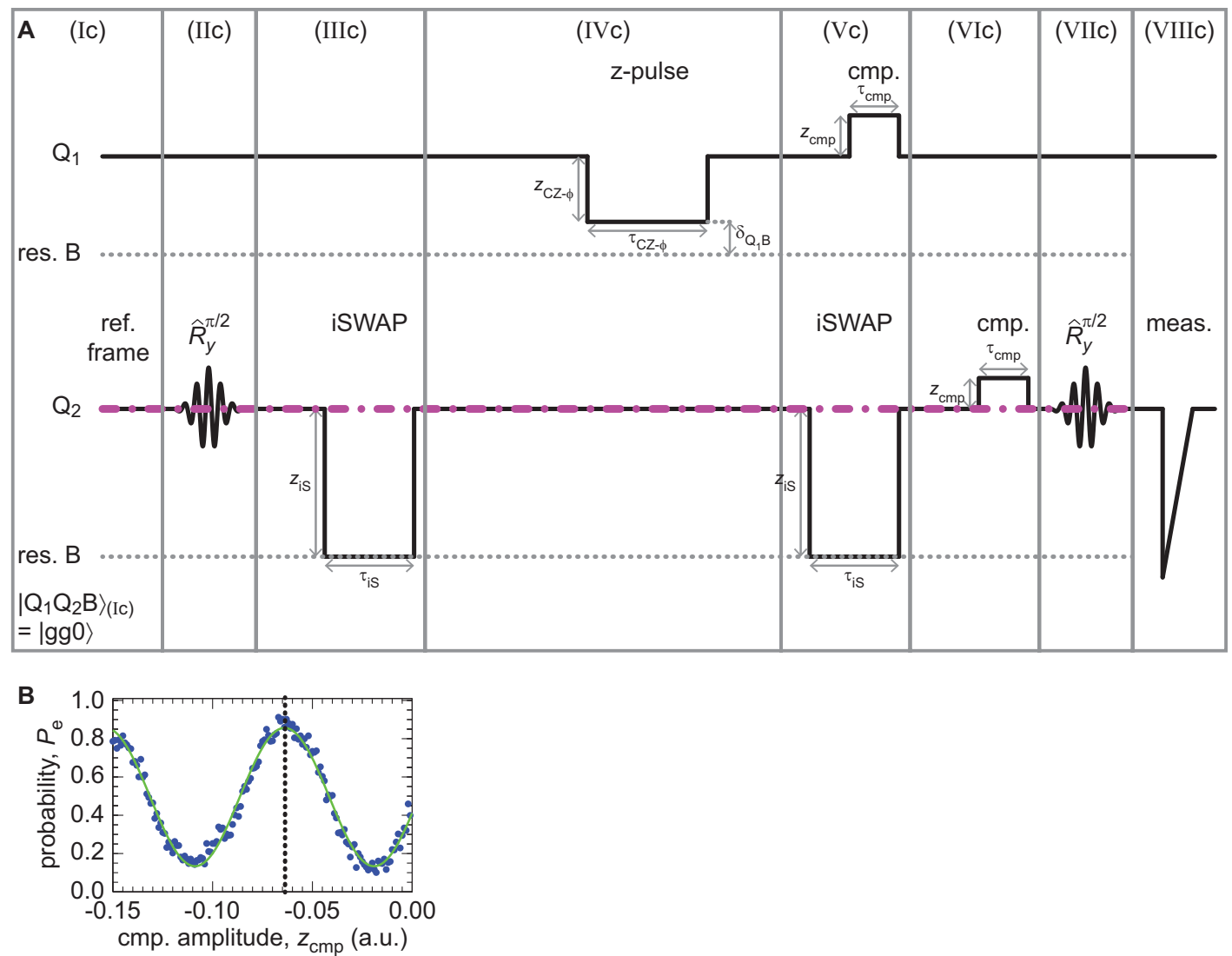

Figure S6: Dynamic phase compensation for qubit $\mathbf{Q}_{2}$. (A) Sequence steps: (Ic), the $\mathrm{Q}_{1}-\mathrm{Q}_{2}-\mathrm{B}$ system is initialized in state $\left|\mathrm{Q}_{1} \mathrm{Q}_{2} \mathrm{~B}\right\rangle_{(\mathrm{Ic})}=|\mathrm{gg} 0\rangle$, with both qubits at the idle point. The reference frame of $\mathrm{Q}_{2}$ is indicated by a dash-dot magenta line. Resonator B is indicated by a dotted grey line; (IIc), rotation $\hat{R}_{y}^{\pi / 2}$ on $\mathrm{Q}_{2}$. The Gaussian pulse has a FWHM $\tau_{\text {FWHM }}=7 \mathrm{~ns}$; (IIIc), iSWAP between $\mathrm{Q}_{2}$ and B with amplitude $z_{\mathrm{iS}}$ and length $\tau_{\mathrm{iS}}=24.97 \mathrm{~ns} ;(\mathrm{IVc})$, z-pulse on $\mathrm{Q}_{1}$ with amplitude $z_{\mathrm{CZ}-\phi}$ and length $\tau_{\mathrm{CZ}-\phi}$; (Vc), iSWAP between B and $\mathrm{Q}_{2}$ with amplitude $z_{\text {is }}$ and length $\tau_{\mathrm{iS}}=24.97 \mathrm{~ns}$. At the same time, compensation pulse on $\mathrm{Q}_{1}$ with amplitude $z_{\mathrm{cmp}}$ and length $\tau_{\mathrm{cmp}}$ set in the sequence of Fig. $\mathrm{S} 43$; (VIc), compensation pulse on $\mathrm{Q}_{2}$ with amplitude $z_{\text {cmp }}$ and length $\tau_{\text {cmp }} ;(\mathrm{VIIc})$, rotation $\hat{R}_{y}^{\pi / 2}$ on $\mathrm{Q}_{2} ;$ (VIIIc), measurement pulse on $\mathrm{Q}_{2}$. (B) Probability of measuring $\mathrm{Q}_{2}$ in $|\mathrm{e}\rangle, P_{\mathrm{e}}$, vs. compensation pulse amplitude $z_{\mathrm{cmp}}$ for the compensation pulse of step (VIc) in A. The blue dots represent measured data, while the solid green line is a least-squares fit to a sine function. The vertical dotted black line indicates the amplitude $z_{\mathrm{cmp}}$ chosen to compensate the dynamic phase acquired by $\mathrm{Q}_{2}$ during the sequence in $\mathrm{A}, z_{\mathrm{cmp}} \simeq-0.064$.

(IIc) A rotation $\hat{R}_{y}^{\pi / 2}$ with FWHM $\tau_{\text {FWHM }}$ is applied to $\mathrm{Q}_{2}$;

(IIIc) The state of $\mathrm{Q}_{2}$ is moved into $\mathrm{B}$ by means of an iSWAP;

(IVc) $\mathrm{Q}_{1}$ is moved on resonance or close to resonance with B through the same z-pulse as point $(\mathrm{Vb})$;

(Vc) The state of $\mathrm{B}$ is moved back to $\mathrm{Q}_{2}$ via an iSWAP. During and between the iSWAPs of point (IIIc) and (Vc), $\mathrm{Q}_{2}$ acquires an unwanted dynamic phase.

At the same time as the iSWAP in point $(\mathrm{Vc})$, the compensation pulse tuned up in the $\mathrm{Q}_{1}$ calibration sequence of Fig. S4 A and B [point (IVa) or (VIb)], is applied to $\mathrm{Q}_{1}$ (this is not strictly necessary due to the independence of the calibration sequences for $\mathrm{Q}_{1}$ and $\mathrm{Q}_{2}$ );

(VIc) A compensation pulse with fixed length $\tau_{\mathrm{cmp}}$ and variable amplitude $z_{\mathrm{cmp}}$ is applied to $\mathrm{Q}_{2}$; 


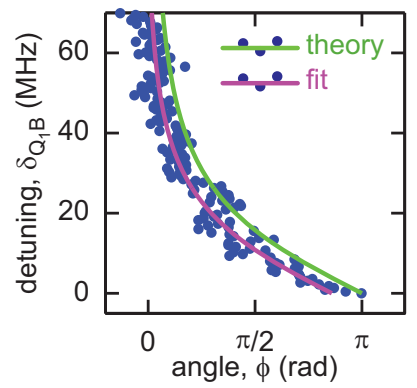

Figure S7: Qubit frequency drift. Phase $\phi$ acquired by $\mathrm{Q}_{1}$ as a function of the detuning $\delta_{\mathrm{Q}_{1} \mathrm{~B}}$. The blue dots indicate the same experimental data as in Fig. 3C of the main text. The solid green line is the theory given by Eq. $\mathrm{S} 24$ and the solid magenta line a fit to the function given by Eq. S25 where $\delta_{\text {drift }}$ is the only free fitting parameter.

(VIIc) A rotation $\hat{R}_{y}^{\pi / 2}$ with FWHM $\tau_{\text {FWHM }}$ is applied to $\mathrm{Q}_{2}$;

(VIIIc) The state $|\mathrm{e}\rangle$ of $\mathrm{Q}_{2}$ is measured, thus obtaining the probability $P_{\mathrm{e}}$ as a function of $z_{\mathrm{cmp}}$ (cf. Fig. $\mathrm{S} 6 \mathrm{~B}$ ). Choosing a maximum of the probability $P_{\mathrm{e}}$ allows us to cancel the effect of the unwanted dynamic phase acquired by $\mathrm{Q}_{2}$ during and between the two iSWAPs.

\section{Systematic errors}

The Ramsey fringes of Fig. S5. A and B, which are used to obtain the phase $\phi$ associated with three particular values of the detuning $\delta_{\mathrm{Q}_{1} \mathrm{~B}}$, can be extended to any arbitrary value of $\delta_{\mathrm{Q}_{1} \mathrm{~B}}$ to obtain all possible values of $\phi$. Figure $3 \mathrm{C}$ in the main text shows, for example, the phase $\phi$ obtained for $\delta_{\mathrm{Q}_{1} \mathrm{~B}} \in[0,70] \mathrm{MHz}$. In the figure, the theoretical expression for the phase $\phi$ given by Eq. $\mathrm{S} 24$ (solid green line) is overlayed to the measured data (blue dots). The coupling $\tilde{g}_{\mathrm{Q}_{1} \mathrm{~B}}$ used to plot the theoretical curve was estimated from the time-domain swaps of Fig. 3B in the main text. A qualitative inspection of the figure shows that theory and measured data are shifted along the vertical axis by a detuning $\delta_{\text {drift }}$, corresponding to an overall phase shift along the horizontal axis.

The origin of the detuning $\delta_{\text {drift }}$, and of the corresponding phase shift, could be attributed to two main causes: (i) - Drift of the transition frequency of qubit $\mathrm{Q}_{1}$ during the experiment; (ii) - drift in the room-temperature electronics. Each pair of Ramsey fringes used to obtain the phase $\phi$ (e.g., the fringe in the leftmost panel of Fig. S5. A and the corresponding fringe in the leftmost panel of Fig. (S5B) was measured within a few minutes. This time can be considered short enough to exclude the electronics drift as a main cause of the detuning between theory and data. We can thus assume the drift in the qubit transition frequency as the main reason for the detuning. This seems a fair assumption since the measurement of the time-domain swaps of Fig. 3B in the main text, which were used to calibrate the z-pulse amplitude $z_{\mathrm{CZ}-\phi}$ and swapping time $\tau_{\mathrm{CZ}-\phi}$ necessary to obtain each phase $\phi$ (cf. main text and the two previous supporting sections on the CZ- $\phi$ gate theory and tuneup), took approximately four hours. Both the swaps and Ramsey fringes were measured starting from large detuning $\delta_{\mathrm{Q}_{1} \mathrm{~B}}=70 \mathrm{MHz}$ to zero detuning, resulting in a time delay between swaps and Ramsey fringes for each value of $\delta_{\mathrm{Q}_{1} \mathrm{~B}}$ of approximately four hours. From independent measurements (not shown), in such a time interval we expect the qubit transition frequency to drift by a few mega hertz.

In order to quantify the detuning $\delta_{\text {drift }}$, we can fit the data of Fig. $3 \mathrm{C}$ in the main text with the function

$$
\phi \equiv \pi-\pi \frac{\delta_{\mathrm{Q}_{1} \mathrm{~B}}}{\sqrt{\delta_{\mathrm{Q}_{1} \mathrm{~B}}^{2}+\tilde{g}_{\mathrm{Q}_{1} \mathrm{~B}}^{2}}}+\pi \frac{\delta_{\mathrm{drift}}}{\sqrt{\delta_{\mathrm{Q}_{1} \mathrm{~B}}^{2}+\tilde{g}_{\mathrm{Q}_{1} \mathrm{~B}}^{2}}} .
$$

The function of Eq. $\mathrm{S} 25$ represents the best fit we found for the data of Fig. 3C in the main text. Figure S7] shows the same data as Fig. 3C of the main text (blue dots), together with the theory of Eq. S24 (solid green line), and 
the fit of Eq. S25 (solid magenta line). The detuning obtained from the fit is $\left|\delta_{\text {drift }}\right| \simeq 4 \mathrm{MHz}$, which is consistent with our expectation for a qubit frequency drift in approximately four hours.

We note that the qubit frequency drift as well as the data scatter in Fig. 3C of the main text (or, equivalently, of Fig. S7 are peculiar to that measurement, where we intended to show all phases $\phi \in(0, \pi]$ in a single, long scan. When it will be required to use a specific phase $\phi$ to perform a quantum Fourier transform during an algorithm, we will first theoretically estimate the parameters $z_{\mathrm{CZ}-\phi}$ and $\tau_{\mathrm{CZ}-\phi}$ and, then, search for the phase $\phi$ in the close vicinity of these parameters. This will allow us to measure only a small portion of the swaps of Fig. 3B in the main text and a few corresponding Ramsey fringes, which can be realized in a much shorter time than the long scan of Fig. $3 \mathrm{C}$ in the main text, thus significantly reducing the incidence of systematic errors.

\section{XOR gate and $M$ gate tuneup}

In this section, we describe the experimental pulse sequences required to tune up the XOR gate and $\mathrm{M}$ gate shown in the main text. We also explain in detail the complete experimental sequence used to obtain one nontrivial entry of the truth table associated with the M gate. Finally, we outline the mathematical procedure at the basis of quantum phase tomography. 

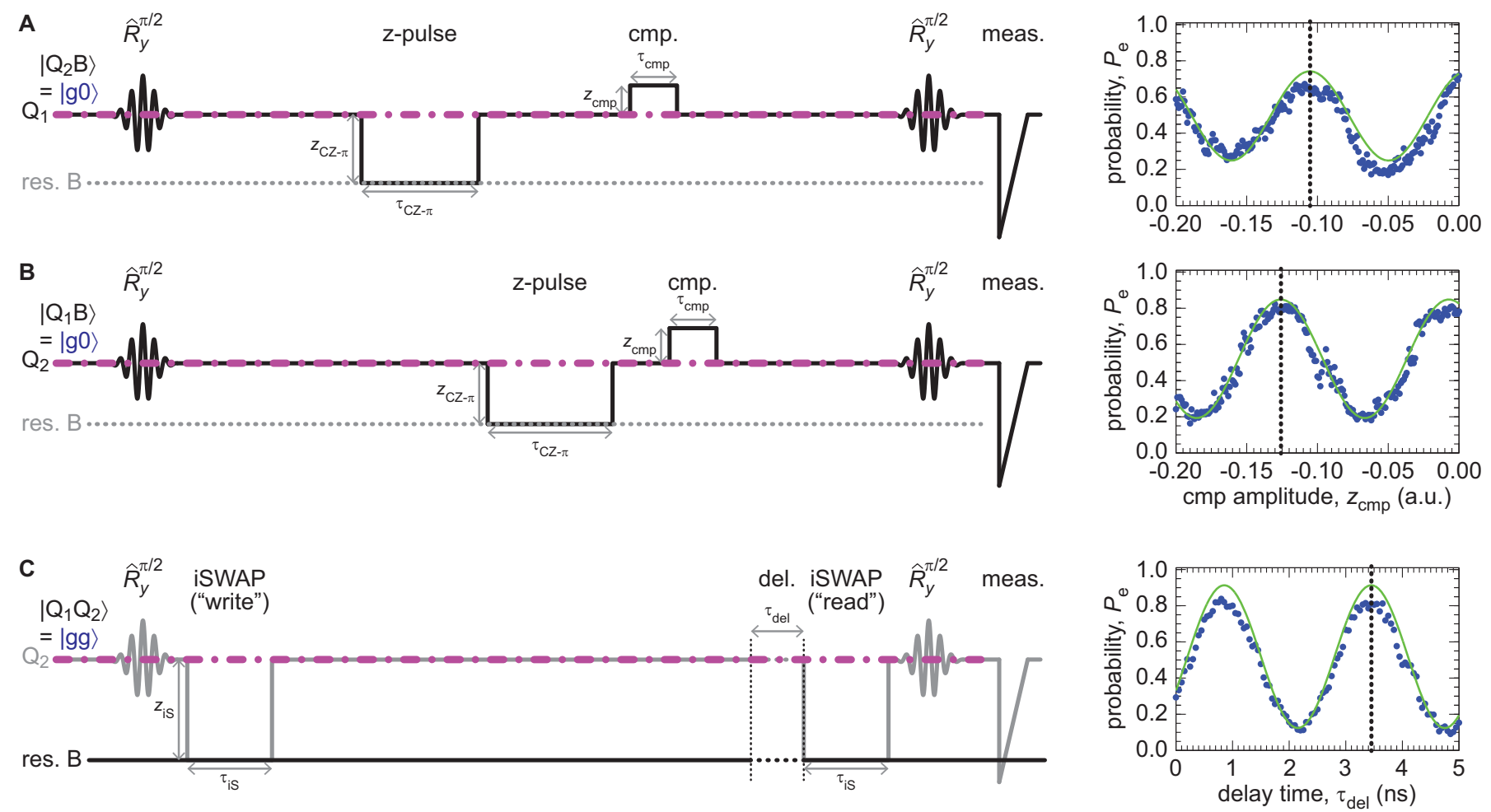

Figure S8: XOR gate tuneup. (A) (Left) Tuneup sequence (1-XOR). Qubit $\mathrm{Q}_{1}$ is initialized in $|\mathrm{g}\rangle$ at the idle point. Qubit $\mathrm{Q}_{2}$ and resonator $\mathrm{B}$ remain in state $\left|\mathrm{Q}_{2} \mathrm{~B}\right\rangle=|\mathrm{g} 0\rangle$ during the whole sequence. The reference frame of $\mathrm{Q}_{1}$ is indicated by a dash-dot magenta line. Resonator $\mathrm{B}$ is indicated by a dotted grey line. (Right) Ramsey fringe corresponding to the sequence on the left showing the probability of measuring $\mathrm{Q}_{1}$ in $|\mathrm{e}\rangle, P_{\mathrm{e}}$, plotted vs. $z_{\mathrm{cmp}}$. The blue dots represent measured data, and the solid green line a least-squares fit to a sine function. The vertical dotted black line indicates the amplitude $z_{\mathrm{cmp}}$, obtained from the fit, chosen to compensate the dynamic phase acquired by $\mathrm{Q}_{1}$ during the first z-pulse in the sequence. (B) Tuneup sequence (2-XOR). As in A, but for $\mathrm{Q}_{2}$. (C) (Left) Tuneup sequence (3-XOR). The solid grey line indicates the ancilla qubit $\mathrm{Q}_{2}$, and the solid black line resonator $\mathrm{B}$. The reference frame is set by $\mathrm{Q}_{2}$ at the idle point, as indicated by the dash-dot magenta line. (Right) Ramsey fringe corresponding to the sequence on the left, where the probability of measuring $\mathrm{Q}_{2}$ in $|\mathrm{e}\rangle, P_{\mathrm{e}}$, is plotted vs. $\tau_{\text {del }}$. The blue dots represent measured data, and the solid green line a least-squares fit to a sine function. The vertical dotted black line indicates the time $\tau_{\mathrm{del}}$, obtained from the fit, chosen to calibrate away the dynamic phase acquired by the state in B during and between the "write" and "read" iSWAP. 


\section{XOR gate tuneup}

The quantum logic circuit of the XOR gate considered here is sketched in Fig. 4A of the main text. Figure S8, A to $\mathrm{C}$, shows the three sequences and the corresponding Ramsey fringes required to tune up the XOR gate. The concept behind each sequence is similar to the compensation of a dynamic phase acquired during a CZ- $\phi$ gate, which has been elucidated in the section "The quantum Fourier transform" of these Methods. The third sequence needs special attention as it is applied to a resonator rather than a qubit state.

(1-XOR) The first sequence, which is displayed in Fig. $\mathrm{S} 8 \mathrm{~A}$ (Left), acts on control qubit $\mathrm{Q}_{1}$. During the entire sequence, the control qubit $\mathrm{Q}_{2}$ and the target bus resonator $\mathrm{B}$ remain in the state $\left|\mathrm{Q}_{2} \mathrm{~B}\right\rangle=|\mathrm{g} 0\rangle$, and all pulses acting on $Q_{2}$ are turned off. Qubit $Q_{1}$ is initialized in the ground state $\left|Q_{1}\right\rangle=|\mathrm{g}\rangle$ at the idle point.

As for the case of the CZ- $\phi$ gate, the sequence consists of a Ramsey-type experiment used to determine and calibrate away the total dynamic phase acquired by $\mathrm{Q}_{1}$ during the XOR gate. Hence, the first step of the sequence is an $\hat{R}_{y}^{\pi / 2}$ unitary rotation on $\mathrm{Q}_{1}$, which brings the qubit to the equator of the Bloch sphere.

Then, a z-pulse with amplitude $z_{\mathrm{CZ}-\pi}$ and time $\tau_{\mathrm{CZ}-\pi} \simeq 39.08 \mathrm{~ns}$, brings the $|\mathrm{e}\rangle \leftrightarrow|\mathrm{f}\rangle$ transition of $\mathrm{Q}_{1}$ into resonance with $\mathrm{B}$. The time $\tau_{\mathrm{CZ}-\pi}$ is inversely proportional to the coupling strength between states $\left|\mathrm{Q}_{1} \mathrm{~B}\right\rangle=|\mathrm{e} 1\rangle$ and $|\mathrm{f} 0\rangle$. However, we note that in the sequence (1-XOR), B is always in the vacuum state $|0\rangle$ and, thus, no dynamics takes place between $\mathrm{Q}_{1}$ and $\mathrm{B}$ during the z-pulse. The only effect of the z-pulse is to detune $\mathrm{Q}_{1}$ outside its reference frame, which causes the qubit to acquire an unwanted dynamic phase $\phi_{\text {dyn }}$.

In order to compensate $\phi_{\text {dyn }}$, a second z-pulse must be applied to $\mathrm{Q}_{1}$. Such a pulse is characterized by a fixed time-length $\tau_{\mathrm{cmp}}=5 \mathrm{~ns}$ and a variable amplitude $z_{\mathrm{cmp}}$. We note that all compensation pulses in the XOR- and M-gate tuneup sequences have the same time length of $5 \mathrm{~ns}$. By continuously varying the amplitude $z_{\text {cmp }}$, the Ramsey fringe shown in Fig. $\mathrm{S} 8 \mathrm{~A}$ (Right) is obtained. The dynamic phase $\phi_{\mathrm{dyn}}$ is totally compensated when the probability $P_{\mathrm{e}}$ reaches a maximum. In the figure, the vertical dotted black line indicates the compensation pulse amplitude chosen for this purpose, $z_{\mathrm{cmp}} \simeq-0.106$;

(2-XOR) The second tuneup sequence is displayed in Fig. $[\mathrm{S} 8 \mathrm{~B}$ (Left). The sequence is analogous to sequence (1$\mathrm{XOR}$ ), but acting on control qubit $\mathrm{Q}_{2}$ instead of $\mathrm{Q}_{1}$. In this case, $z_{\mathrm{cmp}} \simeq-0.127$ [cf. Fig. S88 (Right)];

(3-XOR) The third and last tuneup sequence of the XOR gate, which is shown in Fig. S8C (Left), acts on target resonator B. Sequence (3-XOR) represents a departure from the analogy between the tuneup of the XOR gate and the $\mathrm{CZ}-\phi$ gate, where only two compensation pulses were needed for the gate operation.

As already explained in the main text, resonator B plays the role of the third qubit in our implementation of three-qubit phase gates. In order to use resonator $\mathrm{B}$ as an effective qubit, its state must be prepared and measured using either qubit $\mathrm{Q}_{1}$ or $\mathrm{Q}_{2}$ as an ancilla qubit. In our experiments, we have chosen $\mathrm{Q}_{2}$ to perform this function because of slightly better coherence times and measurement fidelities compared to $Q_{1}$. It is important to note that $\mathrm{Q}_{2}$ is actively used during the XOR gate. Consequently, the state of $\mathrm{B}$ can only be controlled before and/or after the gate operation. This issue does not constitute an experimental limitation since B represents the target of the gate and, thus, its state will not be controlled during the gate. However, once a state has been loaded in target $\mathrm{B}$, it has to remain stored for a significantly longer time than any state stored in the control qubits $\mathrm{Q}_{1}$ and $\mathrm{Q}_{2}$. This is not an experimental limitation either, as the much longer coherence times of B compared to $\mathrm{Q}_{1}$ and $\mathrm{Q}_{2}$ (cf. caption of Fig. 1B in the main text for numerical values) largely reduce the effect of a longer storing time. This experiment further proves the importance of the quantum von Neumann architecture, where the ability to store states in a memory makes possible to realize longer quantum computations.

As for the control qubits $\mathrm{Q}_{1}$ and $\mathrm{Q}_{2}$, also the state loaded in the target resonator $\mathrm{B}$ acquires a dynamic phase due to the detuning between the transition frequency of $B$ and the reference clock rate of $Q_{2}$. Note that only the frequency detuning with respect to $Q_{2}$ contributes to the dynamic phase of the state in $B$ because $Q_{2}$ is the ancilla qubit chosen to manipulate and measure $\mathrm{B}$. 
Following the pulses in Fig. $\overline{S 8 C}$ (Left), the Ramsey experiment necessary to compensate the dynamic phase acquired by $B$ is indirectly preformed through $\mathrm{Q}_{2}{ }^{15 / 16}$. Resonator $\mathrm{B}$ is initialized in the vacuum state $|B\rangle=|0\rangle$, and qubit $\mathrm{Q}_{1}$ and $\mathrm{Q}_{2}$ in the state $\left|\mathrm{Q}_{1} \mathrm{Q}_{2}\right\rangle=|\mathrm{gg}\rangle$, with both qubits at the idle point. While $\mathrm{Q}_{1}$ remains in $|\mathrm{g}\rangle$ during the whole sequence, $\mathrm{Q}_{2}$ is rotated into a linear superposition $|\mathrm{g}\rangle+|\mathrm{e}\rangle$ by means of an $\hat{R}_{y}^{\pi / 2}$ rotation. Afterwards, an iSWAP with amplitude $z_{\text {iS }}$ and time $\tau_{\text {iS }} \simeq 25.72 \mathrm{~ns}$ is used to write the state from $\mathrm{Q}_{2}$ into $\mathrm{B}$, which is thus prepared in the state $|0\rangle+|1\rangle$. This state remains loaded in $\mathrm{B}$ until it is read out by $\mathrm{Q}_{2}$ via a second iSWAP before the end of the sequence. Between the two iSWAPs, the control qubits $\mathrm{Q}_{1}$ and $\mathrm{Q}_{2}$ remain in the state $\left|\mathrm{Q}_{1} \mathrm{Q}_{2}\right\rangle=|\mathrm{gg}\rangle$, and all pulses acting on both $\mathrm{Q}_{1}$ and $\mathrm{Q}_{2}$ are turned off. Due to the excursion outside $\mathrm{Q}_{2}$ 's reference frame, the state $|0\rangle+|1\rangle$ in $\mathrm{B}$ acquires a dynamic phase, which grows until the end of the readout iSWAP. Since the resonance frequency of B cannot be tuned, in order to calibrate away the effect of such a dynamic phase we delay the starting time of the readout iSWAP by a variable time $\tau_{\text {del }}$.

Finally, after the readout iSWAP, a second $\hat{R}_{y}^{\pi / 2}$ rotation followed by a measurement pulse on $\mathrm{Q}_{2}$ completes the Ramsey experiment on B. The corresponding Ramsey fringe measured as a function of $\tau_{\text {del }}$ is plotted in Fig. S8C (Right). Similar to sequence (1-XOR) and (2-XOR), choosing $\tau_{\text {del }}$ such that the Ramsey fringe reaches one maximum allows us to fully compensate the dynamic phase acquired by the state in $\mathrm{B}$. The vertical dashed black line in the figure indicates the delay time chosen in the experiment, $\tau_{\text {del }} \simeq 3.46 \mathrm{~ns}$. As a check, from the fit we also obtained a Ramsey fringe frequency of $\simeq 385.0 \mathrm{MHz}$, which agrees well with the $\mathrm{Q}_{2}$-B detuning $\simeq 369.8 \mathrm{MHz}$.

\section{M gate tuneup}

The quantum logic circuit of the M gate considered here is sketched in Fig. 4D of the main text. Figure S9, A to $\mathrm{E}$, shows the five sequences and the corresponding Ramsey fringes required to tune up the $\mathrm{M}$ gate. The tuneup concept is similar to that used for the XOR gate, but with a few important differences due to the $1 / 2 \mathrm{CZ}-\pi$ gates.

(1-M) The first tuneup sequence for the $\mathrm{M}$ gate, which is displayed in Fig. S9A (Left), acts on control qubit $\mathrm{Q}_{1}$. The only difference between this sequence and sequence (1-XOR) is that the single z-pulse with amplitude $z_{\mathrm{CZ}-\pi}$ and length $\tau_{\mathrm{CZ}-\pi}$ is now split into two z-pulses, z-pulse $\alpha$ and z-pulse $\beta$, with amplitude $z_{1 / 2 \mathrm{CZ}-\pi}=z_{\mathrm{CZ}-\pi}$

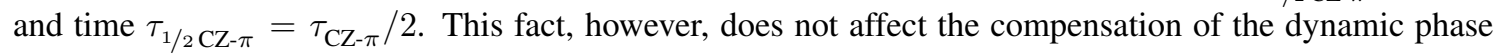
acquired by $\mathrm{Q}_{1}$, as the total excursion of $\mathrm{Q}_{1}$ outside its reference frame remains unchanged. It is worth reminding that the z-pulse $\alpha$ and z-pulse $\beta$ bring the qubit $|\mathrm{e}\rangle \leftrightarrow|\mathrm{f}\rangle$ transition on resonance with bus resonator B. As a consequence, in sequence (1-M) the resonator remains in the vacuum state $|0\rangle$.

From the Ramsey fringe in Fig. [S9A (Right) we obtain one possible value of the compensation pulse amplitude that maximizes the probability $P_{\mathrm{e}}$ of $\mathrm{Q}_{1}, z_{\mathrm{cmp}} \simeq-0.119$

(2-M) The second tuneup sequence, which is displayed in Fig. $\mathrm{S9B}$ (Left), acts on control qubit $\mathrm{Q}_{2}$. The sequence is the same as sequence (2-XOR) for the XOR gate. As in sequence (1-M), the z-pulse brings the qubit $|\mathrm{e}\rangle \leftrightarrow|\mathrm{f}\rangle$ transition on resonance with bus resonator $\mathrm{B}$.

From the Ramsey fringe in Fig. $\mathrm{S9}$ B (Right) we obtain one possible value of the compensation pulse amplitude that maximizes the probability $P_{\mathrm{e}}$ of $\mathrm{Q}_{2}, z_{\mathrm{cmp}} \simeq-0.077$;

(3-M) The third tuneup sequence, which is displayed in Fig. $S 9 \mathrm{C}$ (Left), acts again on control qubit $\mathrm{Q}_{1}$, with control qubit $\mathrm{Q}_{2}$ and target resonator $\mathrm{B}$ either in state $\left|\mathrm{Q}_{2} \mathrm{~B}\right\rangle=|\mathrm{g} 0\rangle$ or $\left|\mathrm{Q}_{2} \mathrm{~B}\right\rangle=|\mathrm{g} 1\rangle$. In addition, all pulses acting on $\mathrm{Q}_{2}$ are turned off. Qubit $\mathrm{Q}_{1}$ is initialized in the ground state $\left|\mathrm{Q}_{1}\right\rangle=|\mathrm{g}\rangle$ at the idle point.

In order to understand the dynamics of the interaction between $\mathrm{Q}_{1}$ and $\mathrm{B}$, we refer to the energy diagram of Fig. $\mathrm{S} 3 \mathrm{~A}$. If $|\mathrm{B}\rangle=|0\rangle$, after the first $\hat{R}_{y}^{\pi / 2}$ rotation on $\mathrm{Q}_{1}$, the $\mathrm{Q}_{1}-\mathrm{B}$ coupled system is in state $\left|\mathrm{Q}_{1}\right\rangle \otimes|\mathrm{B}\rangle=$ $(|\mathrm{g}\rangle+|\mathrm{e}\rangle) \otimes|0\rangle$. In this case, during the z-pulse $\alpha$ and $\mathrm{z}$-pulse $\beta$ no dynamics takes place. As a consequence, in the time interval $\tau_{\text {sh }}$ between the two z-pulses, $\mathrm{Q}_{1}$ remains biased at the idle point in state $\left|\mathrm{Q}_{1}\right\rangle=|\mathrm{g}\rangle+|\mathrm{e}\rangle$, 


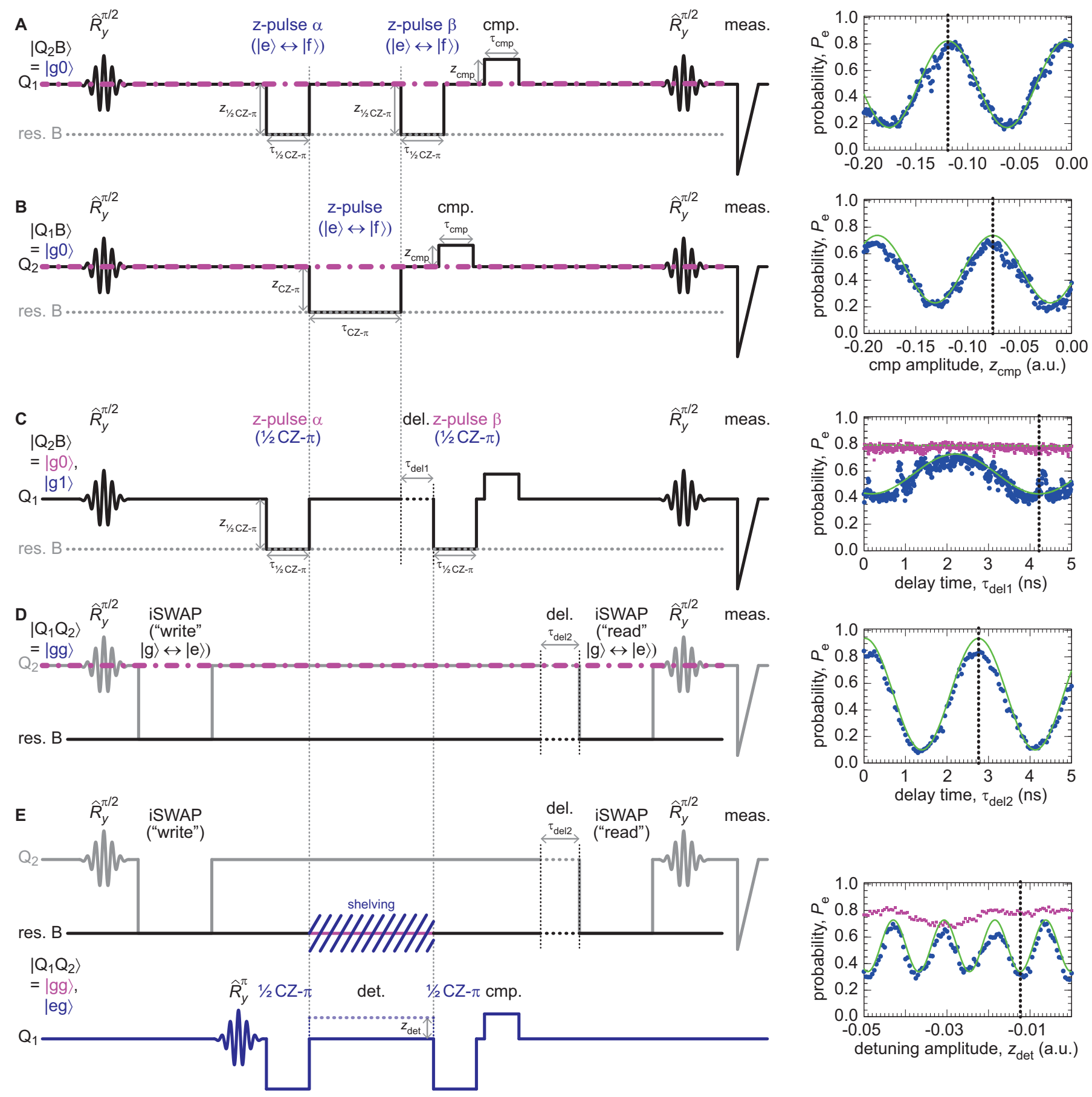

Figure S9: M gate tuneup. (A) Sequence (1-M) for calibrating the $1 / 2 \mathrm{CZ}-\pi$ gates. (B) Same sequence as in Fig. $\mathrm{S} 8 \mathrm{~B}$. (C) (Left) Sequence (3-M) for compensating the dynamic phase acquired by $\mathrm{Q}_{1}$ during the shelving. $\tau_{\text {del1 }}$ : Time delay between the $\mathrm{z}$-pulse $\alpha$ and z-pulse $\beta$ (i.e., between the two $1 / 2$ CZ- $\pi$ gates). (Right) Probability $P_{\mathrm{e}}$ vs. $\tau_{\text {del } 1}$ for $\left|\mathrm{Q}_{2} \mathrm{~B}\right\rangle=|\mathrm{g} 0\rangle$ (magenta squares) or $\left|\mathrm{Q}_{2} \mathrm{~B}\right\rangle=|\mathrm{g} 1\rangle$ (blue dots). Dashed and solid green lines: Least-squares fit to data. (D) Same sequence as in Fig. S8C. (E) As in C, but for bus resonator B. In this case, a detuning $z_{\text {det }}$ is applied between the two $1 / 2$ CZ- $\pi$ gates. No shelving is indicated by a solid magenta line, shelving by a hashed blue. 
without acquiring any dynamic phase. The only dynamic phase acquired by $\mathrm{Q}_{1}$ is that developed during the z-pulse $\alpha$ and z-pulse $\beta$, which has already been compensated in sequence (1-M) (cf. Fig. S9A). The compensation pulse for such a dynamic phase remains turned on during sequence (3-M).

If instead $|\mathrm{B}\rangle=|1\rangle$, after the first $\hat{R}_{y}^{\pi / 2}$ rotation on $\mathrm{Q}_{1}$, the $\mathrm{Q}_{1}-\mathrm{B}$ coupled system is in state $\left|\mathrm{Q}_{1}\right\rangle \otimes|\mathrm{B}\rangle=$ $(|\mathrm{g}\rangle+|\mathrm{e}\rangle) \otimes|1\rangle$. In this case, the reference clock rate is given by $f_{\mathrm{Q}_{1}}+f_{\mathrm{B}}$. After the z-pulse $\alpha$, the state $\left|\mathrm{Q}_{1} \mathrm{~B}\right\rangle=|\mathrm{e} 1\rangle$ gets shelved into the state $\left|\mathrm{Q}_{1} \mathrm{~B}\right\rangle=|\mathrm{f} 0\rangle$ for a time $\tau_{\text {sh }}$, at the end of which the z-pulse $\beta$ is applied. We remind that the $|\mathrm{g}\rangle \leftrightarrow|\mathrm{f}\rangle$ frequency is $\left(2 f_{\mathrm{Q}_{1}}-\delta_{\mathrm{nl}}\right)$, where $\delta_{\mathrm{nl}}$ is the qubit nonlinearity defined as the frequency difference between the $|\mathrm{e}\rangle \leftrightarrow|\mathrm{f}\rangle$ and the $|\mathrm{g}\rangle \leftrightarrow|\mathrm{e}\rangle$ qubit transitions. In this experiment, $f_{\mathrm{Q}_{1}} \simeq 7.2161 \mathrm{GHz}, \delta_{\mathrm{nl}} \simeq 140.6 \mathrm{MHz}$, and $f_{\mathrm{B}} \simeq 6.8150 \mathrm{GHz}$. During the time $\tau_{\mathrm{sh}}$, the coupled system is in state $\left|\mathrm{Q}_{1} \mathrm{~B}\right\rangle=(|\mathrm{g} 1\rangle+|\mathrm{f} 0\rangle)$ and $\mathrm{Q}_{1}$ acquires a dynamic phase $\phi_{\mathrm{sh}}=\left(f_{\mathrm{Q}_{1}}-\delta_{\mathrm{nl}}-f_{\mathrm{B}}\right) \tau_{\mathrm{sh}}$. This dynamic phase is independent from the dynamic phase acquired during the z-pulse $\alpha$ and z-pulse $\beta$.

In order to compensate the phase $\phi_{\text {sh }}$, we delay the starting point of the z-pulse $\beta$ by a time $\tau_{\text {del1 }}$. By continuously varying $\tau_{\text {del1 }}$, the two Ramsey fringes plotted in Fig. S9C (Right) are obtained. The magenta squares correspond to the case $\left|\mathrm{Q}_{2} \mathrm{~B}\right\rangle=|\mathrm{g} 0\rangle$. As expected, in this case nothing happens as the dynamic phases acquired during the z-pulse $\alpha$ and z-pulse $\beta$ were already corrected by the compensation pulse tuned up in sequence (1-M). The Ramsey fringe thus remains at the maximum probability chosen in that sequence. This fringe indicates that no resonator state has been shelved to the qutrit state $\left|Q_{1}\right\rangle=|f\rangle$. The blue dots, instead, correspond to the case $\left|Q_{2} B\right\rangle=|\mathrm{g} 1\rangle$. In this case, the sinusoidal dependence of the fringe clearly shows an excursion outside the $\mathrm{Q}_{1}$-B reference frame, which causes the dynamic phase $\phi_{\mathrm{sh}}$ to be acquired by the state in $\mathrm{Q}_{1}$. This phase is totally compensated when the probability $P_{\mathrm{e}}$ of $\mathrm{Q}_{1}$ reaches a minimum. The reason why a minimum has to be chosen is because the Ramsey fringe is obtained with one excitation in the system, instead of no excitation as in sequences (1-M) and (2-M). This is analogous to the CZ- $\phi$ gate tuneup sequence displayed in Fig. S4B. This can also be understood from the phase-gate cube of Fig. SS11E (or the second row in Table S3), as the Ramsey fringe in sequence (3-M) measures the phase difference between vertex (5) and vertex (1) of the cube, which is $\pi \mathrm{rad}$ instead of $0 \mathrm{rad}$. The vertical dotted black line in Fig. S9P (Right) indicates the delay time chosen in the experiment, $\tau_{\text {del1 }} \simeq 4.2 \mathrm{~ns}$. As a check, a leastsquares fit to the data (solid green line) allows us to extract the frequency of the Ramsey fringe, which is $\simeq 248.2 \mathrm{MHz}$. This number is close to the expected value $\left(f_{\mathrm{Q}_{1}}-\delta_{\mathrm{nl}}-f_{\mathrm{B}}\right) \simeq 260.5 \mathrm{MHz}$ (note that the data consists of one Ramsey oscillation period only. Hence, the $\simeq 10 \mathrm{MHz}$ difference between the theoretically expected value and that obtained from the fit is within the fit confidence interval);

(4-M) The fourth tuneup sequence, which is displayed in Fig. S9P (Left), acts on target resonator B. The sequence is the same as sequence (3-XOR) for the XOR gate. In this sequence, the two iSWAPs bring the qubit $|\mathrm{g}\rangle \leftrightarrow|\mathrm{e}\rangle$ transition on resonance with bus resonator $\mathrm{B}$.

From the Ramsey fringe in Fig. S9D (Right) we obtain one possible value of the delay time that maximizes the probability $P_{\mathrm{e}}$ of $\mathrm{Q}_{2}, \tau_{\text {del } 2} \simeq 2.77 \mathrm{~ns}$ (vertical dotted black line). Since the XOR gate and $\mathrm{M}$ gate experiments were performed several hours apart, the delay time for the $\mathrm{M}$ gate differs slightly from that for the XOR gate because of drifts in the qubit transition frequency (cf. section on "Systematic errors" in these Supporting Online Material). In addition, from a least-squares fit to the data (solid green line) we extracted a Ramsey fringe frequency $\simeq 365.8 \mathrm{MHz}$, which agrees well with the detuning between the reference clock rate of $\mathrm{Q}_{2}$ and the transition frequency of $\mathrm{B}, \simeq 369.8 \mathrm{MHz}$;

(5-M) The fifth tuneup sequence, which is displayed in Fig. S9E (Left), acts again on target resonator B.

Sequence (3-M) served to compensate the dynamic phase acquired by $\mathrm{Q}_{1}$ during the shelving dynamics. Because B takes also part in the shelving, its state acquires a similar dynamic phase that must be compensated. Sequence (5-M) is analogous to sequence (3-M), with two differences. First, the Ramsey experiment is now performed on $B$ via $Q_{2}$ [as for sequence (4-M)]. Second, instead of adding a time delay, qubit $Q_{1}$ is detuned in the $\mathrm{z}$ direction by a quantity $z_{\text {det }}$ for the entire interval between the $\mathrm{z}$-pulse $\alpha$ and $\mathrm{z}$-pulse $\beta$. In fact, we are not allowed to use two times the same degree of freedom, i.e., the delay time $\tau_{\text {del } 1}$ of 


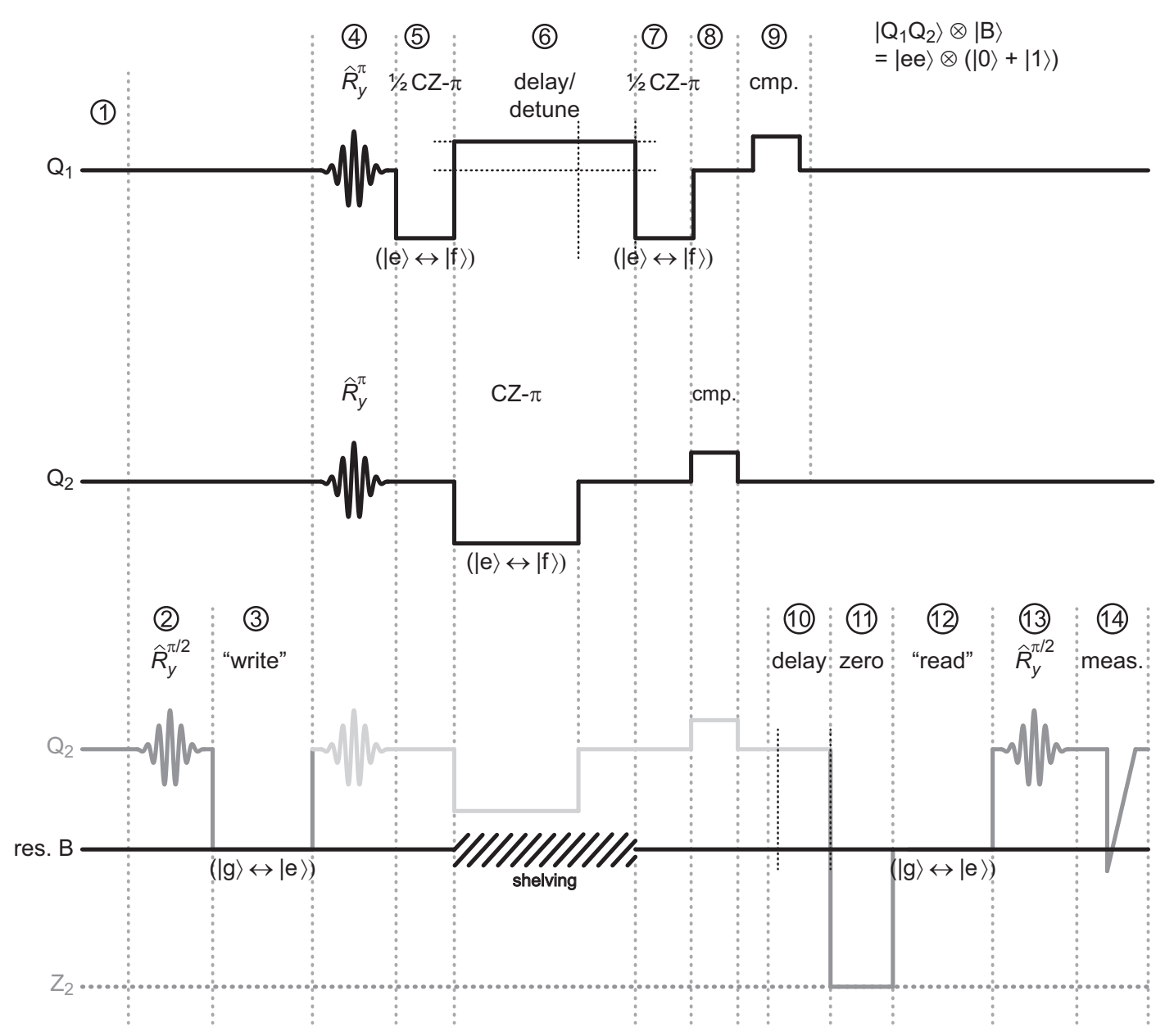

Figure S10: M gate pulse sequence. Pulse sequence used to measure the Ramsey fringe in Fig. 4E of the main text (magenta dots). The vertical dotted grey lines separate the sequence in 14 time frames. Each frame is described in the text.

sequence (3-M), for the compensation of two independent dynamic phases. By continuously varying $z_{\mathrm{det}}$, the two Ramsey fringes plotted in Fig. S9E (Right) are obtained. The interpretation of the fringes is the same as for sequence (3-M). The detuning value chosen in the experiment to compensate the dynamic phase acquired by the state in $\mathrm{B}$ during the shelving is $z_{\mathrm{det}} \simeq-0.012$ (vertical dotted black line);

(6-M) The sixth and last tuneup sequence for the $\mathrm{M}$ gate, which is not shown in Fig. S9, consists in repeating sequence (1-M). The compensation pulse for $\mathrm{Q}_{1}$ needs to be recalibrated due to the detuning $z_{\text {det }}$ set in sequence (5-M). The final value of the compensation pulse amplitude for $\mathrm{Q}_{1}$ chosen in the experiment is $z_{\mathrm{cmp}} \simeq-0.125$.

\section{M gate pulse sequence}

Figure $\mathrm{S} 10$ shows the complete pulse sequence utilized to measure the entry of the $\mathrm{M}$ gate truth table associated with state $\left|\mathrm{Q}_{1} \mathrm{Q}_{2}\right\rangle \otimes|\mathrm{B}\rangle=\mid$ ee $\rangle \otimes(|0\rangle+|1\rangle)$. Step (1): Both control qubits $\mathrm{Q}_{1}$ and $\mathrm{Q}_{2}$ and target resonator $\mathrm{B}$ are initialized in the ground state, $\left|\mathrm{Q}_{1} \mathrm{Q}_{2} \mathrm{~B}\right\rangle=|\operatorname{gg} 0\rangle$. The qubits are biased at the idle point. Step (2): $\mathrm{Q}_{2}$ is prepared in state $|\mathrm{g}\rangle+|\mathrm{e}\rangle$ by means of an $\hat{R}_{y}^{\pi / 2}$ rotation. Step (3): The state in qubit $\mathrm{Q}_{2}$ is written into $\mathrm{B}$ via an iSWAP. 
Until this step, qubit $\mathrm{Q}_{2}$ serves as ancilla qubit to load resonator B. The iSWAP effectively zeros $\mathrm{Q}_{2}$, which can be now used as a control qubit in the $\mathrm{M}$ gate. Step (4): Both control qubits $\mathrm{Q}_{1}$ and $\mathrm{Q}_{2}$ are loaded in state $|\mathrm{e}\rangle$ by means of an $\hat{R}_{y}^{\pi}$ rotation. Step (5): First ${ }^{1 / 2} \mathrm{CZ}-\pi$ gate between $\mathrm{Q}_{1}$ and B. Step (6): CZ- $\pi$ gate between $\mathrm{Q}_{2}$ and $B$. In the same time frame, the delay and detune necessary to compensate the dynamic phase on $\mathrm{Q}_{1}$ and $\mathrm{B}$ due to the shelving are applied. Step (7): Second $1 / 2 \mathrm{CZ}-\pi$ gate between $\mathrm{Q}_{1}$ and B. Step (8): Compensation pulse on $\mathrm{Q}_{2}$. Step (9): Compensation pulse on $\mathrm{Q}_{1}$. Step (10): Compensation delay for the dynamic phase on B. Step (11): Zeroing gate applied to $\mathrm{Q}_{2}$. This step is necessary to re-use $\mathrm{Q}_{2}$ as ancilla qubit for controlling $\mathrm{B}$. The zeroing is performed through an iSWAP between $\mathrm{Q}_{2}$ and $\mathrm{Z}_{2}$. Step (12): The state of $\mathrm{B}$ is read out by the zeroed $\mathrm{Q}_{2}$ via an iSWAP. Steps (13) and (14): A second $\hat{R}_{y}^{\pi / 2}$ rotation on $\mathrm{Q}_{2}$ followed by a measurement pulse completes the Ramsey experiment on B. The Ramsey fringe obtained from this sequence is plotted in Fig. 4E of the main text (magenta dots).

\section{Quantum phase tomography}

The most general unitary operation describing a three-qubit controlled-phase quantum gate can be written as

$$
\widetilde{U}^{\phi}=\left(\begin{array}{cccccccc}
e^{\left.i \phi_{\mid g g}\right\rangle} & 0 & 0 & 0 & 0 & 0 & 0 & 0 \\
0 & e^{i \phi_{|\operatorname{gg} 1\rangle}} & 0 & 0 & 0 & 0 & 0 & 0 \\
0 & 0 & e^{i \phi_{|\mathrm{ge} 0\rangle}} & 0 & 0 & 0 & 0 & 0 \\
0 & 0 & 0 & e^{i \phi_{|\mathrm{ge} 1\rangle}} & 0 & 0 & 0 & 0 \\
0 & 0 & 0 & 0 & e^{i \phi_{|\operatorname{eg} 0\rangle}} & 0 & 0 & 0 \\
0 & 0 & 0 & 0 & 0 & e^{i \phi_{|\lg 1\rangle}} & 0 & 0 \\
0 & 0 & 0 & 0 & 0 & 0 & e^{i \phi_{|\mathrm{ee} 0\rangle}} & 0 \\
0 & 0 & 0 & 0 & 0 & 0 & 0 & e^{i \phi_{|\mathrm{ec} 1\rangle}}
\end{array}\right) .
$$

In the ideal case, the amplitude of each diagonal element of Eq. S26 is unity, while the phase $\phi_{|l m n\rangle}$ depends on which state $|l m n\rangle \in \mathcal{M}_{3}$ is considered (cf. main text). All off-diagonal elements are zero.

It is straightforward to show that only seven of the eight phases of Eq. $\mathrm{S} 26$ are physically independent. In fact, the first complex exponential $e^{i \phi_{|\mathrm{gg} 0\rangle}}$ can be factored out from the equation, allowing us to write the matrix

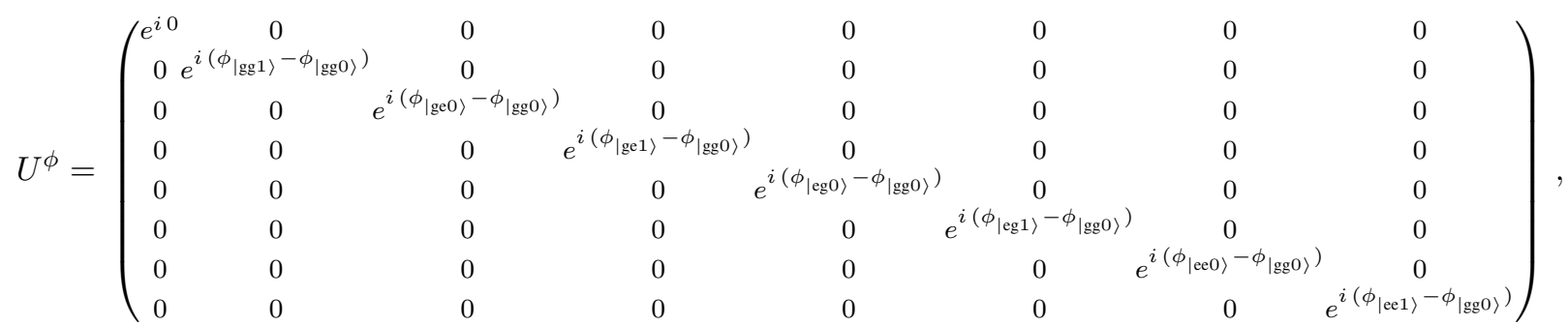

which is equivalent to $\widetilde{U}^{\phi}$ up to a global phase $\phi_{|\operatorname{gg} 0\rangle}$. 
The phases associated with each diagonal element in Eq. S27 can be grouped in a column vector $\tau$ defined as

$$
\tau=\left(\begin{array}{c}
0 \\
\phi_{|\operatorname{gg} 1\rangle}-\phi_{|\operatorname{gg} 0\rangle} \\
\phi_{|\mathrm{ge} 0\rangle}-\phi_{|\operatorname{gg} 0\rangle} \\
\phi_{|\mathrm{ge} 1\rangle}-\phi_{|\operatorname{gg} 0\rangle} \\
\phi_{|\mathrm{eg} 0\rangle}-\phi_{|\operatorname{gg} 0\rangle} \\
\phi_{|\mathrm{eg} 1\rangle}-\phi_{|\operatorname{gg} 0\rangle} \\
\phi_{|\mathrm{ee} 0\rangle}-\phi_{|\operatorname{gg} 0\rangle} \\
\phi_{|\mathrm{ee} 1\rangle}-\phi_{|\operatorname{gg} 0\rangle}
\end{array}\right),
$$

with dimensions $(8,1)$.

In the main text we have shown that by performing Ramsey experiments on the control qubits $\mathrm{Q}_{1}$ and $\mathrm{Q}_{2}$ and on the target resonator $\mathrm{B}$, it is possible to obtain the quantum phase tomography of the three-qubit XOR phase gate and of the Toffoli-class OR phase gate (M gate). In each Ramsey experiment one of the control qubits (or the target resonator) has to be prepared in a $|\mathrm{g}\rangle+|\mathrm{e}\rangle($ or $|0\rangle+|1\rangle)$ state, while the other control qubit and the target resonator (or the two control qubits) are prepared in all four possible combinations of ground and excited state. In the case of an M gate, for example, the twelve states for each Ramsey experiment are reported in the first three columns of Table S3. The fourth column shows the ideal value of the phase difference associated with each Ramsey experiment 17 . A similar Table can easily be obtained for the XOR gate (not shown).

We note that the states of the control qubits $Q_{1}$ and $Q_{2}$ and of the target resonator $B$ displayed in the first three columns of Table $\mathrm{S} 3$ constitute a general set of states for quantum phase tomography and, thus, can be used to characterize any type of three-qubit controlled-phase quantum gate. The phase differences associated with these

Table S3: M gate Ramsey table. The first three columns indicate the state of the control qubits $\mathrm{Q}_{1}$ and $\mathrm{Q}_{2}$ and the state of the target resonator B for the twelve Ramsey experiments needed for quantum phase tomography. The fourth column shows the phase difference obtained from each Ramsey measurement for an ideal M gate.

\begin{tabular}{c|c|c|c}
\hline \hline $\mathrm{Q}_{1}$ & $\mathrm{Q}_{2}$ & $\mathrm{~B}$ & phase difference (rad) - ideal case \\
\hline$|\mathrm{g}\rangle+|\mathrm{e}\rangle$ & $|\mathrm{g}\rangle$ & $|0\rangle$ & 0 \\
\hline$|\mathrm{g}\rangle+|\mathrm{e}\rangle$ & $|\mathrm{g}\rangle$ & $|1\rangle$ & $\pi$ \\
\hline$|\mathrm{g}\rangle+|\mathrm{e}\rangle$ & $|\mathrm{e}\rangle$ & $|0\rangle$ & 0 \\
\hline$|\mathrm{g}\rangle+|\mathrm{e}\rangle$ & $|\mathrm{e}\rangle$ & $|1\rangle$ & 0 \\
\hline$|\mathrm{g}\rangle$ & $|\mathrm{g}\rangle+|\mathrm{e}\rangle$ & $|0\rangle$ & $\pi$ \\
\hline$|\mathrm{g}\rangle$ & $|\mathrm{g}\rangle+|\mathrm{e}\rangle$ & $|1\rangle$ & 0 \\
\hline$|\mathrm{e}\rangle$ & $|\mathrm{g}\rangle+|\mathrm{e}\rangle$ & $|0\rangle$ & 0 \\
\hline$|\mathrm{e}\rangle$ & $|\mathrm{g}\rangle+|\mathrm{e}\rangle$ & $|1\rangle$ & 0 \\
\hline$|\mathrm{g}\rangle$ & $|\mathrm{g}\rangle$ & $|0\rangle+|1\rangle$ & $\pi$ \\
\hline$|\mathrm{g}\rangle$ & $|\mathrm{e}\rangle$ & $|0\rangle+|1\rangle$ & $\pi$ \\
\hline$|\mathrm{e}\rangle$ & $|\mathrm{g}\rangle$ & $|0\rangle+|1\rangle$ & $\pi$ \\
\hline$|\mathrm{e}\rangle$ & $|\mathrm{e}\rangle$ & $|0\rangle+|1\rangle$ & \\
\hline \hline
\end{tabular}


states can be grouped in a column vector $\varphi$ defined as

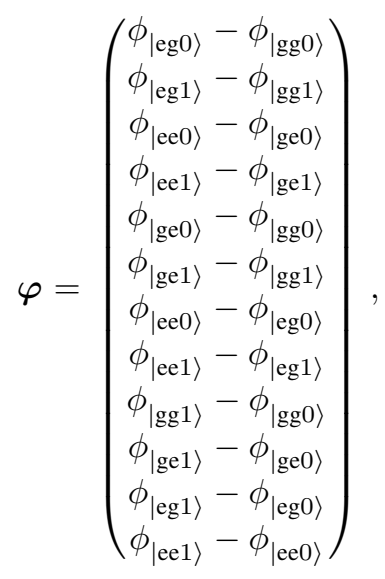

with dimensions $(12,1)$.

The aim of quantum phase tomography is to obtain the seven phase differences in vector $\tau$ from the twelve phase differences in vector $\varphi$, which are measured by means of Ramsey experiments. In order to facilitate the explanation of quantum phase tomography, we now introduce a geometric representation of the phases associated with any three-qubit controlled-phase quantum gate. Figure S11A shows a cube, hereafter termed the phasegate cube, the vertices of which contain information on the diagonal elements of a three-qubit controlled-phase quantum gate. The vertices of the phase-gate cube are enumerated according to the notation given in the space between Fig. 4C and Fig. 4F of the main text. Note that the phase-gate cube can directly be generalized to $N$-qubit gates, in which case it has to be promoted to an $N$-dimensional hypercube.

Here, we will only consider the case of three-qubit gates, namely the XOR gate and $\mathrm{M}$ gate. The quantum logic circuits of these gates are shown in Fig. 4A and Fig. 4D of the main text, respectively. For convenience, these circuits are also displayed in Fig. S11, B and D. The sign of each element $\tau_{k}^{\text {XOR }}$, with $k \in\{0,1, \ldots, 7\}$, of vector $\tau^{\mathrm{XOR}}$ and of each element $\tau_{k}^{\mathrm{M}}$ of vector $\tau^{\mathrm{M}}$ (cf. main text) is given on the vertices of the phase-gate cube (cf. Fig. S11. C and E, respectively). As explained in the main text, a positive sign corresponds to a 0 phase and a negative sign to a $\pi$ phase. As shown in Fig. S11, C and E, the difference between the phases associated with each pair of vertices connected by a segment of the cube is indicated on the segment connecting that pair of vertices. For each gate, this gives a total of twelve phase differences corresponding to the elements in vector $\varphi$ of Eq. S29.

We now use the phase-gate cube to determine the transformation matrix $T_{\varphi \tau}$ between vector $\varphi$ and vector $\tau$. Each row of $T_{\varphi \tau}$ must correspond to a segment of the phase-gate cube, and each column to a vertex, giving a matrix with dimensions $(12,8)$. The first row of $T_{\varphi \tau}$ is associated with the phase difference between states $|\operatorname{eg} 0\rangle$ and $|\operatorname{gg} 0\rangle, \phi_{|\operatorname{eg} 0\rangle}-\phi_{|\operatorname{gg} 0\rangle}$ (cf. Eq. $\mathrm{S} 29$ and, for the case of the M gate, Table S3. Adopting the enumeration in Fig. $\mathrm{S} 11 \mathrm{~A}$, state $|\mathrm{eg} 0\rangle$ corresponds to the vertex (4) of the phase-gate cube, and state $|\operatorname{gg} 0\rangle$ to the vertex (0). In the case of the $\mathrm{M}$ gate, for example, the first raw of $T_{\varphi \tau}$ must then be $-1,0,0,0,1,0,0,0$. Following a similar procedure for all twelve segments of the phase-gate cube for the $\mathrm{M}$ gate, we readily find the entire $\mathrm{M}$ gate 
transformation matrix $T_{\varphi \tau}$

$$
T_{\varphi \tau}=\left(\begin{array}{cccccccc}
-1 & 0 & 0 & 0 & 1 & 0 & 0 & 0 \\
0 & -1 & 0 & 0 & 0 & 1 & 0 & 0 \\
0 & 0 & -1 & 0 & 0 & 0 & 1 & 0 \\
0 & 0 & 0 & -1 & 0 & 0 & 0 & 1 \\
-1 & 0 & 1 & 0 & 0 & 0 & 0 & 0 \\
0 & -1 & 0 & 1 & 0 & 0 & 0 & 0 \\
0 & 0 & 0 & 0 & -1 & 0 & 1 & 0 \\
0 & 0 & 0 & 0 & 0 & -1 & 0 & 1 \\
-1 & 1 & 0 & 0 & 0 & 0 & 0 & 0 \\
0 & 0 & -1 & 1 & 0 & 0 & 0 & 0 \\
0 & 0 & 0 & 0 & -1 & 1 & 0 & 0 \\
0 & 0 & 0 & 0 & 0 & 0 & -1 & 1
\end{array}\right)
$$

Notably, the rank of the matrix $T_{\varphi \tau}$ of Eq. $\mathrm{S} 30$ is 7 , as expected from the number of physically independent phases of the unitary matrix of a general three-qubit controlled-phase quantum gate, $U^{\phi}$. A similar procedure can be used to obtain the transformation matrix associated with the XOR gate (not shown) or any other three-qubit controlled-phase quantum gate.

Given the shape of vectors $\tau$ and $\varphi$, and of the matrix $T_{\varphi \tau}$, vectors $\tau$ and $\varphi$ are related by the simple linear system

$$
T_{\varphi \tau} \cdot \tau=\varphi
$$

Since the phase differences in $\varphi$ are the only phases measured in the experiments, the system of Eq. S31 has to be solved in order to find $\tau$. The matrix $T_{\varphi \tau}$ is actually not invertible. However, the system of Eq. S31 is overconstrained by the experimental data and so it can be solved in a least-squares best fit sense, allowing us to obtain $\tau$.

Figure S12A shows the twelve Ramsey fringes used to measure the phase differences plotted in Fig. S12 C in the case of the XOR gate. Figure $\mathrm{S12}, \mathrm{B}$ and D, shows similar results for the M gate. The phase differences associated with each Ramsey fringe are indicated in the space between panels A and B and, together with the corresponding pair of vertices of the phase-gate cube, in the space between panels $\mathrm{C}$ and $\mathrm{D}$. Solving the system of Eq. S31 for the phase differences shown in Fig. S12, C and D, finally allows us to obtain the phases shown in Fig. 4, C and F, of the main text, thus realizing a full quantum phase tomography of the XOR and M gate.

We note that the quantum phase tomography used here is inherently different from that developed in Ref. $\frac{18}{\text {, }}$ where the time evolution of the quantum phase of the qubit state was used to infer information on the qubit dephasing mechanisms. 
A

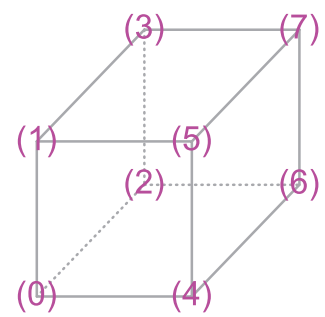

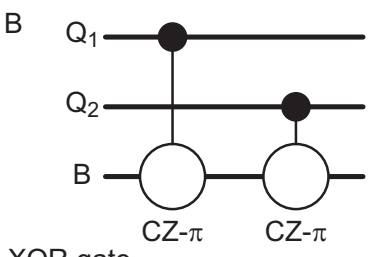

XOR gate

C

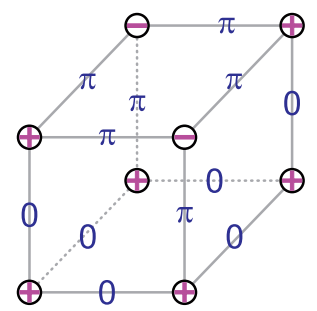

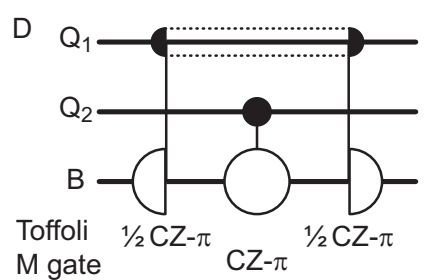

$\mathrm{E}$

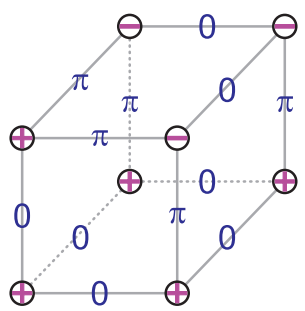

Figure S11: Geometric representation of the phases associated with the three-qubit XOR phase gate and the three-qubit Toffoli-class M gate. (A) The phase-gate cube. The eight vertices of the cube correspond to the diagonal elements of the gate unitary matrix. The vertices are numbered from (0) to (7), following the same enumeration as for the results of quantum phase tomography (cf. space between Fig. 4C and Fig. 4F in the main text). (B) Quantum logic circuit for the XOR gate, as in Fig. 4A of the main text. (C) The sign of each element $\tau_{k}^{\mathrm{XOR}}$ of vector $\tau^{\mathrm{XOR}}$ (cf. main text) is given on the vertices of the phase-gate cube. Each sign, +1 or -1 , corresponds to a phase, 0 or $\pi$ (cf. main text). The difference between the phases associated with each pair of vertices connected by a segment of the cube (a total of twelve phase differences) is indicated on the segment connecting that pair of vertices. (D) Quantum logic circuit for the M gate, as in Fig. 4D of the main text. (E) As in C, but for the M gate. 


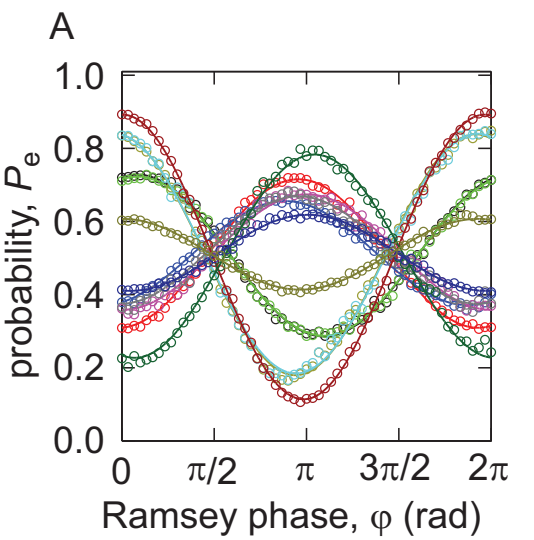

C

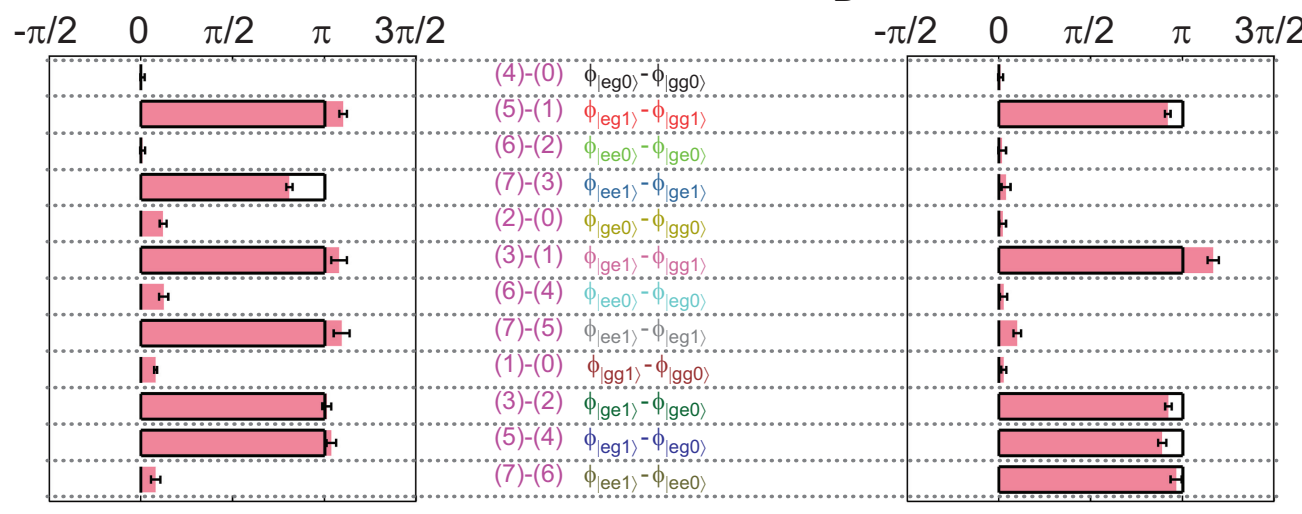

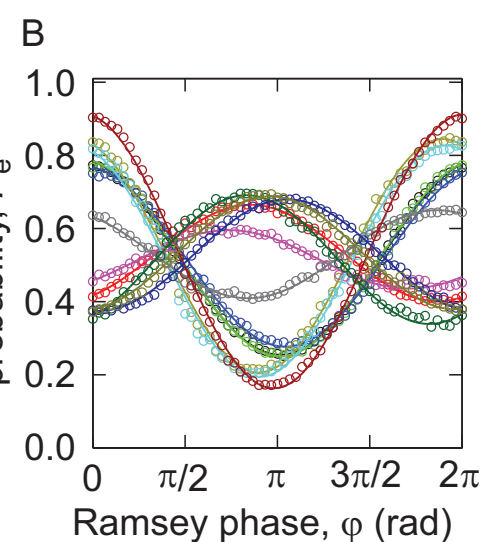

$\stackrel{\circ}{\circ} \phi_{\mid \text {eg } 0\rangle}-\phi_{|g g 0\rangle}$

$\stackrel{\circ}{\circ 0} \phi_{|\operatorname{eg} 1\rangle}{ }^{-} \phi_{\mid g g 1}$

$\stackrel{\circ \circ 0}{\circ} \phi_{(e e 0)}-\phi_{\mid g e 0}$

$\stackrel{0}{\circ} \phi_{\mid \text {ee } 1\rangle}-\phi_{|g e 1\rangle}$

$\stackrel{0}{\circ} \phi_{|\mathrm{ge} 0\rangle}-\phi_{|\mathrm{gg} 0\rangle}$

$\stackrel{0}{0} \phi_{|g e 1\rangle}-\phi_{|g g 1\rangle}$

$\phi_{\text {ee } 0\rangle} \phi \phi_{\text {eg } 0}$

$0_{0}^{\circ} \phi_{|e e 1\rangle}-\phi_{|\operatorname{eg} 1\rangle}$

$\stackrel{\circ}{\circ} \phi_{|g g 1\rangle}-\phi_{|g g 0\rangle}$

$\stackrel{\circ}{\circ} \phi_{|g e 1\rangle} \phi_{\mid g e 0}$

응 $\phi_{|\operatorname{eg} 1\rangle}-\phi_{|\operatorname{eg} 0\rangle}$

$\stackrel{\circ}{\circ 0} \phi_{\mid \text {ee } 1\rangle}-\phi_{\mid \text {ee } 0\rangle}$

Ramsey phase, $\varphi(\mathrm{rad})$

$\mathrm{D}$

$\pi / 2$

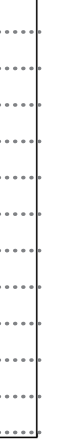

Figure S12: Quantum phase tomography for the XOR and M gate. (A) Probability $P_{\mathrm{e}}$ to measure either of the control qubits $\mathrm{Q}_{1}$ or $\mathrm{Q}_{2}$ or target B in state $|\mathrm{e}\rangle$ or $|1\rangle$ vs. Ramsey phase $\varphi$ for the XOR gate. (B) As in A, but for the M gate. Open circles: Data. Solid lines: Least-squares fits to the data. The legend to the phases is indicated in the space between the panels. (C) The twelve phase differences in vector $\varphi$ obtained from the Ramsey fringes in A. (D) As in C, but for the phase differences obtained from the Ramsey fringes in B. The pair of vertices of the phase-gate cube and the corresponding phase differences are indicated in the space between the panels. The error bars are due to the confidence intervals to the fits in A and B. Such confidence intervals propagate through the quantum phase tomography process generating the error bars in Fig. 4, C and F, of the main text. 


\section{References}

1. M. Steffen, M. Ansmann, R. McDermott, N. Katz, R. C. Bialczak, E. Lucero, M. Neeley, E. M. Weig, A. N. Cleland \& J. M. Martinis, State tomography of capacitively shunted phase qubits with high fidelity. Phys. Rev. Lett. 97, 050502 (2006).

2. M. A. Nielsen \& I. L. Chuang, Quantum computation and quantum information. (Cambridge Univ. Press, Cambridge - UK, 2000).

3. A. G. Kofman \& A. N. Korotkov, Two-qubit decoherence mechanisms revealed via quantum process tomography. Phys. Rev. A 80, 042103 (2009).

4. R. C. Bialczak, M. Ansmann, M. Hofheinz, E. Lucero, M. Neeley, A. D. O’Connell, D. Sank, H. Wang, J. Wenner, M. Steffen, A. N. Cleland \& J. M. Martinis, Quantum process tomography of a universal entangling gate implemented with Josephson phase qubits. Nature Phys. 6, 409-413 (2010).

5. T. Yamamoto, M. Neeley, E. Lucero, R. C. Bialczak, J. Kelly, M. Lenander, M. Mariantoni, A. D. O'Connell, D. Sank, H. Wang, M. Weides, J. Wenner, Y. Yin, A. N. Cleland \& J. M. Martinis, Quantum process tomography of two-qubit controlled-Z and controlled-NOT gates using superconducting phase qubits. Phys. Rev. $B$ 82, 184515 (2010).

6. R. Horodecki, P. Horodecki, M. Horodecki \& K. Horodecki, Quantum entanglement. Rev. Mod. Phys. 81, 865-942 (2009).

7. M. Mariantoni, H. Wang, R. C. Bialczak, M. Lenander, E. Lucero, M. Neeley, A. D. O'Connell, D. Sank, M. Weides, J. Wenner, T. Yamamoto, Y. Yin, J. Zhao, J. M. Martinis \& A. N. Cleland, Photon shell game in three-resonator circuit quantum electrodynamics. Nature Phys. 7, 287-293 (2011).

8. M. Steffen, M. Ansmann, R. C. Bialczak, N. Katz, E. Lucero, R. McDermott, M. Neeley, E. M. Weig, A. N. Cleland \& J. M. Martinis, Measurement of the entanglement of two superconducting qubits via state tomography. Science 313, 1423-1425 (2006).

9. The MATLAB packages SeDuMi 1.21 and YALMIP can be downloaded freely at http://sedumi.ie.lehigh.edu/ and http://users.isy.liu.se/johanl/yalmip/, respectively.

10. Note that, in general, the two-level approximation is unsuitable for describing a phase qubit. In fact, the phase qubit nonlinearity is typically small and, thus, at least the three lowest eigenstates $|\mathrm{g}\rangle,|\mathrm{e}\rangle$, and $|\mathrm{f}\rangle$ should be taken into account for a more correct description. However, for the purposes of this section we will only consider states $|\mathrm{g}\rangle$ and $|\mathrm{e}\rangle$.

11. A more complete description of the qubit-driving Hamiltonian should also include a term $\approx$ $h \gamma \Omega_{\mathrm{D}}(\tau) \hat{\sigma}_{z} \sin \left(2 \pi f_{\mathrm{D}} \tau+\phi_{\text {del }}\right)$, where $\gamma$ is a small parameter $(\gamma \simeq 0)$. This term slightly shifts the qubit eigenenergies and, for a small-amplitude driving (as in our case), it can safely be neglected.

12. In reality, we use a single carrier signal with frequency usually different from $f_{\mathrm{Q}}^{0}$. The qubit can be excited resonantly by means of side-band mixing, which gives us more flexibility during the experiments ${ }^{1}$.

13. C. Cohen-Tannoudji, B. Diu \& F. Laloë, Quantum mechanics - Vol. I. (John Wiley \& Sons, Inc., New York USA, 1977).

14. J. J. Sakurai, Modern quantum mechanics - revised ed.. (Addison-Wesley Publ. Comp., Reading - USA, 1994).

15. H. Wang, M. Hofheinz, M. Ansmann, R. C. Bialczak, E. Lucero, M. Neeley, A. D. O'Connell, D. Sank, J. Wenner, A. N. Cleland \& J. M. Martinis, Measurement of the decay of Fock states in a superconducting quantum circuit. Phys. Rev. Lett. 101, 240401 (2008). 
16. H. Wang, M. Hofheinz, M. Ansmann, R. C. Bialczak, E. Lucero, M. Neeley, A. D. O'Connell, D. Sank, M. Weides, J. Wenner, A. N. Cleland \& J. M. Martinis, Decoherence dynamics of complex photon states in a superconducting circuit. Phys. Rev. Lett. 103, 200404 (2009).

17. In the experiments, the phase difference associated with the states in the first row of Table $\mathrm{S3}$ is used as a reference phase for each phase difference associated with the states in the remaining rows. A similar approach is followed for the XOR gate.

18. M. S. Rudner, A. V. Shytov, L. S. Levitov, D. M. Berns, W. D. Oliver, S. O. Valenzuela \& T. P. Orlando, Quantum phase tomography of a strongly driven qubit. Phys. Rev. Lett. 101, 190502 (2008). 